\title{
Mean-field view on geodynamo models
}

\author{
Dissertation \\ zur Erlangung des Doktorgrades \\ der Mathematisch-Naturwissenschaftlichen Fakultäten \\ der Georg-August-Universität zu Göttingen
}

vorgelegt von

Martin Schrinner

aus Eschwege

Göttingen 2005 
D7

Referent: Prof. Dr. A. Tilgner

Korreferent: Prof. Dr. U. Christensen

Tag der mündlichen Prüfung: 13.7.2005 


\section{Contents}

$\begin{array}{ll}\text { Summary } & 5\end{array}$

$\begin{array}{llr}1 & \text { Introduction } & 7\end{array}$

2 Numerical modelling of the geodynamo $\quad 13$

2.1 Model equations . . . . . . . . . . . . . . . . . . . . . . . . 13

2.2 Numerical techniques . . . . . . . . . . . . . . . . . . . . . 14

3 Mean-field theory $\quad 17$

3.1 The mean-field concept . . . . . . . . . . . . . . . . . . . . . 17

3.2 Mean-field coefficients in spherical geometry . . . . . . . . . . . . . 19

3.3 The second order correlation approximation . . . . . . . . . 22

3.4 Symmetry properties of mean-field coefficients . . . . . . . . . . 23

4 How to derive mean-field coefficients $\quad 27$

4.1 Approach (I) . . . . . . . . . . . . . . . . . 27

4.1 .1 The method . . . . . . . . . . . . . . 27

4.1 .2 The choice of test fields . . . . . . . . . . . . . . 29

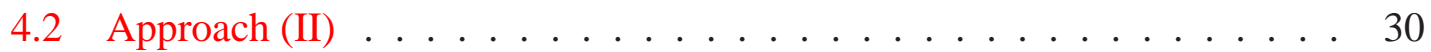

5 Mean-field coefficients: results $\quad 35$

5.1 Simulation of rotating magnetoconvection . . . . . . . . . . . 35

5.1 .1 Velocity and magnetic field . . . . . . . . . . . 35

5.1.2 Non-covariant and covariant mean-field coefficients . . . . . . . . 37

5.1 .3 Isotropic approximation . . . . . . . . . . . . . . 42

$5.1 .4 \alpha$ - and $\beta$-quenching .......................... 42

5.2 A simple quasi-stationary dynamo . . . . . . . . . . . . . . . . 47

5.2.1 Characteristics of the dynamo and supplements to approach (I) . . 47

5.2 .2 Mean-field coefficients . . . . . . . . . . . . . . 48

5.2.3 Beyond $\tilde{\boldsymbol{a}}$ and $\tilde{\boldsymbol{b}}$ : the expansion of $\mathcal{E}$ including derivatives of $\overline{\boldsymbol{B}}$ up to the second order . . . . . . . . . . . . 48

5.2 .4 Shortcomings due to SOCA . . . . . . . . . . . . 50 50

5.3 A highly time-dependent dynamo in the strongly columnar regime . . . . 51

5.3.1 Characteristics of the dynamo and mean-field coefficients . . . . 51

5.3.2 Time-variability of mean-field coefficients . . . . . . . . . . 52

5.4 A highly time-dependent dynamo in the fully developed regime . . . . . . 55 
5.4.1 Characteristics of the dynamo, adaption of approach (I), and resulting mean-field coefficients . . . . . . . . . . . . 55

5.4.2 Time variability of mean-field coefficients and reversals . . . . 59

6 Two-dimensional mean-field model $\quad 63$

6.1 Model equations . . . . . . . . . . . . . . . . . . . . . . 63

6.2 Numerical techniques . . . . . . . . . . . . . . . . . . 65

6.3 Free-decay mode test . . . . . . . . . . . . . . . . 66

7 Comparison between direct numerical simulations and mean-field calcula$\begin{array}{ll}\text { tions } & 69\end{array}$

7.1 Success of mean-field models and their limits . . . . . . . . . . . . 69

7.2 Action and significance of mean-field coefficients . . . . . . . . . . 75

8 Conclusions and outlook $\quad 79$

A Relations between covariant and non-covariant mean-field coefficients $\quad \mathbf{8 3}$

B Representation of a second rank tensor which depends on two directions $\quad \mathbf{8 5}$

$\begin{array}{lll}\text { C Green's functions and orthogonality relations } & 87\end{array}$

D Components of $\tilde{a}$ in SOCA

E Comparison between approach (I) and approach (II) 95

F Mean-field coefficients for the example of the benchmark dynamo 97

$\begin{array}{ll}\text { Bibliography } & 99\end{array}$

$\begin{array}{ll}\text { Scientific contributions } & 105\end{array}$

$\begin{array}{ll}\text { Danksagung } & 105\end{array}$

$\begin{array}{ll}\text { Lebenslauf } & 107\end{array}$ 


\section{Summary}

Mean-field theory provides a useful description of magnetohydrodynamic processes leading to large-scale magnetic fields in various cosmic objects. In this study, dynamo processes in a rotating spherical shell have been considered, and mean fields have been defined by azimuthal averaging. In mean-field theory, the coefficients occurring in the expansion of the mean electromotive force in terms of the mean field and its derivatives are used to analyse and to simulate dynamo action. In this work, dynamo processes present in geodynamo simulations have been studied by computing corresponding mean-field coefficients. Furthermore, their dynamo action in a mean-field simulation has been examined. For this purpose, two methods to determine mean-field coefficients have been developed:

Approach (I) is based on the numerical computation of electromotive forces for a number of imposed mean test fields. This requires one to solve the induction equation for the non-axisymmetric, residual field numerically. Subsequently, the linear relation between the mean electromotive forces and the test-fields is inverted to solve for the meanfield coefficients.

Approach (II) aims at deriving quasi-analytical expressions for the mean electromotive force and finally for the mean-field coefficients. Again, the residual magnetic field for a given velocity field has to be known. Applying the second order correlation approximation, assuming stationarity, and neglecting the mean flow, the induction equation for the residual field may be integrated analytically. This has been done by means of a poloidal/toroidal decomposition of the velocity field and the magnetic field. A subsequent expansion in spherical harmonics converts the angular derivatives to algebraic relations. The remaining integration over the radial coordinate has been carried out with the help of appropriate Green's functions.

Both methods have been applied to a simulation of rotating magnetoconvection and a simple quasi-stationary dynamo (hereafter referred to as benchmark example). They are consistent with each other in a parameter regime in which the second order correlation approximation (SOCA) is justified. In general, however, mean-field coefficients determined by means of SOCA exhibit overestimated amplitudes.

In both examples, the resulting tensorial mean-field coefficients are highly anisotropic and demonstrate the existence of an $\alpha^{2}$-mechanism along with a strong $\gamma$-effect operating outside the inner core tangent cylinder. The turbulent diffusivity exceeds the molecular one by at least one order of magnitude in the benchmark example. However, the turbulent diffusion may also be moderated by a $\delta \times j$-effect due to a turbulent conductivity with a conductivity tensor which is no longer symmetric.

Moreover, the quenching of relevant mean-field coefficients, e.g. the $\alpha$ - and $\beta$-quenching, resulting from the back reaction of the Lorentz force on the velocity field has been examined in the magnetoconvection example. Both the $\alpha$ - and the $\beta$-components are 
quenched to low values if the strength of the mean magnetic field exceeds the equipartition field strength by at least a factor of five.

Approach (I) has likewise been applied to two highly time-dependent dynamos, one in the strongly columnar and the other in the fully developed regime. The resulting timeaveraged mean-field coefficients resemble those obtained in the magnetoconvection and benchmark example, which indicates that similar dynamo processes take place.

The temporal fluctuations of mean-field coefficients occur on timescales of the convective turnover time. They exhibit particularly large amplitudes for the dynamo in the fully developed regime, in which the velocity field lacks any equatorial symmetry.

With the aim of comparing mean-field simulations with corresponding direct numerical simulations, a two-dimensional mean-field model involving all previously determined mean-field coefficients has been constructed. Various tests with different sets of meanfield coefficients reveal their action and significance. In the magnetoconvection and benchmark example considered here, the match between direct numerical simulations and mean-field simulations is best if at least 17 mean-field coefficients are kept. In the magnetoconvection example, the azimuthally averaged magnetic field resulting from a direct numerical simulation is in good agreement with a corresponding result given by the mean-field model. However, this match is not satisfactory in the benchmark example. Here, the traditional representation of the mean electromotive force including no higher than first-order derivatives is no longer justified. The lack of a clear scale separation renders the applicability of the traditional mean-field approach inappropriate in this example. 


\section{Introduction}

"How could a rotating body such as the Sun become a magnet?", asked Sir Joseph Larmor in a famous article in 1919 (Larmor 1919). While the origin of the magnetic field of the Sun was at that time a total mystery, the magnetic field of the Earth did not excite similar inquiry because it was still believed that the Earth's magnetic field could be explained in terms of permanent magnetisation (Moffatt 1978). However, today it seems to be evident that large-scale magnetic fields as the Earth's as well as the solar or the galactic magnetic field are maintained by hydromagnetic dynamos (Weiss 2002). In the case of the Earth, the timescale of ohmic decay in the Earth's core is of several thousand years, whereas the field has been present for at least 3.5 Gyrs. In addition, it is now well known that the temperature of the Earth's interior is above the Curie temperature at which ferromagnetic materials loose their permanent magnetisation. Further observations which contradict the hypothesis of permanent magnetisation are the secular variation of the Earth's magnetic field and polarity reversals which occurred in the Earth's history as proven by paleomagnetic records (Fearn 1998, Roberts and Glatzmaier 2000).

Although Faraday had demonstrated that currents can be driven by the inductive effect of a disc rotating in the field of a permanent magnet and Siemens had succeeded in constructing a self-exciting dynamo in 1866, Larmor's proposal (1919) that "it is possible for the [Sun's] internal cyclic motion to act after the manner of the cycle of a selfexciting dynamo" was controversial. In particular, Cowling (1933), who had shown that an axisymmetric magnetic field could not be maintained by hydromagnetic dynamo action, objected: "The theory proposed by Sir Joseph Larmor, that the magnetic field of a sunspot is maintained by the currents it induces in moving matter, is examined and shown to be faulty: [...]". The initial controversy about the existence of self-excited dynamos driven by fluid motions originated from the complexity of the dynamo problem: It is intrinsically three-dimensional and nonlinear due to the back-reaction of the Lorentz force. Meanwhile, the existence of dynamo action by fluid flow had been proven theoretically and was demonstrated in the recent past through the laboratory dynamo experiments in Riga and Karlsruhe (Müller and Stieglitz 2000, Gailitis et al. 2000).

Nevertheless, global computational dynamo models simulate only the geodynamo reasonably well, while it remains difficult to tackle the stellar or galactic dynamo problem. Despite the increasing computational power, a direct numerical treatment of the governing partial differential equations is not yet feasible in the latter cases, because of the huge range of spatial and temporal scales needing to be resolved there (Tobias 2002, Weiss and Tobias 2000, Shukurov 2002). First attempts of global MHD simulations have been carried out by Gilman (Gilman and Miller 1981, Gilman 1983) adopting the Boussinesq approximation and later by Glatzmaier (Glatzmaier 1984, 1985) using an anelastic model. Both aimed to model the solar dynamo. Though cyclic dynamo solutions were obtained 
in some cases, the models exhibited a wrong poleward migration of the magnetic field (Glatzmaier 1985). In a recent attempt, Brun et al. (2004) succeeded in simulating a solar-like differential rotation but can not reproduce any features of the solar cycle yet.

An alternative approach is provided by mean-field electrodynamics (Steenbeck et al. 1966, Moffatt 1978, Krause and Rädler 1980), which is a statistical theory focussing only on large scale, i.e. averaged fields. Highly complex small-scale or residual parts need not to be known in detail, only the averaged cross product of the residual velocity and magnetic field, in the following called the mean electromotive force, is relevant and accounts for the evolution of the mean field. Advantageously, the difficulties in resolving the small-scale structures can be avoided. Usually, the action of the small-scale velocity on the mean magnetic field as expressed by the mean electromotive force, $\mathcal{E}$, is parametrised, and the parameters are known as mean-field coefficients. Most prominent among them is the so-called $\alpha$-tensor. It is closely related to a fundamental induction effect associated with cyclonic convection (Parker 1955, 1957) which is nowadays known as $\alpha$-effect for this reason. Other mean-field coefficients contribute to the advection and diffusion of the mean magnetic-field. In other words, mean-field theory supplies theoretical insight as well as formalised physical concepts in order to interpret and, in principle, also to quantify dynamo action.

Despite their relative simplicity, mean-field models have reproduced basic features of the solar cycle successfully (see e.g. Stix 2002, Ossendrijver 2003) and are moreover unique in simulating many features of the magnetic field in spiral galaxies coherently (Beck et al. 1996, Shukurov 2002). But, whether mean-field models show dynamo action or not depends strongly on the set of chosen mean-field coefficients, which are in general not known but determined in a reasonable, but nevertheless arbitrary way. Thus, meanfield models are subject to the fundamental criticism that they just reproduce what was stuck into them beforehand.

Concerning the geodynamo, the situation is very much different. Many features of the Earth's magnetic field have been successfully reproduced by nonlinear three-dimensional simulations of the magnetohydrodynamics in the Earth's core. Although some model parameters still do not reach realistic values, and in particular viscous effects and therefore the size of viscous boundary layers are by orders of magnitude overestimated, the simulations exhibit an axial dipole dominated magnetic field at the Earth's surface that is maintained over several magnetic diffusion times (Glatzmaier and Roberts 1995b, Kuang and Bloxham 1997, Christensen et al. 1998). In addition, the time-dependence of the dipole moment, including secular variation, excursions and reversals, resembles the observed Earth's magnetic field (Glatzmaier and Roberts 1995a, Kutzner and Christensen 2002). The success in simulating the geodynamo can be attributed to the rather moderate vigour of turbulence in the Earth's outer core compared to the much more turbulent dynamics in the solar convection zone. The difference is formally expressed in terms of very different magnetic Reynolds numbers: While Fearn (1998) estimates $R m \sim O\left(10^{3}\right)$ for the Earth's outer core a representative value of $R m$ at the base of the solar convection zone is $R m \approx 10^{10}$ (Ossendrijver 2003).

Even though present geodynamo models are fully self-consistent their interpretation relies frequently in a heuristic manner on mean-field concepts (Glatzmaier and Roberts 1995b, Kageyama and Sato 1997, Olson et al. 1999). This indicates that the need for fundamental physical concepts explaining dynamo processes makes mean-field theory 
extremely useful and indispensable at the present time. However, the applicability of mean-field concepts as tools for analysing dynamo processes in direct numerical simulations suffers again from the poor knowledge of mean-field coefficients and reliable methods to derive them.

There are two seminal approaches which have been followed in order to determine mean-field coefficients. The first one makes use of numerical modelling, in which the mean electromotive force, $\mathcal{E}$, as well as the mean-field, $\overline{\boldsymbol{B}}$, are determined numerically as an output of an MHD-simulation. Further on, a linear relation between $\mathcal{E}$ and $\overline{\boldsymbol{B}}$ is assumed, which has to be inverted in order to solve for the unknown mean-field coefficients. However, a fundamental problem related to this approach arises since the number of unknown variables is in general much higher than the number of equations resulting from the linear relation between $\mathcal{E}$ and $\overline{\boldsymbol{B}}$. Therefore, all work presented so far refers either to specific situations in which certain constraints reduce the number of mean-field coefficients beforehand, or most of the mean-field coefficients are considered as small and are simply neglected. Ziegler et al. (1996) calculated the $\alpha$-tensor for the galactic dynamo due to numerical simulations of supernova explosions and confirmed the results given by Ferrière (Ferrière 1993a, see below) whereas further terms in the expansion of $\mathcal{E}$ have been neglected. Following also the first approach described above, Ossendrijver et al. $(2001,2002)$ used box simulations of magnetoconvection in the solar convection zone to determine the local $\alpha$-tensor in Cartesian geometry. Both, Ziegler et al. (1996) and Ossendrijver et al. (2002) performed three numerical experiments with imposed mean magnetic field in orthogonal directions and combined the results in order to close the system of linear equations. In a further attempt to determine not only the local $\alpha$-tensor but the $\beta$-tensor as well, Brandenburg and Sokoloff (2002) applied numerical simulations of turbulence in accretion disks. As one result, they obtained a negative coefficient of turbulent diffusion, which was considered as unphysical by the authors. Their idea of how to increase the number of defining linear equations has been to exploit the time dependence of $\mathcal{E}$ and $\overline{\boldsymbol{B}}$, that is, they considered expansions of $\mathcal{E}_{i}$ correlated with components of $\overline{\boldsymbol{B}}$ in order to increase the number of linear equations.

With the aim to study the saturation of the turbulent $\alpha$-effect in the nonlinear regime, Cattaneo and Hughes (1996) determined a box-averaged $\alpha$-scalar, which thus has lost any spatial dependence. Anyhow, their paper attracted interest because they found $\alpha$ remarkebly quenched far below the equipartition value of $\bar{B}$ and postulated therefore a catastrophic $\alpha$-quenching for highly turbulent flows.

The second approach aims at deriving a (quasi-)analytical expression for the mean electromotive force which can be evaluated for mean-field coefficients afterwards (see e.g. Moffatt 1978, Krause and Rädler 1980). This requires one to integrate the governing equation for the residual magnetic field with the help of closure methods. Most commonly used is the second order correlation approximation (SOCA) in which only statistical moments up to the second order are taken into account, while moments of higher order are neglected. Following this approach, an early result for a scalar $\alpha$ is given by Steenbeck and Krause (1969). Further calculations by Kichatinov and Rüdiger (1992), Rüdiger and Kichatinov (1993) provide more $\alpha$-coefficients but rely on specific symmetry assumptions and various additional approximations, valid in the high conductivity limit and for high and low rotation rates, respectively. In the context of the galactic dynamo, Ferrière (1992) used the SOCA-closure and the frozen flux approximation to conclude an analyti- 
cal expression for the mean electromotive force based on turbulence driven by supernova and superbubble explosions. In a series of following papers, Ferrière (1993a,b) gave also formulas for the nonvanishing components of the $\alpha$ - and $\beta$-tensor in cylindrical geometry. By the same token, the dynamo action of idealised, spatially periodic flows, realised in the Karlsruhe dynamo experiment for instance, has been intensively studied with means of the SOCA-closure (Rädler et al. 2002, Tilgner 2004). In these cases, the velocity field is given analytically and possesses a number of simplifying symmetries, which allows a rather direct computation of mean-field coefficients.

Other closure methods, as the 'Eddy-damped quasi-normal Markovian' closure (EDQNM) (Orszag 1970) and the related $\tau$-approximation (Pouquet et al. 1976), have been likewise applied. The quasi-normal approximation replaces fourth-order moments by a sum of products of second-order moments, which is to some extent arbitrary. Nevertheless, this closure scheme seems to be consistent with a general description of turbulence (Moffatt 1978) and is frequently used by, e.g., Kleeorin and Rogachevskii (2003) to investigate the dependency of the $\alpha$-tensor on rotation rate or to study the $\alpha$-effect, turbulent diffusion and the shear-current effect in the nonlinear regime (Rogachevskii and Kleeorin 2000, 2004).

The idea of this thesis work is to take advantage of global, direct numerical simulations as performed to simulate the geodynamo and to compare them with respective mean-field calculations. This will lead to an estimation of the reliability of mean-field theory and its often used approximations. On the other hand, such a comparison will help to improve the conceptual understanding of dynamo mechanisms which are observed in direct numerical simulations.

As already pointed out earlier, both aims are intimately associated with the derivation of corresponding mean-field coefficients. Hence, emphasis is placed on the developement of two methods which contribute to each of the principal approches mentioned above. Both methods confirm each other and serve as powerful tools to determine a number of relevant mean-field coefficients. While most of the quoted earlier work refers to a cartesian-box geometry, global mean-field coefficients for the astrophysically more relevant domain of a rotating sphere are presented here, and specific problems related to the spherical geometry are discussed.

Besides their use in a mean-field model, the resulting mean-field coefficients are of relevance because of their associated physical meaning. Thus, it will be interesting to learn about their spatial and also temporal dependencies. Is it possible to derive some constraints on their amplitude or morphology? How do the actual mean-field coefficients deviate from those derived in the isotropic or second order correlation approximation? To which extend are mean-field coefficients quenched due to nonlinear effects? So as to tackle these questions, a sample of four very different models is examined. The simplest one is not a self-excited dynamo but a simulation of rotating magnetoconvection at a rather moderate magnetic Reynolds number, the most complicated a reversing dynamo in a very different flow regime. These investigations are not meant to be a systematic parameter study but rather a first glimpse to gain insight into how mean-field coefficients vary with the character of the flow.

In order to carry out the intended comparison between mean-field theory and direct numerical simulations, a two-dimensional mean-field model has been constructed, which relies on the set of determined mean-field coefficients. It has been used to test important 
assumptions on which mean-field models are frequently based, e.g. the assumption of a scale separation or the applicability of the second order correlation approximation.

All implemented coefficients in the mean-field model can be weighted giving the possibility to isolate and therefore identify certain dynamo processes. Which of the meanfield coefficients have importance and what is their related action in a mean-field model, which of them are negligible, are further questions which will be addressed within the scope of this work. 



\section{Numerical modelling of the geodynamo}

All self-consistent dynamo calculations presented here have been carried out with a numerical model which was designed in its original form by Glatzmaier (1984). This version of the code solved the anelastic magnetohydrodynamic equations in a spherical shell to simulate stellar dynamos. Olson and Glatzmaier (1995) later applied a modified version of the numerical model to run siumulations of magnetoconvection in a rotating spherical shell adopting the Boussinesq approximation. Further changes by Christensen et al. (1999) result in an explicit treatment of the Coriolis force and in a modified time-step control. Meanwhile, the code has been validated by benchmarking it with other threedimensional models (Christensen et al. 2001). In the following, a short description of the model equations in dimensionless form and the used numerical techniques to solve them is given. For a more detailed discussion I refer to the quoted literature.

\subsection{Model equations}

A spherical shell of electrically conducting fluid is considered in which the fluid velocity $\boldsymbol{V}$, the magnetic field $\boldsymbol{B}$ and the temperature $T$ are governed by

$$
\begin{gathered}
E\left(\frac{\partial \boldsymbol{V}}{\partial t}+\boldsymbol{V} \cdot \nabla \boldsymbol{V}-\nabla^{2} \boldsymbol{V}\right)+2 \hat{\boldsymbol{z}} \times \boldsymbol{V}+\nabla P=R a \frac{\boldsymbol{r}}{r_{o}} T+\frac{1}{P m}(\nabla \times \boldsymbol{B}) \times \boldsymbol{B} \\
\nabla \cdot \boldsymbol{V}=0 \\
\frac{\partial T}{\partial t}+\boldsymbol{V} \cdot \nabla T=\frac{1}{P r} \nabla^{2} T \\
\frac{\partial \boldsymbol{B}}{\partial t}=\nabla \times(\boldsymbol{V} \times \boldsymbol{B})+\frac{1}{P m} \nabla^{2} \boldsymbol{B} \\
\nabla \cdot \boldsymbol{B}=0
\end{gathered}
$$

The fluid dynamic equations have to be understood as Boussinesq approximation. The motion is measured relative to the uniform rotation of the shell with angular velocity $\Omega \hat{z}$ at which $\hat{z}$ is a unit vector in the direction of the rotation axis. The governing dimensionless parameters of the model are the Ekman number $E=\nu / \Omega D^{2}$, the (modified) Rayleigh number $R a=\alpha_{T} g_{0} \Delta T D / \nu \Omega$, the Prandtl number $\operatorname{Pr}=\nu / \kappa$ and the magnetic Prandtl 
number $P m=\nu / \eta$. In these expressions, $\nu$ denotes the kinematic viscosity, $D$ the shell width, $\alpha_{T}$ the thermal expansion coefficient, $g_{0}$ is the gravitational acceleration at the outer boundary $r=r_{o}, \Delta T$ means the temperature difference between the inner and outer spherical boundaries, $\kappa$ is the thermal and $\eta=1 / \mu \sigma$ the magnetic diffusivity with the magnetic permeability $\mu$ and the electrical conductivity $\sigma$. This choice of dimensionless parameters implies that $D, D^{2} / \nu$, and $(\varrho \mu \eta \Omega)^{1 / 2}$, in which $\varrho$ is the density, serve as the length scale, time scale and scale for the magnetic field, respectively. The ratio of outer to inner radius of the shell is $r_{o} / r_{i}=0.35$, and thus $D=0.65 r_{o}$ for all simulations considered here. For the velocity $\boldsymbol{V}$, no-slip boundary conditions are adopted:

$$
\boldsymbol{V}=0 \quad \text { at } r=r_{i}, r=r_{o}
$$

Moreover, all surroundings of the spherical shell are assumed as electrically non-conducting, so the magnetic field $\boldsymbol{B}$ continues as a potential field in both parts exterior to the fluid shell. However, for the simulation of magnetoconvection, an imposed toroidal field is assumed, resulting from electric currents due to sources or sinks on the boundaries, and the magnetic boundary conditions are changed accordingly. The temperature is assumed to be constant on the boundaries, so that

$$
T=1 \quad \text { at } r=r_{i}, \quad T=0 \quad \text { at } r=r_{o}
$$

The initial conditions are not thought to be of essential importance since the simulations usually reach a statistical equilibrium after a transient period in the sense that appropriate time averages of the magnetic or the kinetic energy density, for instance, become steady. For practical reasons it is of course reasonable to start calculations with adjacent solutions in parameter space.

In order to characterise the results of the simulations, the magnetic Reynolds number $R m=v D / \eta$ with $v$ interpreted as r.m.s. velocity and the Elsasser number $\Lambda=B^{2} / \varrho \mu \eta \Omega$ with $B$ interpreted as the r.m.s. value of the magnetic field inside the shell are used.

\subsection{Numerical techniques}

Since velocity and magnetic field are assumed to be solenoidal, both can be represented as a sum of toroidal and poloidal vectors in the form

$$
\begin{gathered}
\boldsymbol{V}=\nabla \times(z \hat{\boldsymbol{r}})+\nabla \times \nabla \times(w \hat{\boldsymbol{r}}) \\
\boldsymbol{B}=\nabla \times(j \hat{\boldsymbol{r}})+\nabla \times \nabla \times(b \hat{\boldsymbol{r}})
\end{gathered}
$$

with the defining scalars $z, w$ and $j, b$, respectively; $\hat{\boldsymbol{r}}$ denotes a unit vector in radial direction. Thus, together with the temperature, there are five variables which are determined by solving the radial component of the momentum equation (2.1), the radial component of the curl of the momentum equation, the radial component of the induction equation (2.2), the radial component of the curl of the induction equation, and the temperature equation (2.3). A complementing equation to determine the pressure is given by taking the divergence of the momentum equation. All variables are expanded in spherical harmonics to 
resolve their horizontal structure and in Chebyshev polynomials to resolve their radial dependence. While spatial derivatives are most conveniently taken in spectral space, all nonlinear products are calculated in grid space. The discretisation in time is performed by an implicit Crank-Nicholson scheme and by an explicit Adams-Bashforth scheme for the treatment of the nonlinear terms and the Coriolis force. The resulting equations are finally solved by a Chebyshev collocation method, that is, the equations are forced to be satisfied at all Chebyshev radial grid-points, except for the two representing the boundaries. The radial grid points are non-equidistant with

$$
r_{n}=\frac{r_{o}+r_{i}}{2}+x_{n}, \quad n=0, \cdots, N
$$

at which the Chebyshev grid-points $x_{n}$ are defined as

$$
x_{n}=\cos \left(\frac{n \pi}{N}\right)
$$

As a rule of thumb, the spectral maximum to cut-off ratio of the kinetic and magnetic energy is required to be $10^{2}$ for well-resolved dynamo models (Christensen et al. 1999). However, this requirement is not fulfilled for the most chaotic dynamo investigated here. In this case, I follow Kutzner and Christensen (2002) who have shown that the principal structure of the flow and the magnetic field at larger length scales does not sensitively depend on the truncation of the spectrum at the harmonic degree $l_{\max }=85$. 



\section{Mean-field theory}

In the following brief introduction to the mean-field concept, emphasis is placed on the discussion of assumptions and approximations, which allow a rather simple and very elegant treatment of the dynamo problem. Therefore, it is no surprise that they are frequently applied, and it is one aim of this work to test their validity and applicability. In the following, I refer to a spherical coordinate system $(r, \vartheta, \varphi)$ with its polar axis coinciding with the rotation axis of the shell if not otherwise identified. Difficulties due to the use of spherical coordinates and implications resulting from the underlying symmetry properties are pointed out in Section 3.2 and Section 3.4, respectively.

\subsection{The mean-field concept}

Mean-field theory is a statistical theory. Relevant vector fields, such as the velocity or the magnetic field, are therefore subject to an averaging. Though mean fields are a matter of definition, the choice of an averaging is not totally arbitrary. In order to follow the well established line of mean-field dynamo theory (Krause and Rädler 1980, Rädler 1980, Moffatt 1978), the averaging procedure must satisfy the Reynolds rules:

$$
\overline{F+H}=\bar{F}+\bar{H}, \quad \overline{F \bar{H}}=\bar{F} \bar{H}
$$

with arbitrary functions $F=F(\boldsymbol{r}, t), H=H(\boldsymbol{r}, t)$, and an overbar refering to mean quantities. Also, the averaging operator should commute with the differentiation and integration in space and time.

Within the scope of this work, the mean-field concept is applied to the induction equation (2.4) only. Subjecting it to a local averaging, which is assumed to fulfil the Reynolds rules (e.g. an ensemble averaging, space or time averaging), yields

$$
\frac{\partial \overline{\boldsymbol{B}}}{\partial t}=\nabla \times(\overline{\boldsymbol{V}} \times \overline{\boldsymbol{B}})+\nabla \times \mathcal{E}+\frac{1}{P m} \nabla^{2} \overline{\boldsymbol{B}}
$$

with the crucial electromotive force

$$
\mathcal{E}=\overline{\boldsymbol{v} \times \boldsymbol{b}}
$$

already mentioned. Here, $\boldsymbol{v}$ and $\boldsymbol{b}$ are defined by

$$
\boldsymbol{B}=\overline{\boldsymbol{B}}+\boldsymbol{b} \quad \boldsymbol{V}=\overline{\boldsymbol{V}}+\boldsymbol{v}
$$


If $\boldsymbol{V}$ is given, the calculation of $\mathcal{E}$ requires the knowledge of the residual magnetic field $\boldsymbol{b}$, which is governed by

$$
\frac{\partial \boldsymbol{b}}{\partial t}=\nabla \times(\overline{\boldsymbol{V}} \times \boldsymbol{b})+\nabla \times(\boldsymbol{v} \times \overline{\boldsymbol{B}})+\nabla \times \boldsymbol{G}+\frac{1}{P m} \nabla^{2} \boldsymbol{b}
$$

with $\boldsymbol{G}=\boldsymbol{v} \times \boldsymbol{b}-\overline{\boldsymbol{v} \times \boldsymbol{b}}$. According to (3.3) and (3.5), $\mathcal{E}$ is a functional of $\boldsymbol{v}, \overline{\boldsymbol{V}}$, and $\overline{\boldsymbol{B}}$, which is linear in $\overline{\boldsymbol{B}}$. Consequently, the following representation for $\mathcal{E}$ with some integral kernel $\boldsymbol{K}$ has to apply

$$
\mathcal{E}_{i}=\mathcal{E}_{i}^{(0)}+\iint K_{i j}\left(\boldsymbol{x}, \boldsymbol{x}^{\prime}, t, t^{\prime}\right) \bar{B}_{j}\left(\boldsymbol{x}^{\prime}, t^{\prime}\right) d^{3} x^{\prime} d t^{\prime}
$$

in which I refer to Cartesian coordinates for the moment. With the additional assumption that $\boldsymbol{b}$ vanishes if $\overline{\boldsymbol{B}}$ does so, which of course has to be tested, one may conclude that $\mathcal{E}$ is also homogeneous in $\overline{\boldsymbol{B}}$, i.e. $\mathcal{E}^{(0)} \equiv 0$. In principle, one could continue now determining $\boldsymbol{K}$ and using relation (3.6) to integrate the dynamo equation (3.2). However, this is usually not done, and mean-field dynamo theory would loose lots of its simplicity and attractiveness proceeding this way. Instead, $\mathcal{E}$ is assumed to depend instantaneously and nearly locally on $\overline{\boldsymbol{B}}$. Therefore, $\overline{\boldsymbol{B}}$ in (3.6) may be replaced by its Taylor series expansion at $\boldsymbol{x}$

$$
\bar{B}_{j}\left(\boldsymbol{x}^{\prime}, t\right)=\bar{B}_{j}(\boldsymbol{x}, t)+\left(x_{k}^{\prime}-x_{k}\right) \frac{\partial \bar{B}_{j}(\boldsymbol{x}, t)}{\partial x_{k}}+\cdots
$$

and taken out of the integral:

$$
\mathcal{E}_{i}=a_{i j} \bar{B}_{j}+b_{i j k} \frac{\partial \bar{B}_{j}}{\partial x_{k}}+\cdots
$$

with

$$
\begin{aligned}
a_{i j} & =\iint K_{i j}\left(\boldsymbol{x}, \boldsymbol{x}^{\prime}, t, t^{\prime}\right) d^{3} x^{\prime} d t^{\prime} \\
b_{i j k} & =\iint K_{i j}\left(\boldsymbol{x}, \boldsymbol{x}^{\prime}, t, t^{\prime}\right)\left(x_{k}^{\prime}-x_{k}\right) d^{3} x^{\prime} d t^{\prime}
\end{aligned}
$$

It is this Taylor series expansion in which the assumption of a scale separation comes into play: $\boldsymbol{K}$ is assumed to be markedly different from zero for small $\left|\boldsymbol{x}^{\prime}-\boldsymbol{x}\right|$ and $\left|t^{\prime}-t\right|$ only. Hence, the expansion in (3.8) is expected to converge rapidly, and it is argued that only spatial derivatives up to the first order need to be taken into account - sometimes the expansion in (3.8) is even truncated after the first term. This is an important simplification, which accounts a lot for the applicability of mean-field theory. However, it is not obvious whether the expansion in (3.8) converges at all.

Slightly different from the traditional approach described above, a non-local average is adopted in this work. In order to define a mean vector field, its components with respect to the spherical coordinate system are averaged over all values of the azimuthal coordinate $\varphi$, e.g. $\overline{\boldsymbol{B}}=\bar{B}_{r} \boldsymbol{e}_{r}+\bar{B}_{\vartheta} \boldsymbol{e}_{\vartheta}+\bar{B}_{\varphi} \boldsymbol{e}_{\varphi}$. As a consequence, all mean fields are axisymmetric about the rotation axis from now on. Note that with the definition of mean fields used here, the Reynolds averaging rules apply exactly. 
Due to the non-local $\varphi$-average, some modifications of (3.6-3.10) occur: Instead of (3.6), we have now

$$
\mathcal{E}_{\kappa}=\iint K_{\kappa \lambda}\left(r, \vartheta, r^{\prime}, \vartheta^{\prime}, t, t^{\prime}\right) \bar{B}_{\lambda}\left(r^{\prime}, \vartheta^{\prime}, t^{\prime}\right) r^{\prime} \sin \vartheta^{\prime} d r^{\prime} d \vartheta^{\prime} d t^{\prime}
$$

at which $\mathcal{E}^{(0)}$ has been already ignored. Inserting the Taylor series expansion

$$
\bar{B}_{\lambda}\left(r^{\prime}, \vartheta^{\prime}, t^{\prime}\right)=\bar{B}_{\lambda}(r, \vartheta, t)+\left(r^{\prime}-r\right) \frac{\partial \bar{B}_{\lambda}(r, \vartheta, t)}{\partial r}+\left(\vartheta^{\prime}-\vartheta\right) \frac{\partial \bar{B}_{\lambda}(r, \vartheta, t)}{r \partial \vartheta}+\cdots
$$

we finally arrive at

$$
\mathcal{E}_{\kappa}=\tilde{a}_{\kappa \lambda} \bar{B}_{\lambda}+\tilde{b}_{\kappa \lambda r} \frac{\partial \bar{B}_{\lambda}}{\partial r}+\tilde{b}_{\kappa \lambda \vartheta} \frac{1}{r} \frac{\partial \bar{B}_{\lambda}}{\partial \vartheta}+\cdots
$$

with

$$
\begin{aligned}
\tilde{a}_{\kappa \lambda} & =\int K_{\kappa \lambda}\left(r, \vartheta, r^{\prime}, \vartheta^{\prime}, t, t^{\prime}\right) r^{\prime} \sin \vartheta^{\prime} d r^{\prime} d t^{\prime} \\
\tilde{b}_{\kappa \lambda r} & =\int K_{\kappa \lambda}\left(r, \vartheta, r^{\prime}, \vartheta^{\prime}, t, t^{\prime}\right)\left(r^{\prime}-r\right) r^{\prime} \sin \vartheta^{\prime} d r^{\prime} d \vartheta^{\prime} d t^{\prime} \\
\tilde{b}_{\kappa \lambda \vartheta} & =\int K_{\kappa \lambda}\left(r, \vartheta, r^{\prime}, \vartheta^{\prime}, t, t^{\prime}\right)\left(\vartheta^{\prime}-\vartheta\right) r^{\prime} \sin \vartheta^{\prime} d r^{\prime} d \vartheta^{\prime} d t^{\prime}
\end{aligned}
$$

Let us assume that only up to first order derivatives are considered in (3.13). Inserting relation (3.13) into (3.2) then keeps the character of the dynamo equation unchanged. It remains a linear parabolic partial differential equation, but now with 27 unknown parameters $\tilde{a}_{\kappa \lambda}, \tilde{b}_{\kappa \lambda r}$ and $\tilde{b}_{\kappa \lambda \vartheta}$. These so called mean-field coefficients are thought to be decisive for dynamo action, and it is an outstanding task and one aim of this project to determine them properly.

An important tool to derive simple results for a reduced number of mean-field coefficients has been the second order correlation approximation which results in neglecting the term $\nabla \times G$ in (3.5). But before I outline these well known results, it is necessary to discuss specific problems which come along with the use of non-Cartesian coordinates adjusted to the spherical geometry.

\subsection{Mean-field coefficients in spherical geometry}

The expansion of $\mathcal{E}$ referring to partial derivatives as given in (3.13) is not covariant, that is, the mean-field coefficients $\tilde{a}_{\kappa \lambda}, \tilde{b}_{\kappa \lambda r}, \tilde{b}_{\kappa \lambda \vartheta}$ should not be interpreted as tensor components. However, it is of course indispensable to establish a coordinate-independent connection between the vectors $\mathcal{E}$ and $\bar{B}$ in order to relate the mean-field coefficients to physical dynamo-effects. Following this aim, (3.13) has to be rewritten with the partial derivatives replaced by the covariant derivative:

$$
\mathcal{E}^{\kappa}=\hat{a}_{\lambda}^{\kappa} \bar{B}^{\lambda}+\hat{b}_{\lambda}^{\kappa}{ }_{\lambda}^{\mu} \bar{B}_{; \mu}^{\lambda}
$$


in which

$$
\bar{B}_{; \mu}^{\lambda}=\frac{\partial \bar{B}^{\lambda}}{\partial x^{\mu}}+\Gamma_{\mu \nu}^{\lambda} \bar{B}^{\nu}
$$

and the Christoffel symbols $\Gamma_{\mu \nu}^{\lambda}$ are defined as

$$
\Gamma_{\mu \nu}^{\lambda}=\frac{1}{2} g^{\lambda \xi}\left(\frac{\partial g_{\xi \mu}}{\partial x^{\nu}}+\frac{\partial g_{\xi \nu}}{\partial x^{\mu}}+\frac{\partial g_{\nu \mu}}{\partial x^{\xi}}\right)
$$

Here, $g_{\mu \nu}$ denotes the metric tensor, and $x^{\mu}, \mu=1, \cdots, 3$, stands for $r, \vartheta$, and $\varphi$, respectively; some caution has to be taken since (3.17-3.18) refer to a non-normalised basis. Any coordinate transformation applied to (3.17) yields

$$
\begin{aligned}
\mathcal{E}^{\prime i} & =\frac{\partial x^{\prime i}}{\partial x^{\kappa}}\left(\hat{a}_{\lambda}^{\kappa} \bar{B}^{\lambda}+\hat{b}_{\lambda}^{\kappa}{ }_{\lambda}^{\mu} \bar{B}_{; \mu}^{\lambda}\right) \\
& =\frac{\partial x^{\prime i}}{\partial x^{\kappa}} \hat{a}_{\lambda}^{\kappa} \frac{\partial x^{\lambda}}{\partial x^{\prime j}} \frac{\partial x^{\prime j}}{\partial x^{\mu}} \bar{B}^{\mu} \\
& +\frac{\partial x^{i}}{\partial x^{\xi}} \frac{\partial x^{\rho}}{\partial x^{\prime j}} \frac{\partial x^{\prime k}}{\partial x^{\sigma}} \hat{b}_{\rho}^{\xi \sigma} \frac{\partial x^{\tau}}{\partial x^{\prime k}} \frac{\partial x^{\prime l}}{\partial x^{\rho}} \bar{B}_{; \rho}^{\tau} \\
& =\hat{a}_{j}^{i}{ }^{\prime \prime}{ }^{\prime j}+\hat{b}_{j}^{\prime i}{ }_{j}^{k} \bar{B}_{; k}^{\prime j}
\end{aligned}
$$

which proves the actual covariance of (3.17). For spherical coordinates, axisymmetric $\overline{\boldsymbol{B}}$, and for a normalised basis, relation (3.17) reads

$$
\begin{aligned}
\mathcal{E}_{\kappa}= & a_{\kappa r} \bar{B}_{r}+a_{\kappa \vartheta} \bar{B}_{\vartheta}+a_{\kappa \varphi} \bar{B}_{\varphi} \\
& +b_{\kappa r r} \partial_{r} \bar{B}_{r}+b_{\kappa r \vartheta}(1 / r)\left(\partial_{\vartheta} \bar{B}_{r}-\bar{B}_{\vartheta}\right)-b_{\kappa r \varphi}(1 / r) \bar{B}_{\varphi} \\
& +b_{\kappa \vartheta r} \partial_{r} \bar{B}_{\vartheta}+b_{\kappa \vartheta \vartheta}(1 / r)\left(\partial_{\vartheta} \bar{B}_{\vartheta}+\bar{B}_{r}\right)-b_{\kappa \vartheta \varphi}(\cot \vartheta / r) \bar{B}_{\varphi} \\
& +b_{\kappa \varphi r} \partial_{r} \bar{B}_{\varphi}+b_{\kappa \varphi \vartheta}(1 / r) \partial_{\vartheta} \bar{B}_{\varphi}+b_{\kappa \varphi \varphi}(1 / r)\left(\bar{B}_{r}+\cot \vartheta \bar{B}_{\vartheta}\right)
\end{aligned}
$$

On the other hand, (3.17) may be likewise written coordinate-independent as ${ }^{1}$

$$
\mathcal{E}=\boldsymbol{a} \overline{\boldsymbol{B}}+\boldsymbol{b} \nabla \overline{\boldsymbol{B}}
$$

Such as every tensor, $\boldsymbol{a}$ can be separated in its symmetric and antisymmetric parts

$$
\boldsymbol{a}=\boldsymbol{a}^{(\mathrm{sy})}+\boldsymbol{a}^{(\mathrm{as})}
$$

at which the symmetric part is traditionally linked to the $\alpha$-tensor,

$$
\alpha_{\kappa \lambda}=-a_{\kappa \lambda}^{(\mathrm{sy})}=-\frac{1}{2}\left(a_{\kappa \lambda}+a_{\lambda \kappa}\right)
$$

while the antisymmetric part is adequately represented by a vector $\gamma$

$$
a_{\kappa \lambda}^{(\mathrm{as})}=\epsilon_{\kappa \lambda \mu} \gamma_{\mu}
$$

\footnotetext{
${ }^{1}$ The tensors $\boldsymbol{a}$ and $\boldsymbol{b}$ are now defined for a non-local $\varphi$-average and have to be distinguished from those given in (3.9-3.10). However, the notation shall suggest that they all are coefficients in a covariant expansion of $\mathcal{E}$.
} 
By the same token, the second-rank gradient tensor $\nabla \overline{\boldsymbol{B}}$ may be split in its symmetric and antisymmetric parts

$$
\boldsymbol{b} \nabla \overline{\boldsymbol{B}}=-\boldsymbol{c}(\nabla \times \overline{\boldsymbol{B}})-\boldsymbol{\kappa}(\nabla \overline{\boldsymbol{B}})^{(\mathrm{sy})}
$$

and hereafter equally the second-rank tensor $c$, so that we finally arrive at

$$
\boldsymbol{b} \nabla \overline{\boldsymbol{B}}=-\boldsymbol{\beta}(\nabla \times \overline{\boldsymbol{B}})-\boldsymbol{\delta} \times(\nabla \times \overline{\boldsymbol{B}})-\boldsymbol{\kappa}(\nabla \overline{\boldsymbol{B}})^{(\mathrm{sy})}
$$

Altogether, we obtain the well established expression for the mean-electromotive force

$$
\mathcal{E}=-\boldsymbol{\alpha} \overline{\boldsymbol{B}}-\boldsymbol{\gamma} \times \overline{\boldsymbol{B}}-\boldsymbol{\beta}(\nabla \times \overline{\boldsymbol{B}})-\boldsymbol{\delta} \times(\nabla \times \overline{\boldsymbol{B}})-\boldsymbol{\kappa}(\nabla \overline{\boldsymbol{B}})^{(\mathrm{sy})}
$$

in which $\boldsymbol{\alpha}$ and $\boldsymbol{\beta}$ are symmetric tensors of second rank, and $\boldsymbol{\kappa}$ is a third-rank tensor (Rädler 1995). While $\alpha$ describes the classical $\alpha$-effect, the $\gamma$-term contributes to the transport of mean magnetic flux in addition to that by a mean motion. Therefore, the action of the $\gamma$-term is often called turbulent or magnetic pumping (e.g. Ossendrijver et al. 2002). The symmetric $\beta$-tensor gives rise to an anisotropic turbulent diffusion and dominates the molecular one in most cases. Finally, there is a further inductive dynamo effect related to the $\delta$-term as first noticed by Rädler (1969a,b), the $\delta \times j$-effect, which is also known as shear-current effect (e.g. Gubbins 1974, Stix 1976, Rogachevskii and Kleeorin 2003, 2004). It results in an electromotive force parallel to $\boldsymbol{\delta} \times(\nabla \times \overline{\boldsymbol{B}})$ caused by an anisotropic turbulent conductivity with a conductivity tensor which is no longer symmetric and resembles the Hall effect in some respect. The $\delta \times j$-effect is of particular interest in the context of the closure of the dynamo cycle: Together with a differential rotation, a closed dynamo cycle may be envisaged as has been demonstrated by Rädler (1969b). But, its astrophysical significance has not been proven yet. All additional terms in the expansion of $\mathcal{E}$, e.g. the $\kappa$-terms, are more difficult to interpret.

The tensors $\boldsymbol{a}$ and $\boldsymbol{b}$ occurring in (3.21) are related to the tensors $\boldsymbol{\alpha}, \boldsymbol{\gamma}, \boldsymbol{\beta}, \boldsymbol{\delta}$ and $\boldsymbol{\kappa}$ in (3.28) by

$$
\begin{aligned}
\alpha_{\kappa \lambda} & =-1 / 2\left(a_{\kappa \lambda}+a_{\lambda \kappa}\right) \\
\gamma_{\kappa} & =1 / 2 \epsilon_{\kappa \lambda \mu} a_{\lambda \mu} \\
\beta_{\kappa \lambda} & =1 / 4\left(\epsilon_{\kappa \mu \nu} b_{\lambda \mu \nu}+\epsilon_{\lambda \mu \nu} b_{\kappa \mu \nu}\right) \\
\delta_{\kappa} & =-1 / 4\left(b_{\lambda \kappa \lambda}-b_{\lambda \lambda \kappa}\right) \\
\kappa_{\kappa \lambda \mu} & =-1 / 2\left(b_{\kappa \lambda \mu}+b_{\kappa \mu \lambda}\right) .
\end{aligned}
$$

But how are the covariant tensor components in (3.21) or (3.28) linked to the mean-field coefficients appearing in the non-covariant expansion of the mean electromotive force in (3.13)? From a comparison of (3.21) with an expansion of $\mathcal{E}$ due to partial derivatives

$$
\begin{aligned}
\mathcal{E}_{\kappa}= & \tilde{a}_{\kappa r} \bar{B}_{r}+\alpha_{\kappa \vartheta} \bar{B}_{\vartheta}+\tilde{a}_{\kappa \varphi} \bar{B}_{\varphi} \\
& +\tilde{b}_{\kappa r r} \partial_{r} \bar{B}_{r}+\tilde{b}_{\kappa r \vartheta}(1 / r) \partial_{\vartheta} \bar{B}_{r} \\
& +\tilde{b}_{\kappa \vartheta r} \partial_{r} \bar{B}_{\vartheta}+\tilde{b}_{\kappa \vartheta \vartheta}(1 / r) \partial_{\vartheta} \bar{B}_{\vartheta} \\
& +\tilde{b}_{\kappa \varphi r} \partial_{r} \bar{B}_{\varphi}+\tilde{b}_{\kappa \varphi \vartheta}(1 / r) \partial_{\vartheta} \bar{B}_{\varphi}
\end{aligned}
$$


it is straight forward to conclude

$$
\begin{aligned}
a_{\kappa r}+b_{\kappa \varphi \varphi} / r & =\tilde{a}_{\kappa r}-\tilde{b}_{\kappa \vartheta \vartheta} / r \\
a_{\kappa \vartheta}+\cot \vartheta b_{\kappa \varphi \varphi} / r & =\tilde{a}_{\kappa \vartheta}+\tilde{b}_{\kappa r \vartheta} / r \\
a_{\kappa \varphi}-\left(b_{\kappa r \varphi}+\cot \vartheta b_{\kappa \vartheta \varphi}\right) / r & =\tilde{a}_{\kappa \varphi} \\
b_{\kappa \lambda r} & =\tilde{b}_{\kappa \lambda r} \\
b_{\kappa \lambda \vartheta} & =\tilde{b}_{\kappa \lambda \vartheta}
\end{aligned}
$$

Here, we encounter a principal problem. Due to (3.35-3.39), the components of the tensors $\boldsymbol{a}$ and $\boldsymbol{b}$ are not well-defined. In contrast, only the terms $a_{\kappa r}+b_{\kappa \varphi \varphi}, a_{\kappa \vartheta}+\cot \vartheta b_{\kappa \varphi \varphi} / r$ and $a_{\kappa \varphi}-\left(b_{\kappa r \varphi}+\cot \vartheta b_{\kappa \vartheta \varphi}\right) / r$ are unique and thus independent quantities. This ambiguity is a consequence of the azimuthal symmetry, which reduces the number of mean-field coefficients in (3.34) since all partial derivatives with respect to the coordinate $\varphi$ vanish and the coefficients $\tilde{b}_{\kappa \lambda \varphi}$ remain undetermined. However, setting $\tilde{b}_{\kappa \lambda \varphi}=0$ equally compels $b_{\kappa \lambda \varphi}=0$. This seems to be the most natural choice and leads to a unique solution of (3.353.39). On account of (3.29-3.33), we may derive relations between $\alpha_{\kappa \lambda}, \gamma_{\kappa}, \cdots, \kappa_{\kappa \lambda \mu}$ and $\tilde{a}_{\kappa, \lambda}, \tilde{b}_{\kappa \lambda \mu}$ which are listed in Appendix A.

\subsection{The second order correlation approximation}

Most of the well-known results about mean-field coefficients rely on the second order correlation approximation (SOCA) which is likewise called first order smoothing approximation (FOSA). Let us recall that equation (3.5) has to be solved in order to determine $\mathcal{E}$. This requires the application of a closure method, and the simplest one is to neglect the term $\nabla \times G$ in (3.5), which implies to consider only up to second-order correlations of fluctuating quantities.

An order-of-magnitude estimation reveals two circumstances when this neglect, i.e. SOCA, would appear to be justified (Krause and Rädler 1980, Moffatt 1978, Stix 2002). The first one can be summarised by the condition $R m \ll 1$, which ensures that $\nabla \times$ $G$ is small compared to the diffusion term. The second one is characterised by $S t=$ $u \tau / l \ll 1$ at which $\mathrm{u}$ is a typical magnitude of $\boldsymbol{V}$, and $l$ and $\tau$ are typical scales of the variation of $\boldsymbol{V}$ and $\boldsymbol{b}$ in space and time. The dimensonless number $S t$ is sometimes called Strouhal number (Krause and Rädler 1980). If $S t \ll 1$ holds, then $\nabla \times \boldsymbol{G}$ is negligible in comparison to $\partial \boldsymbol{b} / \partial t$.

Unfortunately, neither of the two conditions seems to be satisfied in most astrophysical applications. Therefore, it will be important to learn in which respect the actual meanfield coefficients deviate from those derived in the SOCA-approximation and in which way this difference affects corresponding dynamo calculations.

Besides the SOCA-approximation, further simplifications are necessary to end up with simple expressions for $\boldsymbol{\alpha}$ and $\boldsymbol{\beta}$. The mean flow in (3.6), for instance, is usually also neglected, and, maybe even more dramatic, $\boldsymbol{v}$ is frequently assumed to be statistically homogeneous and isotropic ${ }^{2}$ in order to reduce the number of non-vanishing tensor components. Then, the most commonly adopted result for the $\alpha$-tensor in the high-conductivity

\footnotetext{
${ }^{2} \mathrm{~A}$ vector field is statistically homogeneous and isotropic if all averages derived from it are invariant under translation and rotation.
} 
limit reads $\boldsymbol{\alpha}=\alpha_{I} \delta_{\lambda \kappa}$, with

$$
\alpha_{I}=-\frac{1}{3} \overline{\boldsymbol{v} \cdot(\nabla \times \boldsymbol{v})} \tau_{c}
$$

and $\tau_{c}$ being the correlation time defined by

$$
\int_{0}^{\infty} \overline{\boldsymbol{v}(\boldsymbol{r}, t) \cdot(\nabla \times \boldsymbol{v}(\boldsymbol{r}, t-\tau))} d \tau=\overline{\boldsymbol{v}(\boldsymbol{r}, t) \cdot(\nabla \times \boldsymbol{v}(\boldsymbol{r}, t))} \tau_{c}
$$

In the same way, it follows $\boldsymbol{\beta}=\beta_{I} \delta_{\lambda \kappa}$, with

$$
\beta_{I}=\frac{1}{3} \overline{\boldsymbol{v}^{2}} \tau_{c}
$$

Note, that in addition to all simplifications already mentioned above, (3.40) and (3.42) apply at best, if $q:=\lambda_{c}^{2} / \tau_{c} \eta \rightarrow \infty$, in which $\lambda_{c}$ denotes a typical length scale of the velocity field. Here, the dimensionless parameter $q$ can be interpreted as the ratio of the timescales related to variations in the magnetic field and velocity field, respectively. If on the other hand $q$ becomes very small in the low conductivity limit, relations (3.40) and (3.42) have to be replaced by

$$
\alpha_{I}=-\frac{1}{3} P m \overline{\boldsymbol{a} \cdot(\nabla \times \boldsymbol{a})}
$$

and

$$
\beta_{I}=\frac{1}{3} P m \overline{\boldsymbol{a}^{2}}
$$

in which the vector potential $\boldsymbol{a}$ is defined by $\boldsymbol{v}=\nabla \times \boldsymbol{a}$ and $\nabla \cdot \boldsymbol{a}=0$, supposing that $\boldsymbol{v}$ is solenoidal (Krause and Rädler 1980). Although relations (3.40-3.44) are very appealing because of their simplicity, they are crude simplifications and their validity is severely limited as will be shown in this work.

\subsection{Symmetry properties of mean-field coefficients}

The symmetry properties of a self-gravitating rotating sphere as well as those of the full set of MHD equations have a major effect on the mean-field coefficients and therefore on the possible dynamo solutions. The spherical symmetry of a gravitating sphere, or likewise a spherical shell, is broken due to the spinning about its polar axis, which has only axial symmetry. Following Gubbins and Zhang (1993), the remaining symmetry transformations, i.e. transformations which take the rotating sphere into itself, are a reflection in the equatorial plane, $E$, a rotation about the polar axis by an angle $2 \pi / M$, $P_{M}$, where $M$ is an integer, and finally a combination of both, $O_{M}=E \circ P_{M}$, and the identical transformation, I. Altogether, these symmetry transformations form a Lie group, $L_{S}=\left\{I, E, O_{M}, P_{M} / M \in \mathbb{N}\right\}$. The symmetry group $L_{S}$ constrains the symmetry properties of a convection driven flow. In particular, except for $P_{M}, L_{S}$ does not include any further rotations nor any translations. Therefore, one has to assume that the velocity-dependent $\alpha$-tensor in its most general representation, considered inside a rotating spherical shell at radius $r$, will depend on at least two axis, $\hat{\boldsymbol{r}}$ and $\hat{\boldsymbol{p}}$. Here, $\hat{\boldsymbol{r}}$ denotes 
a unit vector in radial direction while $\hat{\boldsymbol{p}}$ is parallel to the rotation axis. A corresponding representation for a tensor of second rank is derived in Appendix B and can be written as $a_{i j}=S_{i j}+A_{i j}$ with its symmetric and antisymmetric parts

$$
\begin{aligned}
S_{i j}= & a_{1} \delta_{i j}+a_{2} \hat{r}_{i} \hat{r}_{j}+a_{3} \hat{p}_{i} \hat{p}_{j}+a_{4}\left(\hat{r}_{i} \hat{p}_{j}+\hat{r}_{j} \hat{p}_{i}\right) \\
& +a_{5}\left[\hat{r}_{i}(\hat{\boldsymbol{p}} \times \hat{\boldsymbol{r}})_{j}+\hat{r}_{j}(\hat{\boldsymbol{p}} \times \hat{\boldsymbol{r}})_{i}\right]+a_{6}\left[\hat{p}_{i}(\hat{\boldsymbol{p}} \times \hat{\boldsymbol{r}})_{j}+\hat{p}_{j}(\hat{\boldsymbol{p}} \times \hat{\boldsymbol{r}})_{i}\right] \\
A_{i j}= & a_{7} \epsilon_{i j k} \hat{r}_{k}+a_{8} \epsilon_{i j k} \hat{p}_{k}+a_{9} \epsilon_{i j k}(\hat{\boldsymbol{p}} \times \hat{\boldsymbol{r}})_{k}
\end{aligned}
$$

Choosing a spherical basis and the spherical coordinates $(r, \vartheta, \varphi)$, we have $\hat{\boldsymbol{r}}=(1,0,0)$ and $\hat{\boldsymbol{p}}=(\cos \vartheta,-\sin \vartheta, 0)$, which leads to the matrix representations

$$
\boldsymbol{S}=\left(\begin{array}{ccc}
a_{1}+a_{2}+a_{3} \cos ^{2} \vartheta & -a_{3} \cos \vartheta \sin \vartheta & a_{5} \sin \vartheta \\
+2 a_{4} \cos \vartheta & -a_{4} \sin \vartheta & +a_{6} \cos \vartheta \sin \vartheta \\
-a_{3} \cos \vartheta \sin \vartheta-a_{4} \sin \vartheta & a_{1}+a_{3} \sin ^{2} \vartheta & -a_{6} \sin ^{2} \vartheta \\
a_{5} \sin \vartheta+a_{6} \cos \vartheta \sin \vartheta & -a_{6} \sin ^{2} \vartheta & a_{1}
\end{array}\right)
$$

and

$$
\boldsymbol{A}=\left(\begin{array}{ccc}
0 & -a_{9} \sin \vartheta & a_{8} \sin \vartheta \\
-a_{9} \sin \vartheta & 0 & a_{7}+a_{8} \cos \vartheta \\
-a_{8} \sin \vartheta & -a_{7}-a_{8} \cos \vartheta & 0
\end{array}\right)
$$

The $a_{i}, i=1, \cdots 9$, may be any functions of $r$ and $\cos \vartheta$. Somewhat disappointing, none of the tensor components vanishes beforehand because of the underlying symmetry properties. However, it is instructive to note that the azimuthal invariance causes $\alpha_{\varphi \varphi}$ to represent the isotropic part of the tensor.

The importance of symmetries for the dynamo problem can be immediately realised if in contrast to (3.45) and (3.46) the representation of the $\alpha$-tensor is allowed to depend only on one preferred direction $\hat{z}$. This condition is fulfilled for the Roberts flow (Roberts 1972), which is often applied to investigate $\alpha^{2}$-dynamos (Rädler et al. 2002, Tilgner 1997, 2004). The Roberts flow is a spatially periodic, helical flow in an infinite domain and stays invariant under affine transformations in the $(x, y)$-plane (Rädler et al. 2002, Feudel et al. 2003). This ensures that the tensor $a_{i j}$ is a linear combination of $\delta_{i j}, \hat{z}_{i} \hat{z}_{j}$ and $\epsilon_{i j k} \hat{z}_{k}$ only,

$$
a_{i j}=a_{1} \delta_{i j}+a_{2} \hat{z}_{i} \hat{z}_{j}+\gamma \epsilon_{i j k} \hat{z}_{k}
$$

Here the coefficients $a_{1}, a_{2}, \gamma$ are simply constants and a comparison between (3.49) and (3.45-3.46) reveals to which extent the representation of a second-rank tensor has already simplified. Therefore, results derived for the Roberts flow cannot be generalised without some caution.

Further constraints are given by the symmetry properties of the MHD equations. It is well known that the induction equation allows for solutions which separate in an equatorial-symmetric, $E^{S}$, and an equatorial-antisymmetric family, $E^{A}$. While $E^{S}$ vectors transform under reflection at the equatorial plane as

$$
\left[S_{r}, S_{\vartheta}, S_{\varphi}\right](r, \vartheta, \varphi)=\left[S_{r},-S_{\vartheta}, S_{\varphi}\right](r, \pi-\vartheta, \varphi)
$$


for an $E^{A}$ vector the transformation rule

$$
\left[S_{r}, S_{\vartheta}, S_{\varphi}\right](r, \vartheta, \varphi)=\left[-S_{r}, S_{\vartheta},-S_{\varphi}\right](r, \pi-\vartheta, \varphi)
$$

applies. Any vector $\boldsymbol{B}$ may be decomposed into both parts, $E^{S}$ and $E^{A}$. This can easily be seen: Let $\boldsymbol{B}^{\prime}$ denote the result of reflecting $\boldsymbol{B}$ in the equatorial plane. The quantities $\boldsymbol{B}^{\boldsymbol{S}}=1 / 2\left(\boldsymbol{B}+\boldsymbol{B}^{\prime}\right)$ and $\boldsymbol{B}^{\boldsymbol{A}}=1 / 2\left(\boldsymbol{B}-\boldsymbol{B}^{\prime}\right)$ are of $E^{S}$ and $E^{A}$ type, respectively, and $\boldsymbol{B}=\boldsymbol{B}^{\boldsymbol{S}}+\boldsymbol{B}^{\boldsymbol{A}}$. The linear terms in the induction equation, $\partial \boldsymbol{B} / \partial t$ and $\eta \nabla^{2} \boldsymbol{B}$, transform in the same way as $\boldsymbol{B}$. Therefore, it depends on the nonlinear term $\nabla \times(\boldsymbol{V} \times \boldsymbol{B})$ and in particular on the nature of $\boldsymbol{V}$ whether solutions of the induction equation are separable due to their reflectional symmetry-properties. Let $\boldsymbol{V}$ be of $E^{S}$ type. Since the vector operators $\nabla \times$ and $\times$ are both antisymmetric on reflection, their effect on the reflectional symmetry cancels out and the term $\nabla \times(\boldsymbol{V} \times \boldsymbol{B})$ is finally of same symmetry as $\boldsymbol{B}$. On the other hand, a velocity of $E^{A}$ type causes the nonlinear term $\nabla \times(\boldsymbol{V} \times \boldsymbol{B})$ to be always of opposite symmetry as $\boldsymbol{B}$. As a consequence, separable and even independent solutions of the induction equation with respect to the symmetry families $E^{S}$ and $E^{A}$ exist as long as the velocity field is equatorial symmetric. Thus, given $\boldsymbol{V}$ of $E^{S}$ type and starting with $\boldsymbol{B}$ of $E^{A}$ symmetry, a solution of the induction equation will always be of pure $E^{A}$ type and vice versa for an initial field of $E^{S}$ symmetry.

Note that it is important to distinguish between independent and separable solutions. Independent solutions are typical features of linear equations and can exist without influencing each other. Though the full set of MHD equations allows separable solutions, they are in general not independent (Gubbins and Zhang 1993). Mixing both types of solutions leads to $E^{A}$ terms in the momentum equation which are induced by the nonlinear Lorentz force $\left(J^{S}+J^{A}\right) \times\left(B^{S}+B^{A}\right)$. At the same time, these terms must be balanced by $E^{A}$ velocities and $E^{A}$ temperatures, which finally also couple $E^{S}$ and $E^{A}$ solutions of $\boldsymbol{B}$ in the induction equation.

Besides the reflectional symmetry, the MHD equations are invariant under field reversal $i: \boldsymbol{B} \rightarrow-\boldsymbol{B}$. In order to prove this it is sufficient to note that the Lorentz force in the momentum equation is quadratic in $\boldsymbol{B}$ and the induction equation is homogeneous in $\boldsymbol{B}$. Both solutions exist in the case of the geodynamo, and it is still an outstanding task to understand the physical mechanism which triggers a polarity transition. Since some paleomagnetic data suggest that the polarity-transition phase is dominated by the equatorialsymmetric family (Merrill et al. 1998) while the endpoints of the transition belong to the family of $E^{A}$ solutions, reversals can possibly be understood as symmetry-breaks of an $E^{A}$ solution by an $E^{S}$ one.

A further symmetry should be mentioned: Both, the set of MHD-equations and the rotating sphere are unchanged under an arbitrary rotation about the polar axis. Although an axisymmetric dynamo solution is thus consistent with the given symmetry constraints, the existence of a steady axisymmetric solution is ruled out by the famous Cowling theorem.

How are the reflectional symmetry-properties of the velocity field and the induction equation linked to the symmetries of the mean-field coefficients? Similarly to the induction equation, solutions of the mean-field dynamo equation separate in equatorialsymmetric and antisymmetric fields if the velocity is of $E^{S}$ type. In this case, the additional term, $\nabla \times \mathcal{E}=\nabla \times \overline{\boldsymbol{v} \times \boldsymbol{b}}$, is always of same symmetry as $\overline{\boldsymbol{B}}$. As a result, replacing 


\begin{tabular}{|l|l|}
\hline SYMMETRY & MEAN-FIELD COEFFICIENTS \\
\hline \hline \multirow{2}{*}{ equatorial symmetric } & $\begin{array}{l}\tilde{a}_{r \vartheta}, \tilde{a}_{\vartheta r}, \tilde{a}_{\vartheta \varphi}, \tilde{a}_{\varphi \vartheta}, \tilde{b} \\
\tilde{b}_{r r \vartheta}, \tilde{b}_{r \vartheta r}, \tilde{b}_{\vartheta r r}, \tilde{b}_{\varphi r \vartheta}, \tilde{b}_{\varphi \vartheta r}, \tilde{b}_{r \varphi \theta}, \tilde{b}_{\vartheta \vartheta \vartheta}, \tilde{b}_{\vartheta \varphi r}, \tilde{b}_{\varphi \varphi \vartheta}\end{array}$ \\
\hline equatorial antisymmetric & $\begin{array}{l}\tilde{a}_{r r}, \tilde{a}_{r \varphi}, \tilde{a}_{\vartheta \vartheta}, \tilde{a}_{\varphi r}, \tilde{a}_{\varphi \varphi}, \\
\tilde{b}_{r r r}, \tilde{b}_{\vartheta r \vartheta}, \tilde{b}_{\vartheta \vartheta r}, \tilde{b}_{\varphi r r}, \tilde{b}_{r \vartheta \vartheta}, \tilde{b}_{r \varphi r}, \tilde{b}_{\vartheta \varphi \vartheta}, \tilde{b}_{\varphi \vartheta \vartheta}, \tilde{b}_{\varphi \varphi r}\end{array}$ \\
\hline
\end{tabular}

Table 3.1: Mean-field coefficients sorted with respect to their equatorial symmetry in the case of an equatorial-symmetric velocity field

$\mathcal{E}$ by an expansion of the form

$$
\mathcal{E}_{\kappa}=\tilde{a}_{\kappa \lambda} \bar{B}_{\lambda}+\tilde{b}_{\kappa \lambda r} \frac{\partial \bar{B}_{\lambda}}{\partial r}+\tilde{b}_{\kappa \lambda \theta} \frac{1}{r} \frac{\partial \bar{B}_{\lambda}}{\partial \theta}
$$

leads to well defined symmetry predictions for the mean-field coefficients $\tilde{a}_{\kappa \lambda}$ and $\tilde{b}_{\kappa \lambda \mu}$ which are listed in table 3.1. 


\section{How to derive mean-field coefficients}

\subsection{Approach (I): mean-field coefficients based on numer- ically determined electromotive forces}

The principal idea of this approach is to solve the induction equation for the fluctuating field (3.5) in its general form for a given velocity field numerically in order to calculate $\mathcal{E}=\overline{\boldsymbol{v} \times \boldsymbol{b}}$. Subsequently, the linear relation (3.13) between $\mathcal{E}$ and $\overline{\boldsymbol{B}}$ is inverted to determine the mean-field coefficients $\tilde{a}_{\kappa \lambda}, \tilde{b}_{\kappa \lambda \mu}, \cdots$. However, a principal difficulty arises from the number of variables, which exceeds the number of three linear equations provided by the expansion of $\mathcal{E}$. Hence, equation (3.5) is repeatedly solved for various prescribed mean test fields, $\overline{\boldsymbol{B}}_{T}$, but always for the same velocity field, which is held constant. Because mean-field coefficients depend on the considered velocity and magnetic diffusivity only, they must not change according to the choice of $\overline{\boldsymbol{B}}_{\boldsymbol{T}}$. Thus, different electromotive forces corresponding to different test fields $\overline{\boldsymbol{B}}_{T}$ may be combined in order to close the system of linear equations.

In which way this approach is used to determine $\tilde{a}_{i j}$ and $\tilde{b}_{i j k}$ is explained in more detail for stationary velocity fields in Section 4.1.1. Extensions of this method to nonstationary velocity fields and likewise to the derivation of further mean-field coefficients due to higher order derivatives in the expansion of $\mathcal{E}$ are discussed hereafter. For the latter, the choice of the test fields is essential, and Section 4.1.2 is dedicated to this question.

\subsubsection{The method}

First of all, let us consider a stationary velocity field which may be an output of a selfconsistent dynamo calculation. Henceforth, a kinematic viewpoint is taken and the velocity field is held constant.

In order to determine $\mathcal{E}$, the induction equation for the fluctuating field

$$
\frac{\partial \boldsymbol{b}}{\partial t}-\nabla \times(\overline{\boldsymbol{V}} \times \boldsymbol{b})-\nabla \times \boldsymbol{G}-P m^{-1} \nabla^{2} \boldsymbol{b}=\nabla \times\left(\boldsymbol{v} \times \overline{\boldsymbol{B}}_{\boldsymbol{T}}\right)
$$

with

$$
G=v \times b-\overline{v \times b}
$$

has to be solved. Here, $\overline{\boldsymbol{B}}_{T}$ denotes a test field, which appears as a free parameter and has to be chosen. In other words: $\mathcal{E}$ is 'measured' due to the action of a velocity field on 
a prescribed mean test field $\overline{\boldsymbol{B}}_{T}$. Note that

$$
\begin{aligned}
\left(\boldsymbol{V} \times\left(\overline{\boldsymbol{B}}_{\boldsymbol{T}}+\boldsymbol{b}\right)\right)^{\prime} & =\boldsymbol{V} \times\left(\overline{\boldsymbol{B}}_{\boldsymbol{T}}+\boldsymbol{b}\right)-\overline{\boldsymbol{V} \times\left(\overline{\boldsymbol{B}}_{\boldsymbol{T}}+\boldsymbol{b}\right)} \\
& =\boldsymbol{v} \times \overline{\boldsymbol{B}}_{\boldsymbol{T}}+\boldsymbol{G}+\overline{\boldsymbol{V}} \times \boldsymbol{b}
\end{aligned}
$$

Hence, equation (4.1) can be equally written as

$$
\frac{\partial \boldsymbol{b}}{\partial t}=\nabla \times\left(\boldsymbol{V} \times\left(\overline{\boldsymbol{B}}_{\boldsymbol{T}}+\boldsymbol{b}\right)\right)^{\prime}+P m^{-1} \nabla^{2} \boldsymbol{b}
$$

A comparison of (4.3) with the induction equation (2.4) shows that the existing dynamo code (see Chapter 2) can be easily adapted to solve (4.3): Only an additional inhomogeneity $\overline{\boldsymbol{B}}_{T}$ needs to be added during the calculations of the nonlinear products, which are carried out in grid space. At the same time, the axisymmetric parts of $\boldsymbol{B}$ and $\boldsymbol{V} \times\left(\overline{\boldsymbol{B}}_{\boldsymbol{T}}+\boldsymbol{b}\right)$ are set to zero. As soon as the diffusive losses equilibrate the imposed inhomogeneity, (4.3) becomes stationary and the mean electromotive force $\mathcal{E}$ is calculated subsequently in the meridional plane.

Now the expansion of $\mathcal{E}$ including derivatives of $\overline{\boldsymbol{B}}$ up to the first order leads to a system of only three linear equations, but with 27 unknowns $\tilde{a}_{\kappa \lambda}, \tilde{b}_{\kappa \lambda \mu}$. In order to cope with this problem, (4.3) is solved for nine different field configurations $\overline{\boldsymbol{B}}_{\boldsymbol{T}}{ }^{(i)}, i=1, \cdots 9$, and $\mathcal{E}$ is calculated for each of them. The composed system of linear equations can then be expressed symbolically as

$$
\mathcal{E}_{\kappa}^{(i)}=\left(\bar{B}_{T \lambda}^{(i)}, \frac{\partial \bar{B}_{T \lambda}^{(i)}}{\partial x_{\mu}}\right)\left(\begin{array}{c}
\tilde{a}_{\kappa \lambda} \\
\tilde{b}_{\kappa \lambda \mu}
\end{array}\right), \quad i=1, \cdots, 9
$$

Here the coefficient matrix can be advantageously arranged as a diagonal block matrix with three equal blocks for each component of $\mathcal{E}$. Therefore, instead of a $27 \times 27$, rather a $9 \times 9$-system has to be considered, which is solved by LR-decomposition.

How can the above described approach be extended to tackle time-dependent velocity fields? Is there actually the need for a time-dependent solution of (4.3) in order to determine time-dependent mean-field coefficients? On the contrary, one might think of applying the whole procedure explained above iteratively to a time series of snapshots of a given velocity field. For sure, the resulting mean-field coefficients will characterise the dynamo action of the flow at a given time, if it was held constant. But as indicated by the distinction of the high- and low-conductivity limit in Section 3.3, this might not account for the complete time dependency. In fact, the dimensionless parameter $q$ defined in Section 3.3 will always tend to zero in consequence of this approach because the correlation time of the velocity field is assumed to be infinite. Thus, we stick indistinctively to the low conductivity limit, and the resulting mean-field coefficients will be spoiled in this respect.

A general, time-dependent treatment requires to solve equation (4.3) together with (2.1-2.5). Now, the velocity field in (4.3) is updated after every time step leading to a time dependent solution $\boldsymbol{b}=\boldsymbol{b}(t)$. Again, it is assumed that the dependency on the initial value of $\boldsymbol{b}$ is broken up as soon as $\boldsymbol{b}$ and thus $\mathcal{E}$ reach a statistical equilibrium. Having once obtained time-dependent electromotive forces for the various test-fields we proceed as in the stationary case and solve (4.4) for given points in time. 


\begin{tabular}{|c|c|c|c|c|c|c|c|c|c|}
\hline RUN & 1 & 2 & 3 & 4 & 5 & 6 & 7 & 8 & 9 \\
\hline \hline $\bar{B}_{T r}$ & 1 & 0 & 0 & $r$ & 0 & 0 & $\vartheta$ & 0 & 0 \\
$\bar{B}_{T \vartheta}$ & 0 & 1 & 0 & 0 & $r$ & 0 & 0 & $\vartheta$ & 0 \\
$\bar{B}_{T \varphi}$ & 0 & 0 & 1 & 0 & 0 & $r$ & 0 & 0 & $\vartheta$ \\
\hline
\end{tabular}

Table 4.1: Canonic set of test fields used to compute a series of electromotive forces for the determination of $\tilde{a}_{\kappa \lambda}$ and $\tilde{b}_{\kappa \lambda \mu}$.

\subsubsection{The choice of test fields}

There are some constraints on the choice of the test fields. First of all, they have to be linear independent, such that the coefficient matrix in (4.4) does not become singular. Secondly, the test fields must not allow for higher order derivatives unequal zero than considered in the expansion of $\mathcal{E}$, i.e., for the determination of $\tilde{a}_{\kappa \lambda}$ and $\tilde{b}_{\kappa \lambda \mu}$, only constant test fields or test fields linear in $r$ and $\vartheta$ are allowed. A third requirement is of course that all test fields have to be axisymmetric. A canonical set of test fields which fulfils all these demands is given in Table 4.1. Emphasis is placed on the fact that $\overline{\boldsymbol{B}}_{\boldsymbol{T}}$ does not need to be physical. For example, most of the test fields in Table 4.1 are neither divergence free nor fulfil the boundary conditions. But since the mean-field coefficients must not depend on the choice of test fields anyway, they are in particular not affected by the test fields being divergence free or not. However, there is another defect which might cause trouble. The vector fields listed in Table 4.1 do not vanish on the polar axis, and thus $\nabla \times\left(\boldsymbol{v} \times \overline{\boldsymbol{B}}_{\boldsymbol{T}}\right)$ could become singular if the axis was included in the grid and $\boldsymbol{v}$ was different from zero there.

Following the aim to consider up to second order derivatives in the expansion of $\mathcal{E}$, additional 27 mean-field coefficients, $\tilde{c}_{\kappa \lambda r r}, \tilde{c}_{\kappa \lambda r \vartheta}$, and $\tilde{c}_{\kappa \lambda \vartheta \vartheta}$ must be taken into account. This requires 9 more test fields, which may be combined with those listed in Table 4.1:

$$
\begin{aligned}
& \overline{\boldsymbol{B}}_{\boldsymbol{T}}{ }^{(10)}=\left(r^{2}, 0,0\right) \\
& \overline{\boldsymbol{B}}_{\boldsymbol{T}}{ }^{(11)}=\left(0, r^{2}, 0\right) \\
& \overline{\boldsymbol{B}}_{\boldsymbol{T}}{ }^{(12)}=\left(0,0, r^{2}\right) \\
& \overline{\boldsymbol{B}}_{\boldsymbol{T}}{ }^{(13)}=(r \vartheta, 0,0) \\
& \overline{\boldsymbol{B}}_{\boldsymbol{T}}{ }^{(14)}=(0, r \vartheta, 0) \\
& \overline{\boldsymbol{B}}_{\boldsymbol{T}}{ }^{(15)}=(0,0, r \vartheta) \\
& \overline{\boldsymbol{B}}_{\boldsymbol{T}}{ }^{(16)}=\left(\vartheta^{2}, 0,0\right) \\
& \overline{\boldsymbol{B}}_{\boldsymbol{T}}{ }^{(17)}=\left(0, \vartheta^{2}, 0\right) \\
& \overline{\boldsymbol{B}}_{\boldsymbol{T}}{ }^{(18)}=\left(0,0, \vartheta^{2}\right)
\end{aligned}
$$

The constraints on proper test fields mentioned above are quite determinative, and alternatives for those given in Table 4.1 are rare. However, as an experiment, test fields linear in $\vartheta$ have been replaced by fields periodic in $\vartheta$; see Table 4.2 for an example. Even though their partial derivatives with respect to $\vartheta$ do not vanish, they do not exceed unity at least. 


\begin{tabular}{|c|c|c|c|c|c|c|c|c|c|}
\hline RUN & 1 & 2 & 3 & 4 & 5 & 6 & 7 & 8 & 9 \\
\hline \hline $\bar{B}_{T r}$ & 1 & 0 & 0 & $r$ & 0 & 0 & $\cos \vartheta$ & $\sin \vartheta$ & 0 \\
$\bar{B}_{T \vartheta}$ & 0 & 1 & 0 & 0 & $r$ & 0 & $-\sin \vartheta$ & $\cos \vartheta$ & 0 \\
$\bar{B}_{T \varphi}$ & 0 & 0 & $\cos \vartheta$ & 0 & 0 & $r$ & 0 & 0 & $\sin \vartheta$ \\
\hline
\end{tabular}

Table 4.2: Alternative set of test fields used to compute a series of electromotive forces for the determination of $\tilde{a}_{\kappa \lambda}$ and $\tilde{b}_{\kappa \lambda \mu}$.

The resulting mean-field coefficients have been found to equal those obtained by imposing the test fields given in 4.1. This experiment was done in order to confirm that in fact the resulting mean-field coefficients do not depend on the actual choice of the test fields.

In previous attempts, mean fields were imposed via inhomogeneous magnetic boundary conditions, and the induction equation was solved instead of 4.1. A subsequent separation of mean and fluctuating parts enabled then likewise the calculation of $\mathcal{E}$. But for all that, severe difficulties were encountered: The rather uncontrolled evolution of the mean field led to singular coefficient matrices in (4.4) at numerous grid points and to higher order derivatives of $\overline{\boldsymbol{B}}$. These difficulties also occur if a mean field is prescribed as an initial field (Ziegler et al. 1996, Ossendrijver et al. 2002). In contrast, the approach presented here overcomes these problems since it allows for total control over the imposed test fields.

\subsection{Approach (II): analytical determination of mean-field coefficients applying SOCA}

While the first procedure described in the previous section is based on a numerical solution of equation (4.1), a second approach, explained in the following, aims at deriving as far as possible analytical expressions for the components $\tilde{a}_{\kappa \lambda}$. This compels to apply a number of approximations and to rely on specific assumptions, which necessarily constrain the general applicability of this second approach. In particular, SOCA is applied. But still, it serves as an important tool to confirm results obtained with approach (I) and provides some insight into the mathematical structure of mean-field coefficients derived in the low conductivity limit.

Here, we consider again a conducting incompressible fluid in a spherical shell with magnetic diffusivity $\eta$ and electrically insulating boundaries. Further on, we assume that velocity and magnetic field are steady and that there is no mean flow. Then, the induction equation in the second order correlation approximation reads

$$
\eta \nabla^{2} \boldsymbol{b}=-\nabla \times(\boldsymbol{v} \times \overline{\boldsymbol{B}})
$$

Since $\boldsymbol{b}$ is solenoidal, it can be represented as a sum of a poloidal and a toroidal vector in the form

$$
\begin{aligned}
\boldsymbol{b} & =-\nabla \times(\boldsymbol{r} \times \nabla S)-\boldsymbol{r} \times \nabla T \\
& =\left(-\frac{1}{r} \Omega S, \frac{\partial \hat{S}}{\partial \vartheta}+\frac{1}{\sin \vartheta} \frac{\partial T}{\partial \varphi}, \frac{1}{\sin \vartheta} \frac{\partial \hat{S}}{\partial \varphi}-\frac{\partial T}{\partial \vartheta}\right),
\end{aligned}
$$


with

$$
\Omega f=\frac{1}{\sin \vartheta} \frac{\partial}{\partial \vartheta}\left(\sin \vartheta \frac{\partial f}{\partial \vartheta}\right)+\frac{1}{\sin ^{2} \vartheta} \frac{\partial^{2} f}{\partial \varphi^{2}}, \quad \hat{f}=\frac{1}{r} \frac{\partial}{\partial r}(r f) .
$$

The same holds for $\boldsymbol{v}$ with $S$ and $T$ replaced by $\phi$ and $\psi$. In addition, the poloidal and toroidal field potentials are expanded in spherical harmonics

$$
\begin{aligned}
(S, T) & =\sum_{l ; m \neq 0}\left(S_{l}^{m}(r), T_{l}^{m}(r)\right) Y_{l}^{m}(\vartheta, \varphi), \\
S_{l}^{m *} & =S_{l}^{-m}, \quad T_{l}^{m *}=T_{l}^{-m}, \quad Y_{l}^{m}(\vartheta, \varphi)=P_{l}^{|m|}(\cos \vartheta) \exp (\mathrm{i} m \varphi),
\end{aligned}
$$

with corresponding relations for $\phi$ and $\psi$.

Then, $\mathcal{E}=\overline{\boldsymbol{v} \times \boldsymbol{b}}$ can be expressed as a function of the $\phi_{l}^{m}, \psi_{l}^{m}, S_{l}^{m}$ and $T_{l}^{m}$ and a straightforward calculation gives

$$
\begin{aligned}
\mathcal{E}_{r}= & -2 \sum_{l, l^{\prime} ; m>0}\left[\operatorname{Re}\left(\hat{\phi}_{l^{\prime}}^{m *} T_{l}^{m}-\psi_{l^{\prime}}^{m *} \hat{S}_{l}^{m}\right) R_{l^{\prime} l}^{m}\right. \\
& \left.+m \operatorname{Im}\left(\hat{\phi}_{l^{\prime}}^{m *} \hat{S}_{l}^{m}+\psi_{l^{\prime}}^{m *} T_{l}^{m}\right)\left(Q_{l^{\prime} l}^{m}+Q_{l l^{\prime}}^{m}\right) / \sin \vartheta\right] \\
\mathcal{E}_{\vartheta}= & +\frac{2}{r} \sum_{l, l^{\prime} ; m>0}\left[l^{\prime}\left(l^{\prime}+1\right) \operatorname{Re}\left(\phi_{l^{\prime}}^{m *} T_{l}^{m}\right) Q_{l^{\prime} l}^{m}-l(l+1) \operatorname{Re}\left(\psi_{l^{\prime}}^{m *} S_{l}^{m}\right) Q_{l l^{\prime}}^{m}\right. \\
& \left.+m\left(l(l+1) \operatorname{Im}\left(\hat{\phi}_{l^{\prime}}^{m *} S_{l}^{m}\right)+l^{\prime}\left(l^{\prime}+1\right) \operatorname{Im}\left(\phi_{l^{\prime}}^{m *} \hat{S}_{l}^{m}\right)\right) P_{l^{\prime} l}^{m} / \sin \vartheta\right] \\
\mathcal{E}_{\varphi}= & -\frac{2}{r} \sum_{l, l^{\prime} ; m>0}\left[l(l+1) \operatorname{Re}\left(\hat{\phi}_{l^{\prime}}^{m *} S_{l}^{m}\right) Q_{l l^{\prime}}^{m}-l^{\prime}\left(l^{\prime}+1\right) \operatorname{Re}\left(\phi_{l^{\prime}}^{m *} \hat{S}_{l}^{m}\right) Q_{l^{\prime} l}^{m}\right. \\
& \left.+m\left(l^{\prime}\left(l^{\prime}+1\right) \operatorname{Im}\left(\phi_{l^{\prime}}^{m *} T_{l}^{m}\right)+l(l+1) \operatorname{Im}\left(\psi_{l^{\prime}}^{m *} S_{l}^{m}\right)\right) P_{l^{\prime} l}^{m} / \sin \vartheta\right]
\end{aligned}
$$

Here, $\mathcal{E}$ depends on $r$ via $\phi, \psi, S, T, \cdots$, and on $\vartheta$ via $P_{l^{\prime} l}^{m}, Q_{l^{\prime} l}^{m}$ and $R_{l^{\prime} l}^{m}$, which are defined by

$$
\begin{aligned}
P_{l^{\prime} l}^{m} & =P_{l^{\prime}}^{|m|}(\cos \vartheta) P_{l}^{|m|}(\cos \vartheta) \\
Q_{l^{\prime} l}^{m} & =P_{l^{\prime}}^{|m|}(\cos \vartheta) \frac{\mathrm{d} P_{l}^{|m|}(\cos \vartheta)}{\mathrm{d} \vartheta} \\
R_{l^{\prime} l}^{m} & =\frac{\mathrm{d} P_{l^{\prime}}^{|m|}(\cos \vartheta)}{\mathrm{d} \vartheta} \frac{\mathrm{d} P_{l}^{|m|}(\cos \vartheta)}{\mathrm{d} \vartheta}+\frac{m^{2}}{\sin ^{2} \vartheta} P_{l^{\prime}}^{|m|}(\cos \vartheta) P_{l}^{|m|}(\cos \vartheta)
\end{aligned}
$$

The $S_{l}^{m}$ and $T_{l}^{m}$ in expressions (4.18-4.20) have to be determined by an integration of equation (4.14) in order to express $\mathcal{E}$ as a function of the scalar velocity potentials $\phi, \psi, \cdots$, and $\bar{B}$ alone.

By means of the poloidal/toroidal decomposition (4.15), the expansion in spherical harmonics (4.17), and a subsequent integration by parts, the radial component of equation (4.14) and the radial component of the curl of (4.14) can be written as

$$
\begin{aligned}
& \frac{1}{r} \frac{\mathrm{d}^{2}}{\mathrm{~d} r^{2}}\left(r S_{l}^{m}\right)-\frac{l(l+1)}{r^{2}} S_{l}^{m}=F_{l}^{m}, \\
& \frac{1}{r} \frac{\mathrm{d}^{2}}{\mathrm{~d} r^{2}}\left(r T_{l}^{m}\right)-\frac{l(l+1)}{r^{2}} T_{l}^{m}=G_{l}^{m}, \quad r_{i}<r<r_{o},
\end{aligned}
$$


where $m \neq 0$, with

$$
\begin{aligned}
F_{l}^{m} & =\frac{(2 l+1)(l-|m|) !}{4 \pi \eta l(l+1)(l+|m|) !} \int(\boldsymbol{v} \times \overline{\boldsymbol{B}}) \cdot\left(\boldsymbol{r} \times \nabla Y_{l}^{m *}\right) \mathrm{d} \Omega \\
G_{l}^{m} & =\frac{(2 l+1)(l-|m|) !}{4 \pi \eta l(l+1)(l+|m|) !} \int(\nabla \times(\boldsymbol{v} \times \overline{\boldsymbol{B}})) \cdot\left(\boldsymbol{r} \times \nabla Y_{l}^{m *}\right) \mathrm{d} \Omega
\end{aligned}
$$

As usual, $\mathrm{d} \Omega$ means $\sin \vartheta \mathrm{d} \vartheta \mathrm{d} \varphi$ and the integration is over all $\vartheta$ and $\varphi$ of the solid angle. The continuation of $\boldsymbol{b}$ as a potential field in outer space requires

$$
\begin{aligned}
& \frac{\mathrm{d} S_{l}^{m}}{\mathrm{~d} r}-\frac{l}{r} S_{l}^{m}=T_{l}^{m}=0 \quad \text { at } \quad r=r_{i} \quad \text { and } \\
& \frac{\mathrm{d} S_{l}^{m}}{\mathrm{~d} r}+\frac{l+1}{r} S_{l}^{m}=T_{l}^{m}=0 \quad \text { at } \quad r=r_{o}
\end{aligned}
$$

A solution of (4.24-4.25) and (4.28-4.29) is then given by

$$
\begin{aligned}
& S_{l}^{m}=-\int_{r_{i}}^{r_{o}} f_{l}\left(r, r^{\prime}\right) F_{l}^{m}\left(r^{\prime}\right) r^{\prime 2} \mathrm{~d} r^{\prime} \\
& T_{l}^{m}=-\int_{r_{i}}^{r_{o}} g_{l}\left(r, r^{\prime}\right) G_{l}^{m}\left(r^{\prime}\right) r^{\prime 2} \mathrm{~d} r^{\prime}
\end{aligned}
$$

with Green's functions $f_{l}$ and $g_{l}$ as defined in Appendix C. Inserting now the representation of $\boldsymbol{v}$ in terms of $\phi_{l}^{m}$ and $\psi_{l}^{m}$ into relation (4.26) and then the result for $F_{l}^{m}$ into the expression for $S_{l}^{m}$ given by (4.30) we arrive at

$$
\begin{aligned}
S_{l}^{m}(r)= & \frac{(2 l+1)(l-|m|) !}{4 \pi \eta l(l+1)(l+|m|) !} \\
& \sum_{l^{\prime}} \int f_{l}\left(r, r^{\prime}\right)\left\{\left[\hat{\phi}_{l^{\prime}}^{m}\left(r^{\prime}\right) R_{l^{\prime} l}^{m}\left(\vartheta^{\prime}\right)\right.\right. \\
& \left.-\mathrm{i} m \psi_{l^{\prime}}^{m}\left(r^{\prime}\right)\left(Q_{l^{\prime} l}^{m}\left(\vartheta^{\prime}\right)+Q_{l^{\prime}}^{m}\left(\vartheta^{\prime}\right)\right) / \sin \vartheta^{\prime}\right] \bar{B}_{r}\left(r^{\prime}, \vartheta^{\prime}\right) \\
& -\frac{l^{\prime}\left(l^{\prime}+1\right)}{r^{\prime}} \phi_{l^{\prime}}^{m}\left(r^{\prime}\right) Q_{l^{\prime} l}^{m}\left(\vartheta^{\prime}\right) \bar{B}_{\vartheta}\left(r^{\prime}, \vartheta^{\prime}\right) \\
& \left.+\mathrm{i} m \frac{l^{\prime}\left(l^{\prime}+1\right)}{r^{\prime}} \phi_{l^{\prime}}^{m}\left(r^{\prime}\right)\left(P_{l^{\prime} l}^{m}\left(\vartheta^{\prime}\right) / \sin \vartheta^{\prime}\right) \bar{B}_{\varphi}\left(r^{\prime}, \vartheta^{\prime}\right)\right\} \mathrm{d} v^{\prime},
\end{aligned}
$$

in which the integration is over the whole fluid shell. When proceeding analogously with relation (4.27), the result for $G_{l}^{m}$ contains at first derivatives of $\bar{B}_{r}, \bar{B}_{\vartheta}$, and $\bar{B}_{\varphi}$ with respect to $r$ and $\vartheta$. We may remove them by means of integration by parts. In this way we find $T_{l}^{m}$ in the form

$$
\begin{aligned}
T_{l}^{m}(r)= & \frac{(2 l+1)(l-|m|) !}{4 \pi \eta l(l+1)(l+|m|) !} \sum_{l^{\prime}} \int\left\{\left(\tilde{g}_{l}\left(r, r^{\prime}\right)\right.\right. \\
& \times\left(\mathrm{i} m \hat{\phi}_{l^{\prime}}^{m}\left(r^{\prime}\right)\left(Q_{l^{\prime} l}^{m}\left(\vartheta^{\prime}\right)+Q_{l l^{\prime}}^{m}\left(\vartheta^{\prime}\right)\right) / \sin \vartheta^{\prime}-\psi_{l^{\prime}}^{m}\left(r^{\prime}\right) R_{l^{\prime} l}^{m}\left(\vartheta^{\prime}\right)\right) \bar{B}_{r}\left(r^{\prime}, \vartheta^{\prime}\right) \\
& -\frac{1}{r^{\prime}}\left[l(l+1) g_{l}\left(r, r^{\prime}\right)\left(\mathrm{i} m \hat{\phi}_{l^{\prime}}^{m}\left(r^{\prime}\right) P_{l^{\prime} l}^{m}\left(\vartheta^{\prime}\right) / \sin \vartheta^{\prime}-\psi_{l^{\prime}}^{m}\left(r^{\prime}\right) Q_{l l^{\prime}}^{m}\left(\vartheta^{\prime}\right)\right)\right. \\
& \left.+\mathrm{i} m l^{\prime}\left(l^{\prime}+1\right) \tilde{g}_{l}\left(r, r^{\prime}\right) \phi_{l^{\prime}}^{m}\left(r^{\prime}\right) P_{l^{\prime} l}^{m}\left(\vartheta^{\prime}\right) / \sin \vartheta^{\prime}\right] \bar{B}_{\vartheta}\left(r^{\prime}, \vartheta^{\prime}\right) \\
& +\frac{1}{r^{\prime}}\left[l(l+1) g_{l}\left(r, r^{\prime}\right)\left(\hat{\phi}_{l^{\prime}}^{m}\left(r^{\prime}\right) Q_{l l^{\prime}}^{m}\left(\vartheta^{\prime}\right)+\mathrm{i} m \psi_{l^{\prime}}^{m}\left(r^{\prime}\right) P_{l l^{\prime}}^{m}\left(\vartheta^{\prime}\right) / \sin \vartheta^{\prime}\right)\right. \\
& \left.\left.-l^{\prime}\left(l^{\prime}+1\right) \tilde{g}_{l}\left(r, r^{\prime}\right) \phi_{l^{\prime}}^{m}\left(r^{\prime}\right) Q_{l^{\prime} l}^{m}\left(\vartheta^{\prime}\right)\right] \bar{B}_{\varphi}\left(r^{\prime}, \vartheta^{\prime}\right)\right\} \mathrm{d} v^{\prime}
\end{aligned}
$$


with

$$
\tilde{g}_{l}\left(r, r^{\prime}\right)=\frac{1}{r^{\prime}} \frac{\partial}{\partial r^{\prime}}\left(r^{\prime} g_{l}\left(r, r^{\prime}\right)\right)
$$

Now, we may write

$$
\mathcal{E}_{\kappa}(r, \vartheta)=\int K_{\kappa \lambda}\left(r, \vartheta ; r^{\prime}, \vartheta^{\prime}\right) \bar{B}_{\lambda}\left(r^{\prime}, \vartheta^{\prime}\right) \mathrm{d} v^{\prime},
$$

with some kernel $K_{\kappa \lambda}$. Here indices like $\kappa$ or $\lambda$ are used for $r, \vartheta$ or $\varphi$, and the summation convention is adopted. The integration is again over the whole fluid shell. The $K_{\kappa \lambda}$ can then be concluded from relations (4.18-4.20), (4.32) and (4.33).

As an example, we have

$$
\begin{aligned}
K_{r r}\left(r, \vartheta ; r^{\prime}, \vartheta^{\prime}\right)= & \frac{1}{2 \pi \eta} \sum_{l, l^{\prime}, l^{\prime \prime} ; m>0} \frac{(2 l+1)(l-|m|) !}{l(l+1)(l+|m|) !} \\
& \times\left\{\left[\hat{f}_{l}\left(r, r^{\prime}\right) \operatorname{Im}\left(\psi_{l^{\prime}}^{m *}(r) \psi_{l^{\prime \prime}}^{m}\left(r^{\prime}\right)\right)+\tilde{g}_{l}\left(r, r^{\prime}\right) \operatorname{Im}\left(\hat{\phi}_{l^{\prime}}^{m *}(r) \hat{\phi}_{l^{\prime \prime}}^{m}\left(r^{\prime}\right)\right)\right]\right. \\
& \times m R_{l^{\prime} l}^{m}(\vartheta)\left(Q_{l^{\prime \prime} l}^{m}\left(\vartheta^{\prime}\right)+Q_{l l^{\prime \prime}}^{m}\left(\vartheta^{\prime}\right)\right) / \sin \vartheta^{\prime} \\
& +\left[\hat{f}_{l}\left(r, r^{\prime}\right) \operatorname{Re}\left(\hat{\phi}_{l^{\prime}}^{m *}(r) \psi_{l^{\prime \prime}}^{m}\left(r^{\prime}\right)\right)-\tilde{g}_{l}\left(r, r^{\prime}\right) \operatorname{Re}\left(\psi_{l^{\prime}}^{m *}(r) \hat{\phi}_{l^{\prime \prime}}^{m}\left(r^{\prime}\right)\right)\right] \\
& \times m^{2}\left(\left(Q_{l^{\prime} l}^{m}(\vartheta)+Q_{l l^{\prime}}^{m}(\vartheta)\right) / \sin \vartheta\right)\left(Q_{l^{\prime \prime} l}^{m}\left(\vartheta^{\prime}\right)+Q_{l l^{\prime \prime}}^{m}\left(\vartheta^{\prime}\right)\right) / \sin \vartheta^{\prime} \\
& +\left[\hat{f}_{l}\left(r, r^{\prime}\right) \operatorname{Re}\left(\psi_{l^{\prime}}^{m *}(r) \hat{\phi}_{l^{\prime \prime}}^{m}\left(r^{\prime}\right)\right)+\tilde{g}_{l}\left(r, r^{\prime}\right) \operatorname{Re}\left(\hat{\phi}_{l^{\prime}}^{m *}(r) \psi_{l^{\prime \prime}}^{m}\left(r^{\prime}\right)\right)\right] \\
& \times R_{l^{\prime} l}^{m}(\vartheta) R_{l^{\prime \prime} l}^{m}\left(\vartheta^{\prime}\right) \\
& -\left[\hat{f}_{l}\left(r, r^{\prime}\right) \operatorname{Im}\left(\hat{\phi}_{l^{\prime}}^{m *}(r) \hat{\phi}_{l^{\prime \prime}}^{m}\left(r^{\prime}\right)-\tilde{g}_{l}\left(r, r^{\prime}\right) \operatorname{Im}\left(\psi_{l^{\prime}}^{m *}(r) \psi_{l^{\prime \prime}}^{m}\left(r^{\prime}\right)\right)\right]\right. \\
& \left.\times m\left(\left(Q_{l^{\prime} l}^{m}(\vartheta)+Q_{l l^{\prime}}^{m}(\vartheta)\right) / \sin \vartheta\right) R_{l^{\prime \prime l} l}^{m}\left(\vartheta^{\prime}\right)\right\}
\end{aligned}
$$

in which $\hat{f}_{l}\left(r, r^{\prime}\right)=1 / r \partial\left(r f_{l}\left(r, r^{\prime}\right)\right) / \partial r$.

The component $\tilde{a}_{r r}$ in the sense of $\mathcal{E}_{\kappa}=\tilde{a}_{\kappa \lambda} \bar{B}_{\lambda}+\tilde{b}_{\kappa \lambda r} \partial \bar{B}_{\lambda} / \partial r+\cdots$ is then given by

$$
\tilde{a}_{r r}(r, \vartheta)=\int K_{r r}\left(r, \vartheta ; r^{\prime}, \vartheta^{\prime}\right) \mathrm{d} v^{\prime}
$$

again with the integration over the fluid shell (see also Section 3.1). Making use of the orthogonality relations in Appendix C, we finally obtain

$$
\begin{aligned}
\tilde{a}_{r r}(r, \vartheta)= & \frac{2}{\eta} \sum_{l, l^{\prime} ; m>0}\left\{\int _ { 0 } ^ { R } \left(\hat{f}_{l}\left(r, r^{\prime}\right) \operatorname{Re}\left(\psi_{l^{\prime}}^{m *}(r) \hat{\phi}_{l}^{m}\left(r^{\prime}\right)\right)\right.\right. \\
& \left.+\tilde{g}_{l}\left(r, r^{\prime}\right) \operatorname{Re}\left(\hat{\phi}_{l^{\prime}}^{m *}(r) \psi_{l}^{m}\left(r^{\prime}\right)\right)\right) r^{\prime 2} \mathrm{~d} r^{\prime} R_{l^{\prime} l}^{m}(\vartheta) \\
& -\int_{0}^{R}\left(\hat{f}_{l}\left(r, r^{\prime}\right) \operatorname{Im}\left(\hat{\phi}_{l^{\prime}}^{m *}(r) \hat{\phi}_{l}^{m}\left(r^{\prime}\right)\right)-\tilde{g}_{l}\left(r, r^{\prime}\right) \operatorname{Im}\left(\psi_{l^{\prime}}^{m *}(r) \psi_{l}^{m}\left(r^{\prime}\right)\right)\right) r^{\prime 2} \mathrm{~d} r^{\prime} \\
& \left.\times m\left(Q_{l^{\prime} l}^{m}(\vartheta)+Q_{l l^{\prime}}^{m}(\vartheta)\right) / \sin \vartheta\right\}
\end{aligned}
$$

Relation (4.38) provides the $\tilde{a}_{r r}$ component for a given velocity field represented by $\phi$ and $\psi$. All the other components are derived similarly and are given in Appendix D. 



\section{Mean-field coefficients: results}

The methods derived in the previous chapter have been applied to four different velocity fields which originate from a simulation of rotating magnetoconvection, from the modelling of a simple (quasi-)stationary dynamo, and from two time dependent dynamos of which one exhibits polarity reversals. According to their actual features, the examples examined here will be used to investigate the $\alpha$-quenching in consequence of the presence of a mean-field (Section 5.1.4), the deviations from the isotropic and SOCA-approximation (Section 5.1.3 and Section 5.2.4) as well as the time variability of mean-field coefficients (Section 5.3 and Section 5.4).

\subsection{Simulation of rotating magnetoconvection}

\subsubsection{Velocity and magnetic field}

The first example considered here is adopted from Olson et al. (1999) with the governing parameters $E=3 \times 10^{-4}, R a=94\left(=1.5 R a_{c}\right)$, and $P r=P m=1$. Moreover, an axisymmetric toroidal magnetic field is imposed via inhomogeneous toroidal boundary conditions of the form

$$
B_{\varphi}=\Lambda_{o} \sin (2 \vartheta) \quad \text { at } r=r_{i}, \quad r=r_{o}
$$

in which $\Lambda_{o}$ is the Elsasser number based on the amplitude $B_{o}$ of the imposed field

$$
\Lambda_{o}=\frac{B_{o}^{2}}{\varrho \mu \eta \Omega}
$$

Figure 5.1 shows the radial velocity field for $\Lambda_{o}=1$ at $r=0.59 r_{o}$. Here, upflows are coloured red while downflows are blue and a typical columnar convection pattern is revealed. Superposed on the columnar convection is a secondary flow parallel or antiparallel to the polar axis. Altogether, this flow pattern produces a helicity distribution as presented in Figure 5.2 in which negative helicity in the northern and positive helicity in the southern hemisphere appears to be a basic property of columnar convection in a rotating spherical shell. The resulting magnetic Reynolds number is about 12 and far too low for the onset of self-sustaining dynamo action. Nevertheless, fundamental dynamo processes related to this convection pattern are present and can be analysed by means of mean-field theory; so for example the generation of poloidal out of toroidal field, which results in a radial component of the magnetic field as displayed in Figure 5.3.

Except for a steady azimuthal drift of the convection columns with a drift frequency $\omega=-5.46 \nu / D^{2}$, the flow and thus the kinetic and also the magnetic energy are steady. 


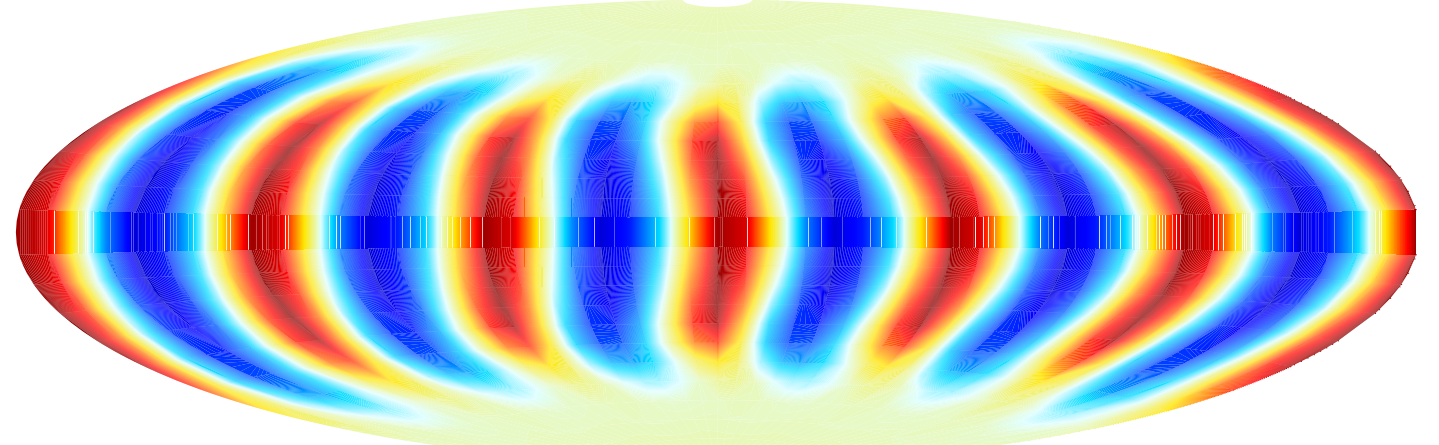

Figure 5.1: The radial velocity in the magnetoconvection example with $\Lambda_{o}=1$ and assumed 6-fold azimuthal symmetry at $r=0.59 r_{o}$, normalised by its absolute maximum given by $V_{r}=16.98 \nu / D$. In the colour coding, dark blue and dark red correspond to -1 and +1 , respectively, and the contour stepping to \pm 0.1 . All following contour plots will be presented in the same style.

However, this time dependency can be removed by a transformation to a corotating frame of reference. This has been done by prescribing a mean velocity $\bar{V}_{\varphi}=\omega r \sin \vartheta$ at the inner and outer boundary in difference from the no-slip boundary conditions originally assumed. The related toroidal velocity potential is then determined by

$$
-\frac{1}{r} \frac{\partial z}{\partial \vartheta}=\omega r \sin \vartheta \quad \text { at } r=r_{i}, \quad r=r_{o}
$$
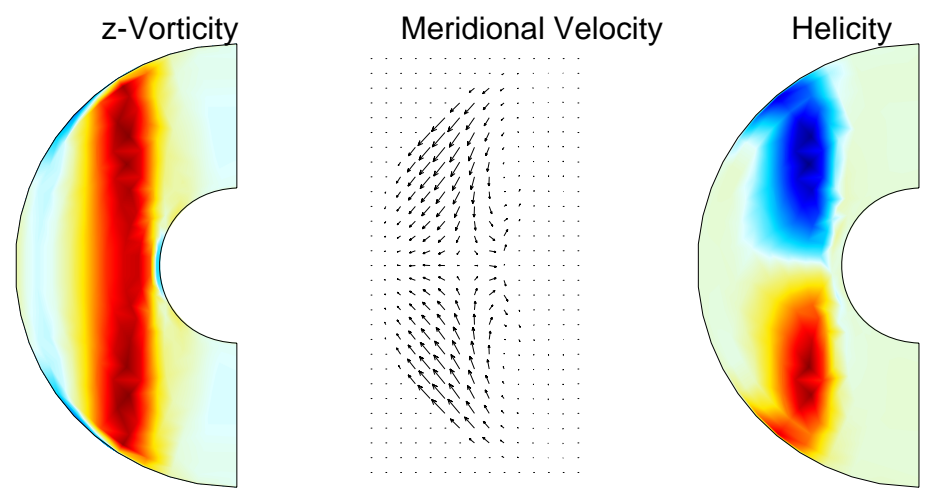

Figure 5.2: Meridional slices at a certain longitude. The primary columnar flow with positive z-vorticity together with a secondary meridional flow with a negative axial component in the northern and a positive in the southern hemisphere give rise to negative helicity in the northern and positive in the southern hemisphere. 


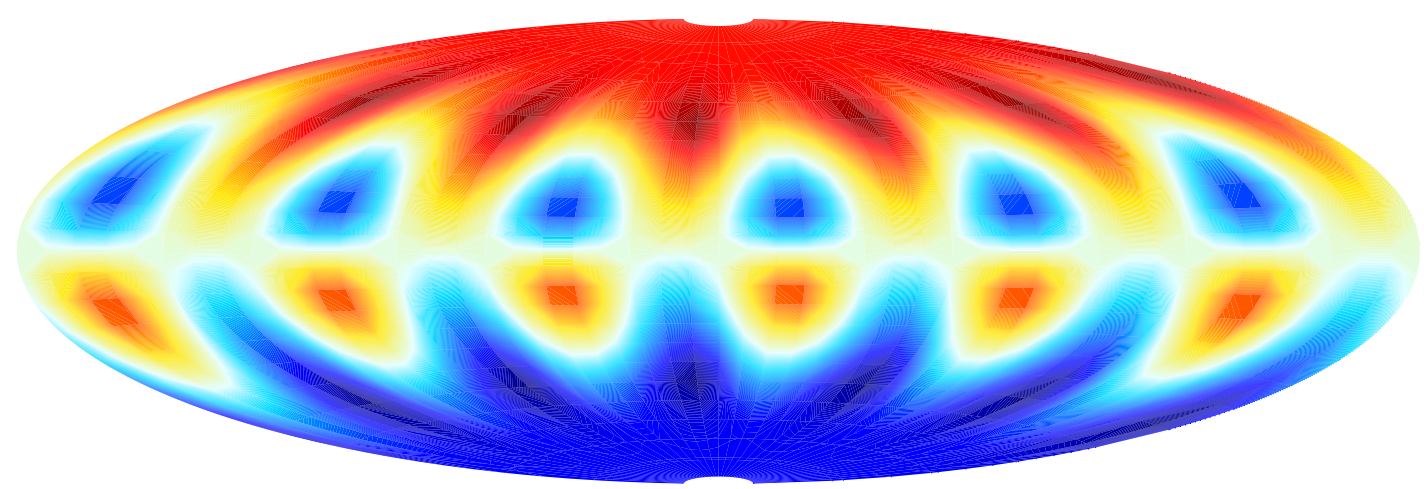

Figure 5.3: The radial magnetic field in the magnetoconvection example at $r=0.59 r_{o}$. Its maximum is given by $B_{r}=0.64(\varrho \mu \eta \Omega)^{1 / 2}$, and the contour levels are chosen as in Figure 5.1.

which is easily integrated to yield

$$
z=\cos \vartheta \omega r^{2}=\sqrt{\frac{4 \pi}{3}} Y_{1}^{0} \omega r^{2} \quad \text { at } r=r_{i}, \quad r=r_{o}
$$

Note that except for terms of order $|\Omega \times \boldsymbol{r}| / c$ Maxwell's equations remain invariant under a transformation to a rotating frame of reference with angular velocity $\Omega$. Thus, $|\boldsymbol{\Omega} \times \boldsymbol{r}| / c \ll 1$, in addition to $|\boldsymbol{v}| / c \ll 1$, has been already presumed for equation (2.4) to hold. By the same token, $|(\Omega+\boldsymbol{\omega}) \times \boldsymbol{r}| / c \ll 1$ may be assumed, and still the induction equation remains unchanged.

\subsubsection{Non-covariant and covariant mean-field coefficients}

Non-covariant and covariant ${ }^{1}$ mean-field coefficients corresponding to the example of magnetoconvection are presented in Figure 5.4 and Figure 5.5, respectively. A comparison between both reveals only minor differences in the amplitudes of some components $\tilde{a}_{\kappa \lambda}$ and $a_{\kappa \lambda}$. This reduces the necessity to distinguish between them, and statements about their principal properties apply equally to both.

The results for the $\tilde{a}_{\kappa \lambda}$ obtained by the two methods explained in the previous chapter, (I) and (II), do not completely coincide. This was to be expected, because approach (II) is based on the second order correlation approximation. In the steady case considered here, it is justified for $R m \ll 1$, and consequently, both results come into agreement, if $R m$ is scaled down (see also Appendix E). This test provides insight into the scope of SOCA and at the same time confirms the reliability of approach (I), which has been used to derive the mean-field coefficients presented in this chapter.

All mean-field coefficients are closely related to the columnar convection, which takes place outside the inner core tangent cylinder. In consequence of the symmetry properties

\footnotetext{
${ }^{1}$ Linguistic convention: covariant mean-field coefficients are mean-field coefficients which originate from an expansion of $\mathcal{E}$ which is form-invariant under coordinate transformations.
} 

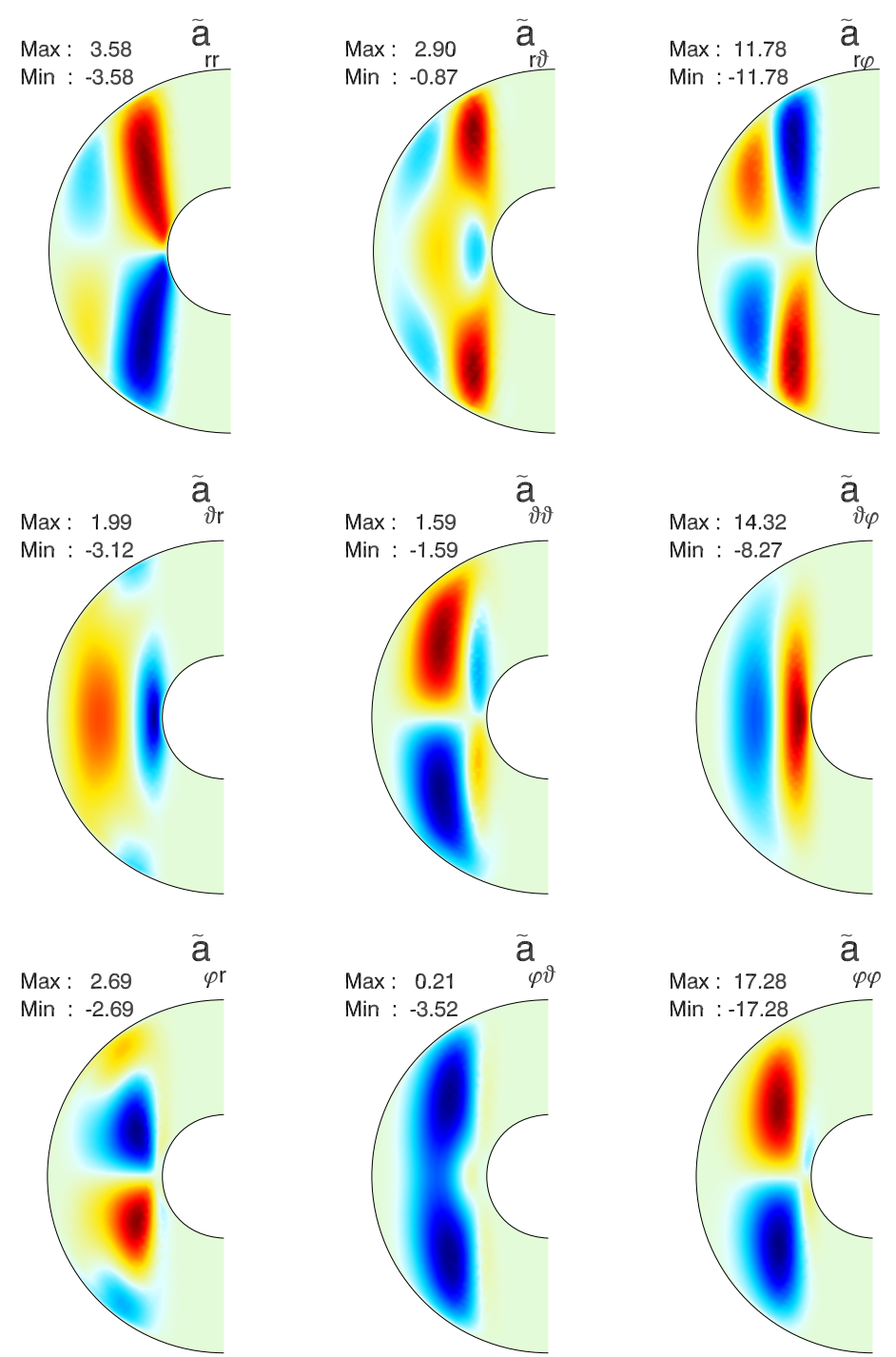

Figure 5.4: Non-covariant mean-field coefficients in the meridional plane in the magnetoconvection example, determined by method (I) in units of $\nu / D$. For each component, the colour coding is separately adjusted to its maximum or, having a larger modulus, to its minimum.

of the velocity field and of the induction equation all components of $\boldsymbol{a}$ are either symmetric or antisymmetric with respect to the equator, as predicted in Section 3.4. None of the mean-field coefficients vanishes. For their interpretation it is instructive to regard the expansion of $\mathcal{E}$ for a diagonal/non-diagonal decomposition of the tensor $\boldsymbol{a}$ :

$$
\begin{aligned}
\mathcal{E} & =\boldsymbol{a} \overline{\boldsymbol{B}}+\boldsymbol{b} \nabla \overline{\boldsymbol{B}} \\
& =\hat{\boldsymbol{\alpha}} \overline{\boldsymbol{B}}+\gamma_{D} \times \overline{\boldsymbol{B}}_{\mathrm{pol}}+\gamma_{M T} \times \overline{\boldsymbol{B}}_{\mathrm{tor}}+\gamma_{M P} \times \overline{\boldsymbol{B}}_{\mathrm{pol}}+\boldsymbol{b} \nabla \overline{\boldsymbol{B}}
\end{aligned}
$$

Here, all higher than first order derivatives of $\bar{B}$ have been omitted and the following 

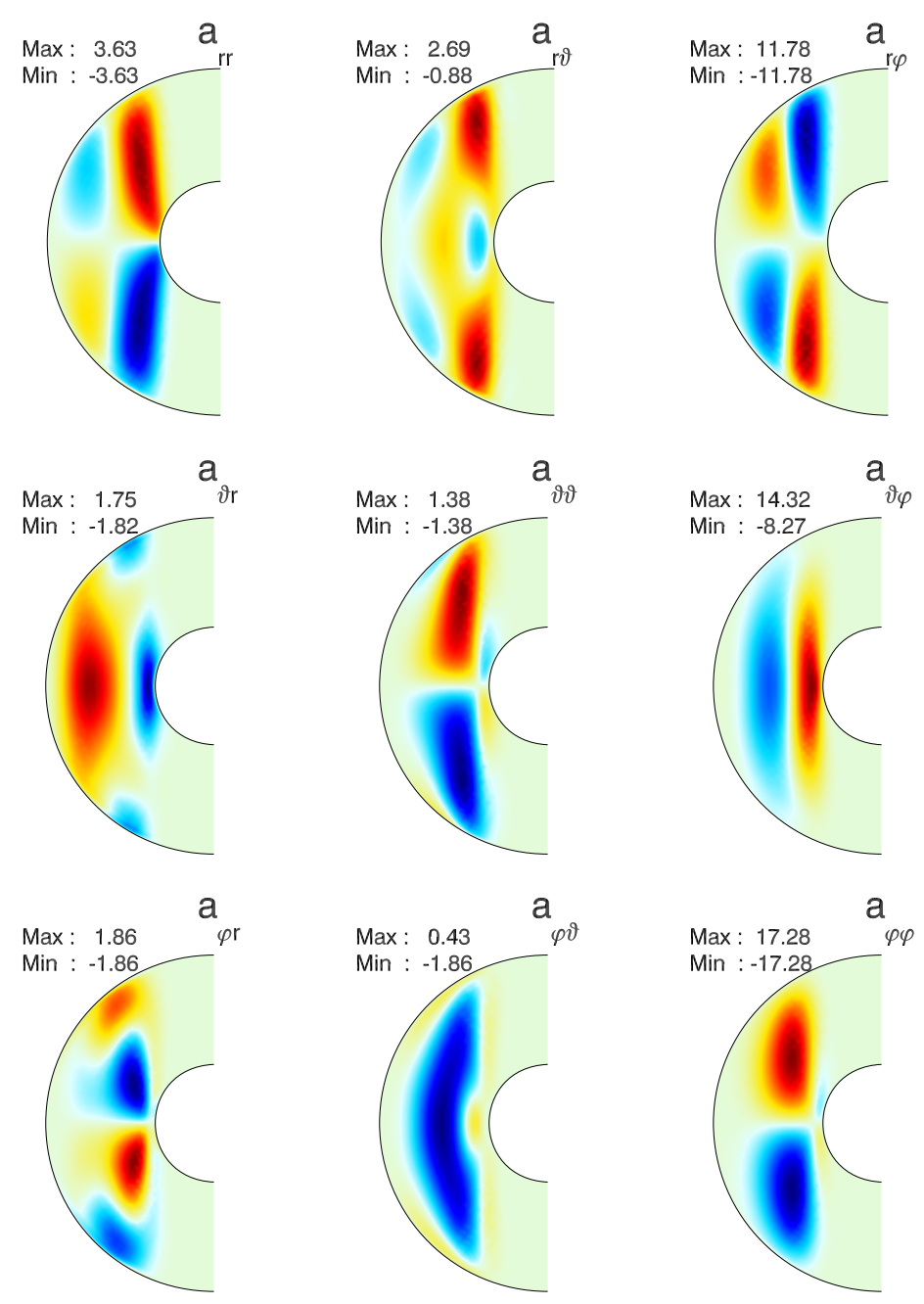

Figure 5.5: Components of the tensor $\boldsymbol{a}$ in the meridional plane in the magnetoconvection example, determined by method (I) in units of $\nu / D$. The components $a_{\kappa \lambda}$ exhibit only minor differences in comparison to the corresponding set of non-covariant mean-field coefficients, see Figure 5.4

notations have been used:

$$
\begin{gathered}
\hat{\boldsymbol{\alpha}}=\left(\begin{array}{ccc}
a_{r r} & \left(a_{r \vartheta}+a_{\vartheta r}\right) / 2 & 0 \\
\left(a_{\vartheta r}+a_{r \vartheta}\right) / 2 & a_{\vartheta \vartheta} & 0 \\
0 & 0 & a_{\varphi \varphi}
\end{array}\right), \\
\gamma_{D}=\left(\begin{array}{c}
0 \\
0 \\
\left(a_{\vartheta r}-a_{r \vartheta}\right) / 2
\end{array}\right), \quad \gamma_{M T}=\left(\begin{array}{c}
-a_{\vartheta \varphi} \\
a_{r \varphi} \\
0
\end{array}\right), \quad \gamma_{M P}=\left(\begin{array}{c}
a_{\varphi \vartheta} \\
-a_{\varphi r} \\
0
\end{array}\right)
\end{gathered}
$$


Furthermore, $\overline{\boldsymbol{B}}$ is separated in its poloidal and toroidal parts

$$
\overline{\boldsymbol{B}}_{\mathrm{pol}}=\left(\begin{array}{c}
\bar{B}_{r} \\
\bar{B}_{\vartheta} \\
0
\end{array}\right), \quad \overline{\boldsymbol{B}}_{\mathrm{tor}}=\left(\begin{array}{c}
0 \\
0 \\
\bar{B}_{\varphi}
\end{array}\right)
$$

All components contributing to the symmetric matrix $\hat{\boldsymbol{\alpha}}$ act as inductive terms. This applies in particular to the diagonal components of $\boldsymbol{a}$ among which $a_{\varphi \varphi}$ dominates, indicating that the generation of poloidal field out of toroidal is much more effective than vice versa. But, due to the non-vanishing components $a_{r r}$ and $a_{\vartheta \vartheta}$, generation of toroidal field by an $\alpha$-effect also takes place.

Except for the term $\left(a_{\vartheta r}+a_{r \vartheta}\right) / 2$, the non-diagonal components contribute only to the vectors $\gamma_{D}, \gamma_{M T}$, and $\gamma_{M P}$. While the vector $\gamma$, introduced in (3.25), can be interpreted as an advective velocity which acts equally on all components of $\overline{\boldsymbol{B}}$, the representation (5.5) aims to distinguish the action of the non-diagonal components on the poloidal and toroidal mean field. Following this line, $\gamma_{M T}$ and $\gamma_{M P}$ are interpreted formally as a contribution to the mean meridional flow advecting the mean toroidal and poloidal field, respectively. Moreover, $\gamma_{D}$ can be added to a mean toroidal velocity and therefore influences the generation of toroidal field by differential rotation.

For the sake of completeness, it should be noted, that unlike in ideal MHD, it is not possible to define a moving surface in which flux is conserved. But still, it is possible to define a velocity that optimises flux-conservation for each divergence-free part of the field (Kichatinov 1991, Ossendrijver et al. 2002). Whenever referring to advection processes in this work, advection has to be understood in this sense.

By far most dominant among the non-diagonal components are $a_{r \varphi}$ and $a_{\vartheta \varphi}$ constituting $\gamma_{M T}$. A vector plot of $\gamma_{M T}$ is drawn in Figure 5.6. It indicates that the mean toroidal field is advected towards the equatorial plane in upwellings near the outer boundary while it is advected towards higher latitudes in downflows in deeper layers. Following Olson et al. (1999), this characteristic can be attributed to the columnar convection and the spherical boundaries. A material line of fluid that is transported in an upwelling towards the outer boundary has to shorten, and this causes a convergent flow towards the equatorial plane which is independent of the secondary, helicity-producing flow component. The opposite is true for the downwellings. We will see in the following that the dominating non-diagonal components $a_{r \varphi}$ and $a_{\vartheta \varphi}$ have a major effect on the resulting field topology.

In Figure 5.7, the diffusion tensor is shown. It is given by

$$
D_{\kappa \lambda}=P m^{-1} \delta_{\kappa \lambda}+\beta_{\kappa \lambda}
$$

at which the molecular diffusion has been taken into account. Although the molecular diffusivity is rather high $(P m=1)$, and the vigour of the convection is rather low $(R a=$ $1.5 R a_{\text {crit }}$ ), the turbulent diffusion dominates already over the molecular one in the region where convection takes place. In this example, the diffusion tensor proves to be positive definite everywhere in the meridional plane. In order to show this, it is sufficient to note that all diagonal components of $\boldsymbol{D}$ are positive, and each sub-determinant is positive as well. The physical meaning of $\boldsymbol{\beta}$ being positive definite is best illustrated considering the 


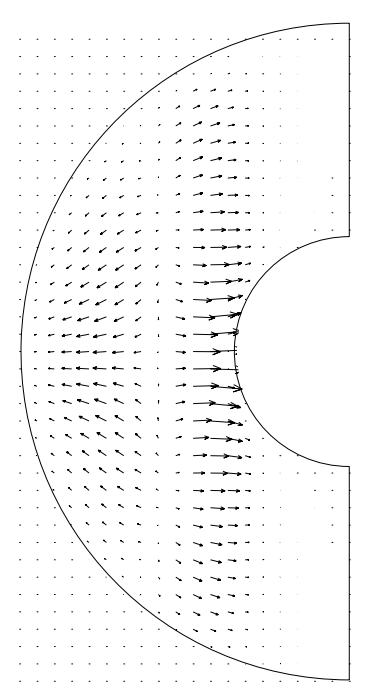

Figure 5.6: Vector plot of $\gamma_{\mathrm{MT}}$ in the meridional plane. The vector $\gamma_{\mathrm{MT}}$ is interpreted as mean meridional velocity advecting the mean toroidal field towards the equatorial plane near the outer boundary while the field is advected towards the inner core tangent cylinder and towards higher latitudes in deeper layers.

energy balance

$$
\frac{d}{d t} \int_{\infty} \frac{\overline{\boldsymbol{B}}^{2}}{2} d v=-\int_{\mathcal{V}} \overline{\boldsymbol{j}} \cdot \overline{\boldsymbol{E}} d v
$$

with $\bar{E}$ being the mean electrical field, $\bar{j}$ the mean current density and $\mathcal{V}$ the volume of the considered fluid shell. Furthermore, we assume

$$
\overline{\boldsymbol{j}}=\operatorname{Pm}(\overline{\boldsymbol{E}}+\overline{\boldsymbol{V}} \times \overline{\boldsymbol{B}}+\mathcal{E})
$$

and

$$
\mathcal{E}=-\boldsymbol{\alpha} \overline{\boldsymbol{B}}-\boldsymbol{\gamma} \times \overline{\boldsymbol{B}}-\boldsymbol{\beta}(\nabla \times \overline{\boldsymbol{B}})-\boldsymbol{\delta} \times(\nabla \times \overline{\boldsymbol{B}})-\boldsymbol{\kappa}(\nabla \overline{\boldsymbol{B}})^{(\mathrm{sy})}+\mathcal{E}^{(>1)}
$$

in which the additional term $\mathcal{E}^{(>1)}$ represents parts of $\mathcal{E}$ proportional to higher than first order derivatives of $\overline{\boldsymbol{B}}$. Then, it follows

$$
\begin{aligned}
\overline{\boldsymbol{j}} \cdot \overline{\boldsymbol{E}}= & \left(P m^{-1} \delta_{\kappa \lambda}+\beta_{\kappa \lambda}\right) \bar{j}_{\kappa} \bar{j}_{\lambda}+\alpha_{\kappa \lambda} \bar{j}_{\kappa} \bar{B}_{\lambda} \\
& +(\overline{\boldsymbol{V}}-\gamma) \cdot(\overline{\boldsymbol{j}} \times \overline{\boldsymbol{B}})+\overline{\boldsymbol{j}} \cdot\left(\boldsymbol{\kappa}(\nabla \overline{\boldsymbol{B}})^{(\mathrm{sy})}\right)-\overline{\boldsymbol{j}} \cdot \mathcal{E}^{(>1)}
\end{aligned}
$$

If $P m^{-1} \delta_{\kappa \lambda}+\beta_{\kappa \lambda}$ is positive definite, the mean magnetic field can not grow in the absence of an inductive action of $\boldsymbol{\alpha}, \overline{\boldsymbol{V}}, \boldsymbol{\gamma}, \boldsymbol{\kappa}$, and $\mathcal{E}^{(>1)}$. On the other hand, the tensor $\mathrm{Pm}^{-1} \delta_{\kappa \lambda}+$ $\beta_{\kappa \lambda}$ could initiate an energy supply to the mean magnetic field, if it were not positive definite and not balanced by further terms. Note that the term $\delta \times(\nabla \times \bar{B})$ in (5.12) does not contribute to the integral $\int_{\mathcal{V}} \overline{\boldsymbol{j}} \cdot \overline{\boldsymbol{E}} d v$. In consequence, it is not possible to drive a dynamo by the $\delta \times j$-effect alone. 

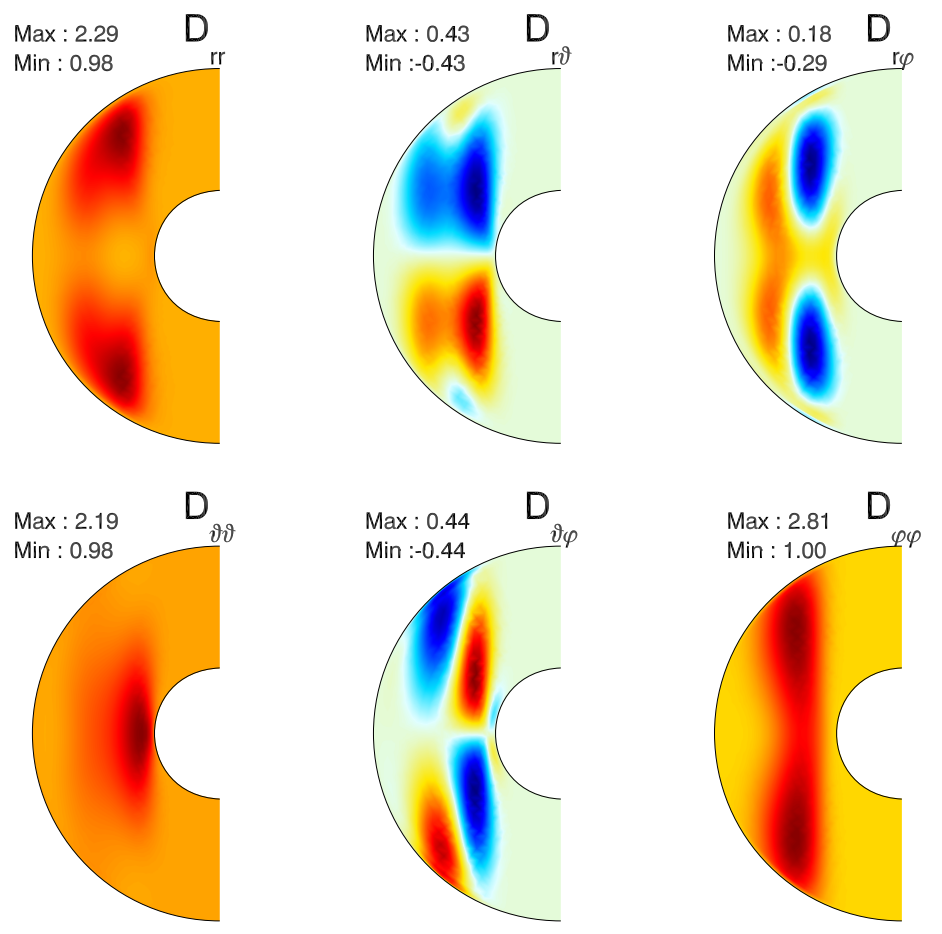

Figure 5.7: The symmetric diffusion tensor in the magnetoconvection example in the meridional plane, determined by method (I) in units of $\nu$. The turbulent diffusion exceeds the molecular one in regions where convection takes place.

\subsubsection{Isotropic approximation}

A scalar approximation for $\boldsymbol{\alpha}$ related to the example considered here, $\alpha_{I}$, is given by relation (3.43). In Figure 5.8, $\alpha_{I}$ is plotted in comparison with $-\alpha_{\varphi \varphi}$ derived by method (I) and (II). The components displayed are quite similar in shape, and their amplitudes show differences of less than 20\%. Although $\alpha_{\varphi \varphi}$ represents the isotropic part of the $\alpha$ tensor, as has been demostrated in Section 3.4, it does not coincide exactly with $\alpha_{I}$. This is not surprising because several approximations required to derive (3.43) are not completely justified, e.g the assumption of $\boldsymbol{v}$ being statistically isotropic and homogeneous, or the neglect of the mean flow. There are also differences between $\alpha_{\varphi \varphi}$, obtained by approach (I), and $\alpha_{\varphi \varphi}$ in the second order correlation approximation, as given by approach (II). Even though these coefficients agree perfectly in shape, the second order correlation approximation leads typically to overestimated amplitudes.

\subsection{4 $\alpha$ - and $\beta$-quenching}

There is a debate going on whether the $\alpha$-tensor is catastrophically quenched due to the back reaction of the Lorentz force. Vainshtein and Cattaneo (1992), Cattaneo and Hughes (1996), and in a series of papers Gruzinov and Diamond (1994, 1995, 1996) argue that the amplitudes of the $\alpha$-components are quenched to a very small value before the magnetic 

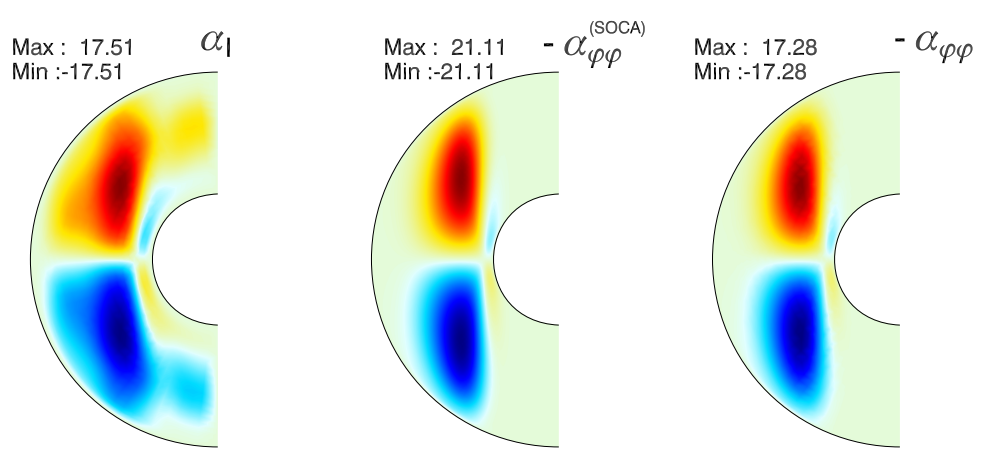

Figure 5.8: From left to right: $\alpha$ due to the isotropic approximation (3.43), $-\alpha_{\varphi \varphi}$ in the second order correlation approximation derived by approach (II) and $-\alpha_{\varphi \varphi}$ determined by method (I). Maxima and minima are given in scales of $\nu / D$.

field reaches its equipartition value, $B_{\text {eq }}^{2}=\mu \varrho V_{\text {rms }}^{2}$. They suggest a scaling law

$$
\alpha=\frac{\alpha_{0}}{1+R m \overline{\boldsymbol{B}}^{2} / B_{\mathrm{eq}}^{2}}
$$

while traditionally

$$
\alpha=\frac{\alpha_{0}}{1+\overline{\boldsymbol{B}}^{2} / B_{\text {eq }}^{2}}
$$

has been assumed (Rüdiger 1973) to scale the kinematic value of $\alpha$, here denoted $\alpha_{0}$, in the nonlinear regime. More specific scaling laws which account for the quenching properties of each diagonal component of the $\alpha$-tensor separately are given by Rüdiger and Kichatinov (1993). In consequence of (5.14), typically flows with large $R m$ prevalent in most astrophysical circumstances would be incapable of generating sizable magnetic fields by slow dynamo action.

In the context of the dynamo simulations considered here, relation (5.14) could not be verified. This had to be expected because most simulations for the geodynamo operate in the strong field regime with the magnetic energy density exceeding the kinetic energy density in most examples (Christensen et al. 1999). The applicability of (5.14) has been tested carrying out a number of simulations with $\Lambda_{0}$ in (5.1) varying in a range between 0 and 2. Subsequently, for each of them mean-field coefficients have been determined. Figure 5.9 shows the r.m.s. value of $\alpha_{\varphi \varphi}$ normalised by $V_{\text {rms }}$ in dependence of $\Lambda$. In addition, the non-axisymmetric kinetic energy density (dashed line) and the axisymmetric kinetic energy density (dotted line) are displayed. Both have been rescaled by a factor of 300 for presentation reasons. Contrary to (5.14) and (5.15), $\left(\alpha_{\varphi \varphi}\right)_{\text {rms }}$ grows at first in presence of a magnetic field. It reaches its maximum value at $\Lambda=0.3$ corresponding to $(\overline{\boldsymbol{B}})_{\mathrm{rms}} \approx 2.8 B_{\text {eq }}$. This is consistent with the incline of the non-axisymmetric kinetic energy density, i.e. with the increasing vigour of convection. Although strong magnetic fields are usually known to inhibit convection because additional work has to be done against the Lorentz force, the presence of a magnetic field may also stimulate convection as demonstrated in the example under consideration. This is explained taking the inhibiting effect due to rotation into account: Convection in a sphere must be z-dependent, which 


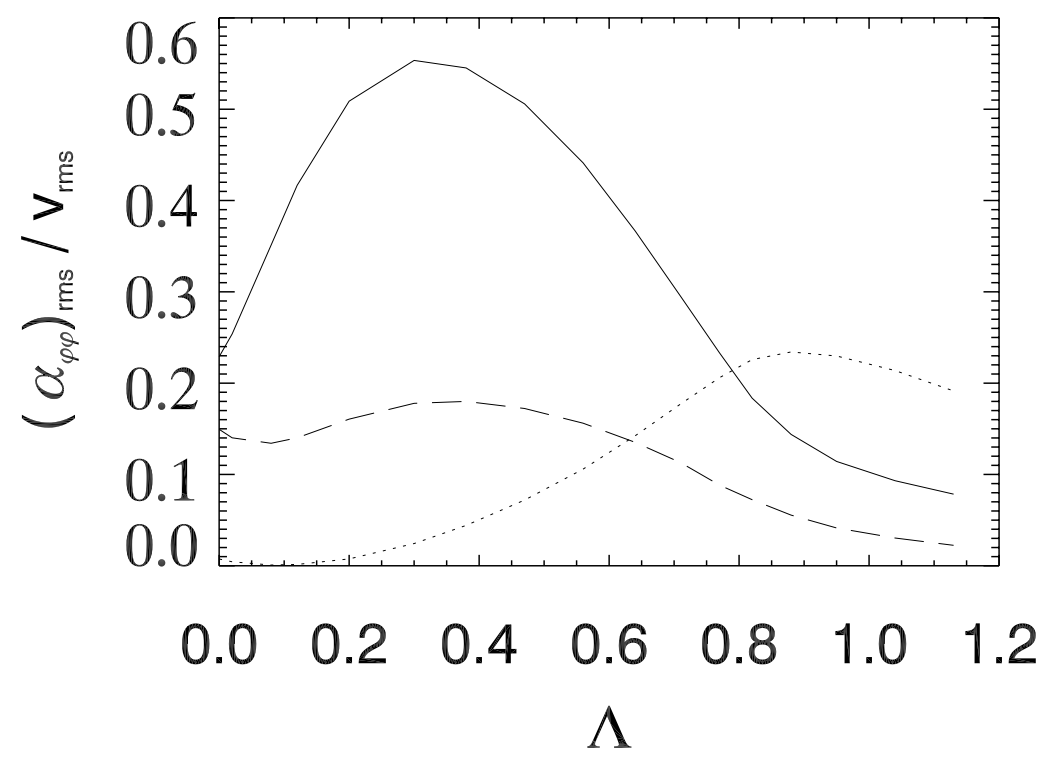

Figure 5.9: Solid line: r.m.s. value of $\alpha_{\varphi \varphi}$ measured in scales of $V_{\mathrm{rms}}$ in dependence of the Elsasser number $\Lambda$, dashed line: non-axisymmetric kinetic energy density, dotted line : axisymmetric kinetic energy density; both energy densities are given in scales of $1 / 300 \varrho \nu^{2} / D^{2}$. Coincidently with the non-axisymmetric kinetic energy density, $\left(\alpha_{\varphi \varphi}\right)_{\mathrm{rms}} / V_{\mathrm{rms}}$ experiences an increase at low field strengths while it is effectively quenched for $\Lambda>0.8$.

conflicts the Proudman-Taylor theorem. However, the presence of a magnetic field relaxes the geostrophic constraint and thus leads to a decrease of the critical Rayleigh number at low field strengths (Fearn 1998). For higher values of $\Lambda,\left(\alpha_{\varphi \varphi}\right)_{\text {rms }}$ is indeed quenched and drops rapidly until the decrease flattens again at $\Lambda>0.8$. At the same time, the axisymmetric kinetic energy density increases and a strong azimuthal flow develops, while convection is more and more suppressed. During the phase of rapid decay, the r.m.s value of $\alpha_{\varphi \varphi}$ in units of $V_{\text {rms }}$ scales relative to its maximum value $\alpha_{\max }=0.55$ at $\hat{B}_{\max }=2.6$ according to

$$
\left(\alpha_{\varphi \varphi}\right)_{\mathrm{rms}}=\frac{\alpha_{\max }}{1+k\left(\hat{B}-\hat{B}_{\max }\right)^{p}}
$$

in which $\hat{B}$ means the r.m.s. value of the mean field normalised with the equipartition value, and the parameters $k$ and $p$ are found to be $k \approx 1.4, p \approx 2.8$. 


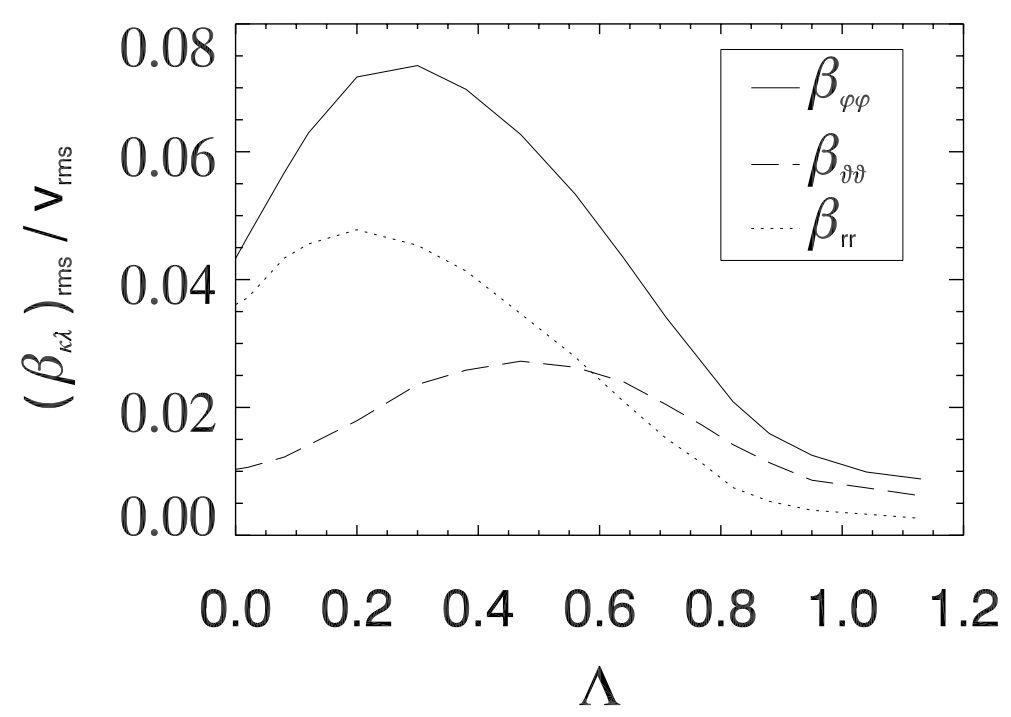

Figure 5.10: Diagonal components of the $\beta$-tensor normalised by $V_{\text {rms }}$, in units of the shell width $\mathrm{D}$, against the Elsasser number $\Lambda$. The quenching curves resemble those of the $\alpha$-quenching, compare Figure 5.9.

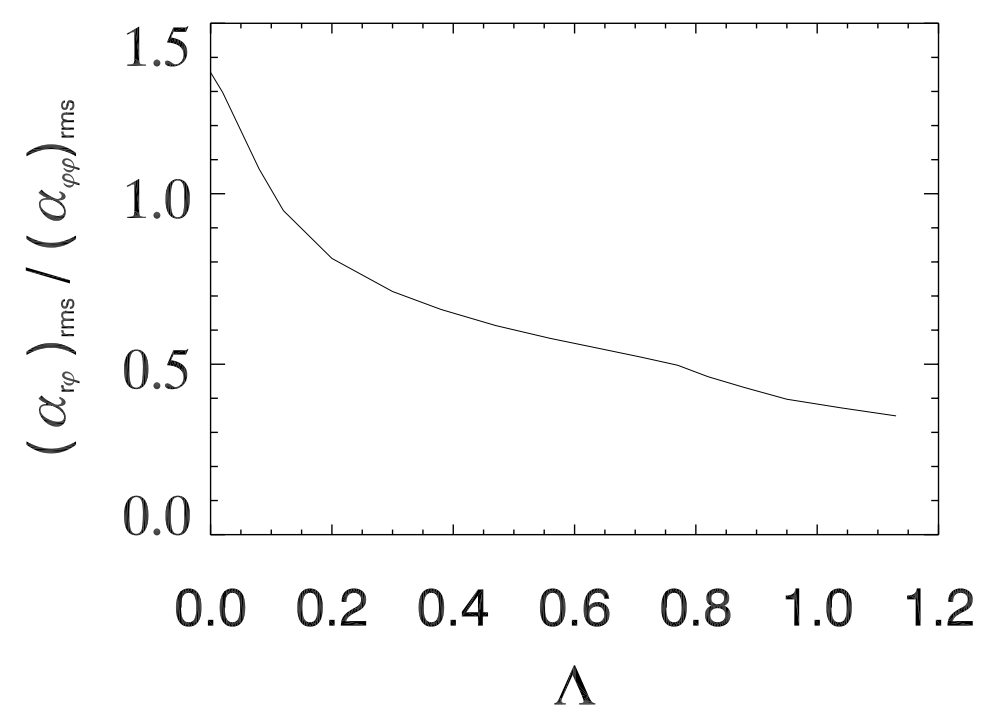

Figure 5.11: Mean-field coefficients are quenched in different ways. Displayed is the ratio of $\left(\alpha_{r \varphi}\right)_{\mathrm{rms}}$ to $\left(\alpha_{\varphi \varphi}\right)_{\mathrm{rms}}$ in dependence of $\Lambda$ 

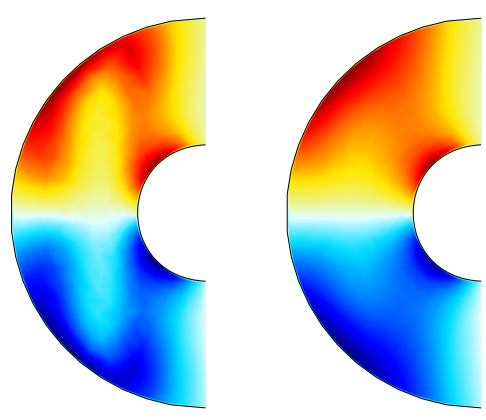

Figure 5.12: Mean azimuthal field for $\Lambda_{0}=1$ (left) and $\Lambda_{0}=2$ (right). While magnetic flux is expelled from the dynamo region in the first example, the imposed field remains rather undisturbed in the right chart.

The radial magnetic field for $\Lambda=1.2$ is displayed in Figure 5.13. Although the corresponding $\alpha$-components are already markedly quenched, the resulting maximum field strength has only decreased by less than $20 \%$ or rather less than $40 \%$, if only the axisymmetric part of the poloidal field is considered. This comparatively large field strength is interpreted partly as a consequence of the $\beta$-quenching, which likewise reduces the turbulent diffusivity. The r.m.s. values of the diagonal components of the $\beta$-tensor in dependence of $\Lambda$ are shown in Figure 5.10.

Apart from their similar field strengths, the simulation runs for $\Lambda_{0}=1$ and $\Lambda_{0}=2$ differ in their power spectra of the magnetic energy density as can already be inferred by a comparison between Figures 5.3 and 5.13. In the latter example, the power of the axial dipole mode has fallen below the non-axisymmetric contributions with harmonic degree $l=7$ and $l=8$, which lead to pronounced flux patches at mid and low latitudes. Because non-axisymmetric portions of the magnetic field are not directly linked to the mean-field coefficients presented here, there is no straightforward explanation for the concentration of the radial field at low latitudes in Figure 5.13. However, there is an axisymmetric parallel: While the imposed azimuthal field is diffused and swept out of the dynamo region in the example with $\Lambda_{0}=1$, it remains nearly undisturbed for $\Lambda_{0}=2$. In order to explain this change of the toroidal field configuration, it is of interest to consider the variation of $\gamma_{M T}$ with increasing $\Lambda$. Figure 5.11 displays the ratio $\left(\alpha_{r \varphi}\right)_{\mathrm{rms}} /\left(\alpha_{\varphi \varphi}\right)_{\mathrm{rms}}$ in dependence of $\Lambda$ demonstrating that both components are quenched differently. While $\alpha_{r \varphi}$ dominates roughly by a factor $3 / 2$ at $\Lambda=0$, this relation drops to less than $1 / 2$ for higher values of $\Lambda$. Let's recall that $\alpha_{r \varphi}$ in $\gamma_{M T}$ is related to the conflicting combination of columnar convection and spherical boundaries, which compels a meridional flow convergent to the equatorial plane in upflows near the outer boundary. This effect is moderated for growing $\Lambda$ because convection becomes less columnar, while the initially stimulated convection leads to an increase of $\alpha_{\varphi \varphi}$ at the same time. As a result, $\left(\alpha_{r \varphi}\right)_{\mathrm{rms}} /\left(\alpha_{\varphi \varphi}\right)_{\mathrm{rms}}$ decreases with the steepest descent in the range of $\Lambda<0.2$. Consequently, this quenching of $\alpha_{r \varphi}$ above average allows for the magnetic flux to remain in the dynamo region at mid latitudes and radii. It will be proven in Chapter 7 that advection due to $\gamma_{\mathrm{MT}}$ is indeed responsible for the specific pattern of the diffused azimuthal field visible in Figure 5.12. 


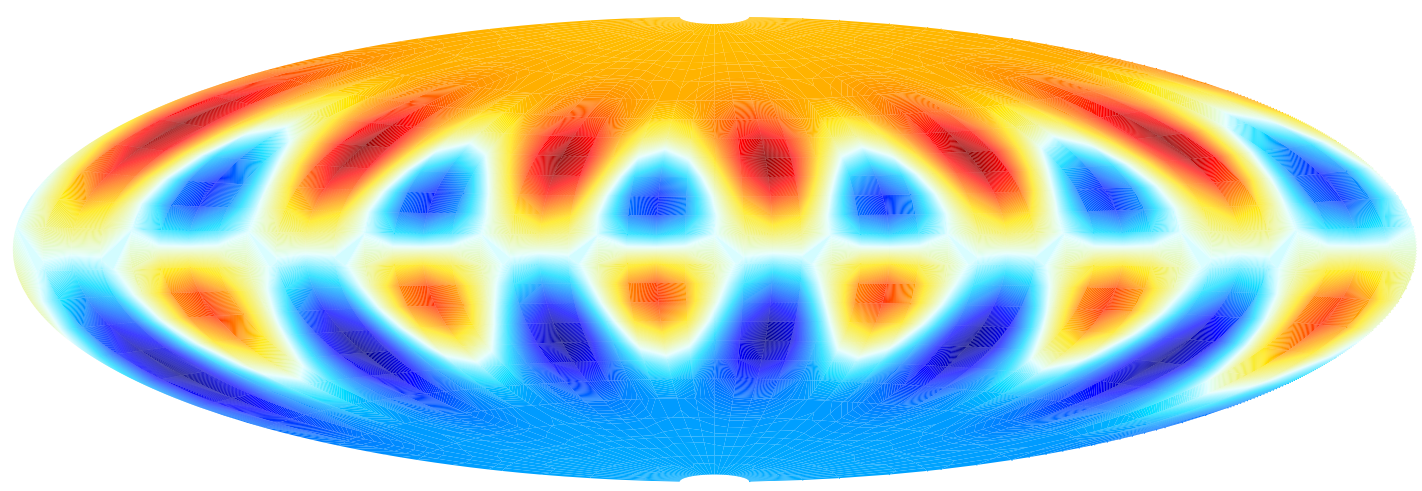

Figure 5.13: The radial magnetic field in the magnetoconvection example at $r=0.59 r_{o}$ for $\Lambda_{0}=2.0$. Its maximum field strength is given by $B_{r}=0.53(\varrho \mu \eta \Omega)^{1 / 2}$ and thus only slightly decreased compared to the simulation run with $\Lambda_{0}=1$, see also Figure 5.3. However, the spectrum of the magnetic energy density changed markedly: The axial dipole field has decreased relatively to the radial flux patches at mid and low latitudes.

\subsection{A simple quasi-stationary dynamo}

\subsubsection{Characteristics of the dynamo and supplements to approach (I)}

A very simple dynamo is examined in a further example. In Figure 5.14, the radial velocity field at $r=0.61 r_{o}$ is shown, revealing the columnar structure of the convection pattern. The governing parameters have been chosen to be $E=10^{-3}, R a=100$ (= $\left.1.79 R a_{c}\right), \operatorname{Pr}=1$, and $P m=5$. The intensity of the fluid motion can be characterised by $R m \approx 40$, and the magnetic energy density exceeds the kinetic one by a factor of 20. Again, except for a $\varphi$-drift of the convection columns, the velocity field is stationary. This example has been used before as a numerical dynamo benchmark and is taken from Christensen et al. (2001). In the following, it is denoted as benchmark example.

Note that in a kinematic treatment, the example under consideration turns into a slightly subcritical dynamo, and the magnetic energy density decreases exponentially. It is the $\varphi$-drift of the non-axisymmetric magnetic field $\boldsymbol{b}$ due to a constant mean azimuthal flow in the dynamo region, which leads to an altered, reduced electromotive force, if the velocity field is held constant. But, this deficit can be compensated by a transformation to the corotating frame of reference. Again, inhomogeneous boundary conditions for the toroidal velocity potential according to (5.4) have been adopted with a drift frequency $\omega=-3.17 \nu / D^{2}$. For this transformed velocity field, the benchmark example indeed proves to be a stationary dynamo, even in a kinematic treatment.

Alternatively, the benchmark example has been treated as a time dependent dynamo, making use of the generalisation of approach (I) to time dependent velocity fields. The resulting mean-field coefficients equal those obtained for a stationary velocity field in the corotating frame of reference. Various tests suggest that the time-dependent solutions of (4.3) become in fact independent of their initial values as soon as a statistical equilibrium 


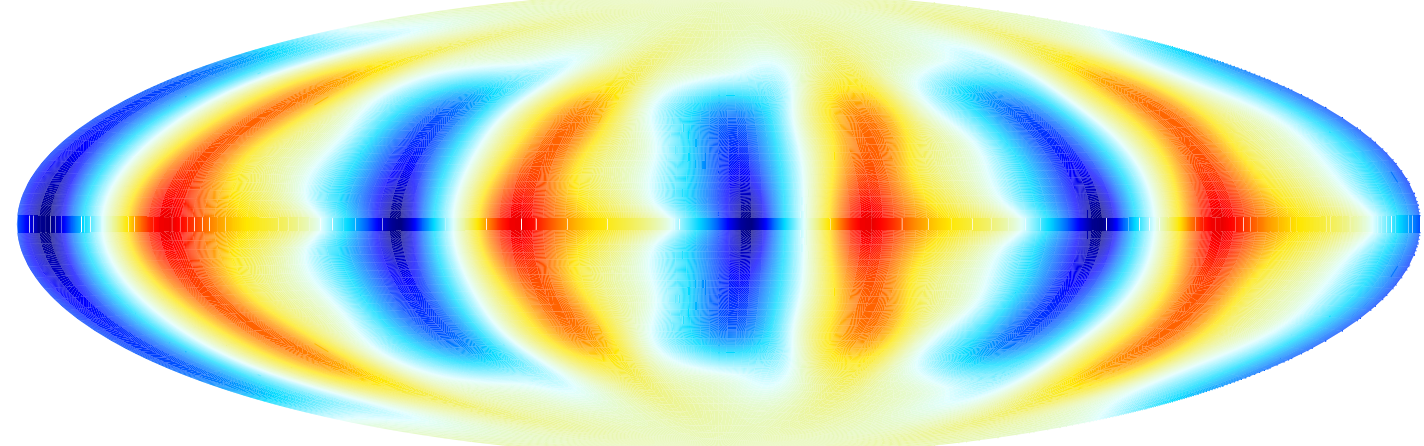

Figure 5.14: The radial velocity in the example of a simple (quasi-)stationary dynamo (benchmark example) at $r=0.61 r_{o}$. The convection pattern exhibits a natural 4-fold longitudinal symmetry and the absolute maximum is given by $V_{r}=16.99 \nu / D$.

is reached.

\subsubsection{Mean-field coefficients}

Six independent components of the symmetric $\alpha$-tensor and the 3 components of the $\gamma$ vector are shown in Figure 5.15. Among the $\alpha$-components, $\alpha_{\varphi \varphi}$ dominates, indicating a very efficient generation of poloidal out of toroidal magnetic field. Somewhat lower in amplitude are the components $\alpha_{r r}$ and $\alpha_{\vartheta \vartheta}$ which complete the $\alpha^{2}$-dynamo mechanism. This imbalance in the amplitudes of the diagonal components is reflected in the larger strength of the axisymmetric poloidal field compared to the axisymmetric toroidal field. Since the influence of differential rotation on the generation of toroidal field is negligible, as will be shown in Chapter 7, the benchmark example can be classified as an $\alpha^{2}$-dynamo.

Among the non-diagonal components, as before in the example of magnetoconvection, $a_{r \varphi}$ and $a_{\vartheta \varphi}$ are the most dominant coefficients. They govern the $\gamma$-vector which acts to expel flux from the dynamo region where convection takes place. Further meanfield coefficients, the $\beta$ - and $\kappa$-tensors as well as the $\delta$-vector, are given in Appendix F.

\subsubsection{Beyond $\tilde{a}$ and $\tilde{b}$ : the expansion of $\mathcal{E}$ including derivatives of $\bar{B}$ up to the second order}

The expansion of $\mathcal{E}$ has been extended considering also derivatives of second order by means of the 9 additional test fields (4.5-4.13). I abstain here from displaying the 27 mean-field coefficients related to the second order derivatives $\partial^{2} \bar{B}_{\lambda} / \partial r^{2}, \partial^{2} \bar{B}_{\lambda} / \partial r \partial \vartheta$ and $\partial^{2} \bar{B}_{\lambda} / \partial \vartheta^{2}$. However, two points should be reported:

- The electromotive forces related to the second order test fields (4.5-4.13) show only minor deviations from parametrisations including derivatives up to the first order 

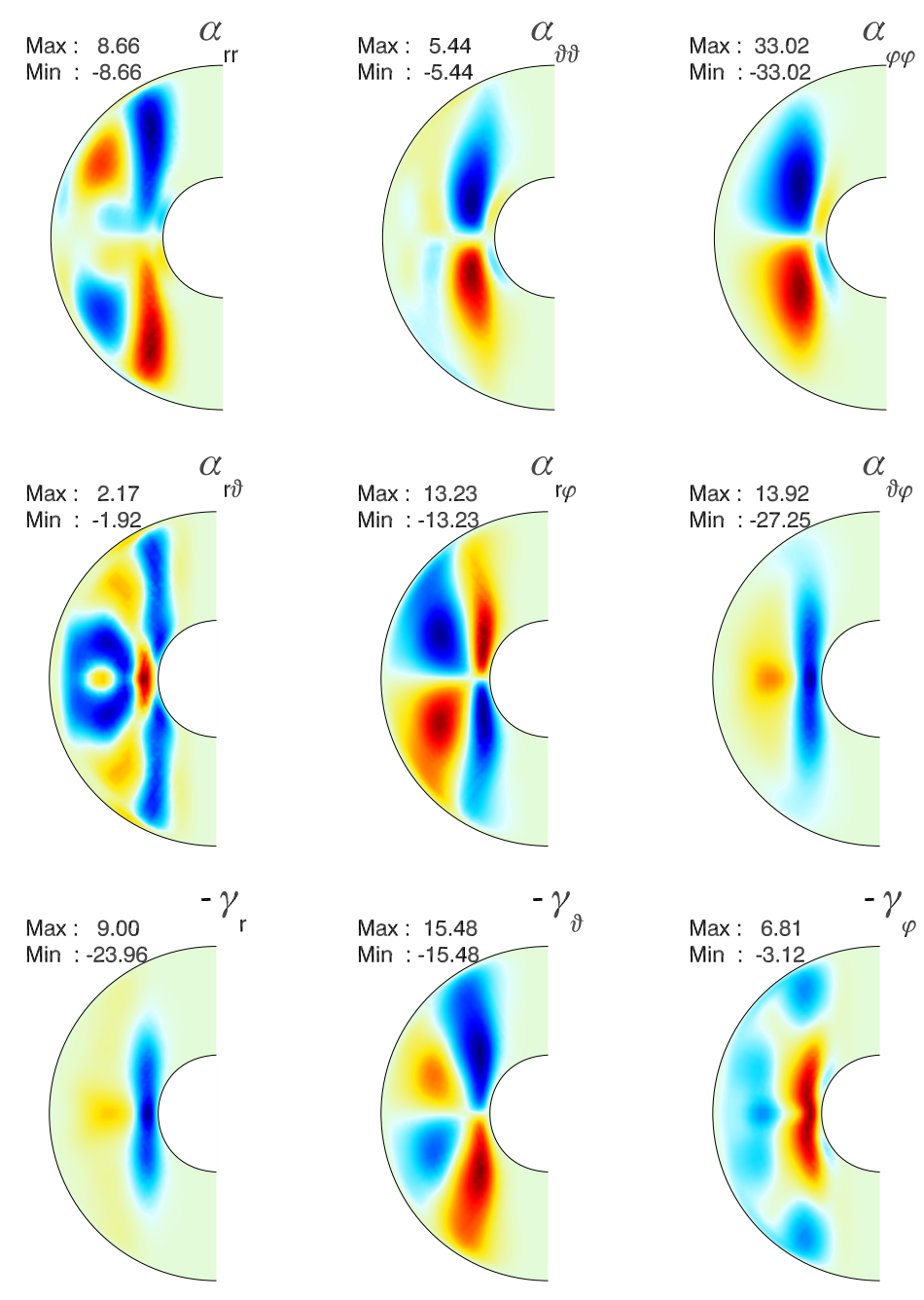

Figure 5.15: Six independent components of the symmetric $\alpha$-tensor and the $\gamma$-vector in the benchmark example in units of $\nu / D$.

only,

$$
\mathcal{E}_{\kappa}^{(1)}=\tilde{a}_{\kappa \lambda}\left(\bar{B}_{T}\right)_{\lambda}+\tilde{b}_{\kappa \lambda r} \frac{\partial\left(\bar{B}_{T}\right)_{\lambda}}{\partial r}+\tilde{b}_{\kappa \lambda \vartheta} \frac{1}{r} \frac{\partial\left(\bar{B}_{T}\right)_{\lambda}}{\partial \vartheta}
$$

with prior determined coefficients $\tilde{a}_{\kappa \lambda}, \tilde{b}_{\kappa \lambda \mu}$ and second order test fields $\overline{\boldsymbol{B}}_{\boldsymbol{T}}$. Thus, the new coefficients $\tilde{c}_{\kappa \lambda r r}, \tilde{c}_{\kappa \lambda r \vartheta}$, and $\tilde{c}_{\kappa \lambda \vartheta \vartheta}$ are small in amplitude compared to $\tilde{a}_{\kappa \lambda}$ and $\tilde{b}_{\kappa \lambda \mu}$, that is, contributions to $\mathcal{E}$ from second order derivatives are negligible, provided that $\partial_{\mu} \partial_{\nu} \bar{B}_{\lambda} \sim O\left(\bar{B}_{\lambda}\right)$. Note that this decline in amplitude is consistent with the idea of a rapidly converging series expansion of $\mathcal{E}$.

- All components $\tilde{c}_{\kappa \lambda \mu \nu}$ possess well defined symmetries with respect to the equator in accordance with the symmetry considerations in Section 3.4. This is not evident at the first place but confirms again approach (I), since the test fields and thus the related electromotive forces do not possess such symmetries. 


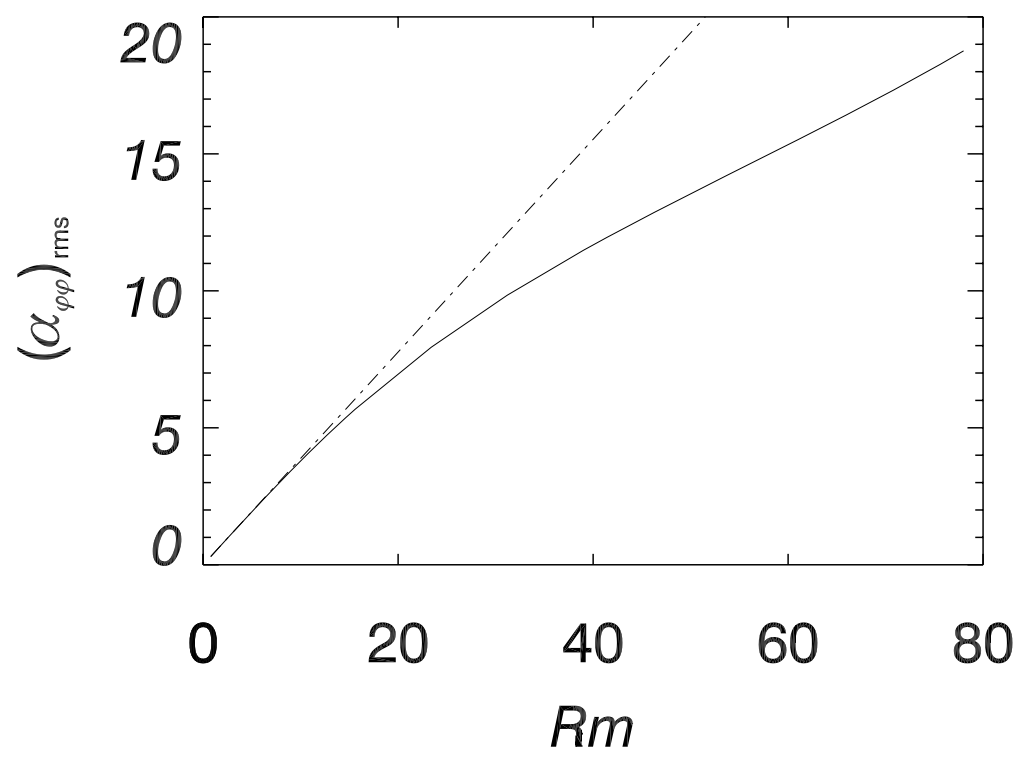

Figure 5.16: The quantity $\left(\alpha_{\varphi \varphi}\right)_{\mathrm{rms}}$ in the benchmark example in units of $\nu / D$, with $\alpha_{\varphi \varphi}$ determined by method (I) and (II) (solid and dashed line, respectively) in dependence of $R m$.

\subsubsection{Shortcomings due to SOCA}

Most mean-field models rely on mean-field coefficients derived in the second order correlation approximation although this might not be justified by the properties of the underlying flow. Therefore, it is of interest to point out in which way mean-field coefficients are spoiled by the application of SOCA for a stationary flow with $R m \gg 1$, for instance. For the examples considered here, mean-field coefficients determined by approach (I) and approach (II) show an almost perfect congruence of their profiles in a meridional plane. On the other hand, mean-field coefficients determined by means of SOCA exhibit typically overestimated amplitudes for $R m \gg 1$.

In Figure 5.16, $\left(\alpha_{\varphi \varphi}\right)_{\mathrm{rms}}$ is plotted against $R m$, which is again varied by varying $P m$. For small $R m$, both results merge and follow the linear dependence on $P m$ as prescribed by (D.9). But, for $R m \approx 10$, the slope of $\left(\alpha_{\varphi \varphi}\right)_{\mathrm{rms}}$ determined by approach (I) flattens. Thus, $\alpha_{\varphi \varphi}$ derived without applying SOCA leads to amplitudes which are about $30 \%$ smaller compared to the respective SOCA-calculations for $R m \approx 40$. The resultant consequences for the dynamo action in a mean-field model will be studied in Chapter 7 .

Before completing this Section, two warnings are given concerning the apparent linear dependence of $\alpha_{\varphi \varphi}$ on $R m$ in the SOCA-limit:

- All mean field-coefficients determined by approach (II) scale with $v^{2} / \eta$ in which $v$ is meant to be a typical velocity scale, as can be inferred from (D.2-D.9). A rescaling of $\boldsymbol{V}$ instead of the diffusivity will therefore result in a quadratic dependence of 


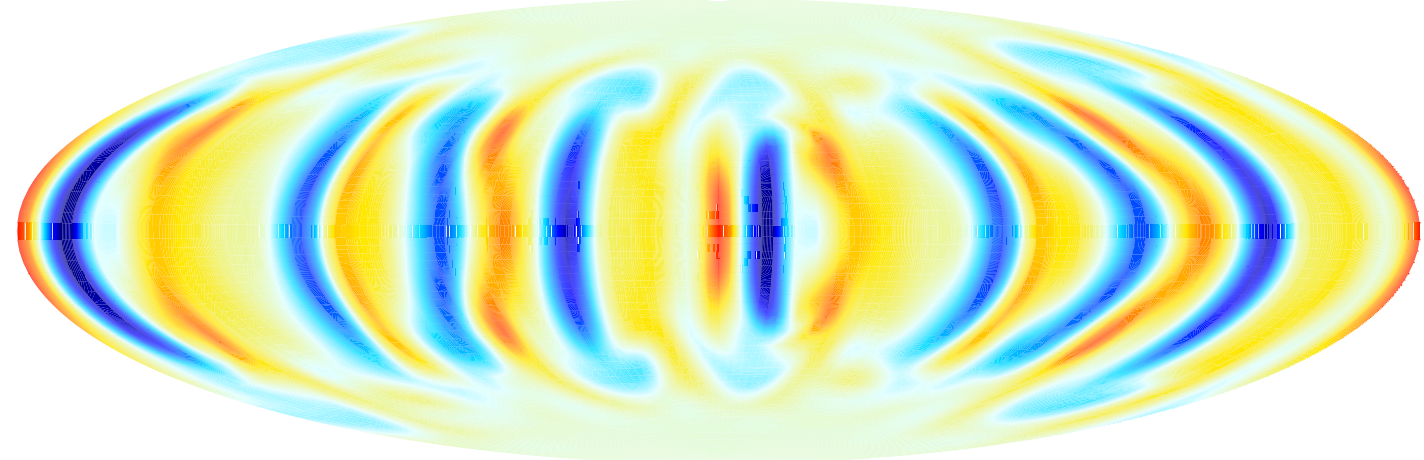

Figure 5.17: Snapshot of the radial velocity field in the example of a strongly columnar but highly time-dependent dynamo at $r=0.58 r_{o}$. The absolute maximum is given by $V_{r}=99.33 \nu / D$. The velocity field is symmetric about the equatorial plane although no symmetry conditions have been imposed.

$\left(\alpha_{\varphi \varphi}\right)_{\mathrm{rms}}$ on $R m$.

- The reciprocal linear dependence of (D.2-D.9) on the magnetic diffusivity is due to the presumed stationarity of $\boldsymbol{b}$. This is best illustrated by a comparison between (3.40) and (3.43). While $\alpha_{I}$ depends linearly on Pm in the low conductivity limit, there is no dependence on $\mathrm{Pm}$ at all in the high conductivity limit. Both results are limiting cases and do not reflect the general situation. However, a generalisation of approach (II) for time-dependent velocity fields is possible and will be discussed in Chapter 8.

\subsection{A highly time-dependent dynamo in the strongly colum- nar regime}

\subsubsection{Characteristics of the dynamo and mean-field coefficients}

The next example is again a strongly columnar dynamo resembling the benchmark example in many respects. The governing parameters are given by $E=10^{-4}, R a=334$ (= 4.8R $\left.a_{c}\right), \operatorname{Pr}=1$ and $\operatorname{Pm}=2$, see also Olson et al. (1999). The magnetic energy exceeds the kinetic energy roughly by a factor of three, and the magnetic field has a large axial dipole contribution. The magnetic Reynolds number has doubled compared to the benchmark dynamo and is about $R m \approx 88$. A snapshot of the radial velocity field is plotted in Figure 5.17. Although convection is highly time-dependent, the velocity field is still symmetric about the equatorial plane. This equally implies well defined symmetries for the mean-field coefficients for any time. The time-averaged $\alpha$-tensor and $\gamma$-vector presented in Figure 5.19 are very similar to the coefficients for the benchmark example shown in Figure 5.15, thus suggesting similar dynamo processes. Differences occur in the 


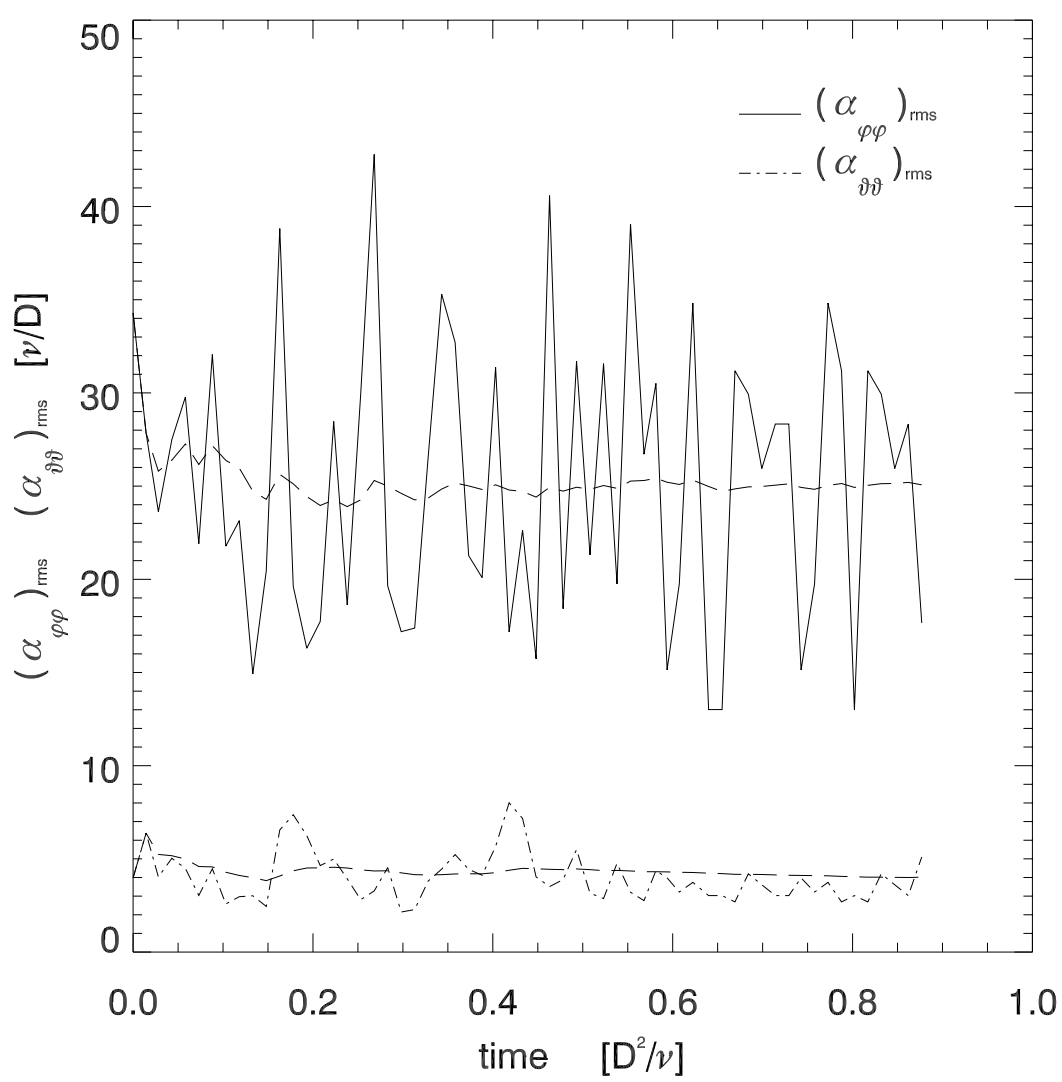

Figure 5.18: A time series of the spatially averaged mean-field coefficients $\alpha_{\varphi \varphi}$ and $\alpha_{\vartheta \vartheta}$ together with the corresponding running averages (dashed lines). Here, the running average at time $t=t_{1}$ is taken over the time intervall $\left[0, t_{1}\right]$. The time series consists in a number of $60 \alpha$-tensors separated by time steps $\Delta t=1.5 \times 10^{-2}$. Smaller time steppings have been adopted as an experiment in order to rule out fluctuations on much smaller time scales.

$\alpha_{\vartheta \vartheta}$-component, which is markedly reduced in amplitude compared to $\alpha_{r r}$. Moreover, the convection columns and so the mean-field coefficients appear to be more elongated due to a smaller Ekman number. However, as in the benchmark example, the dynamo under consideration seems to operate again by an $\alpha^{2}$-mechanism with dominant $\alpha_{\varphi \varphi}$-component, in agreement with the interpretation given already by Olson et al. (1999). In addition, a strong $\gamma$-effect influences the magnetic field as has been discussed earlier.

\subsubsection{Time-variability of mean-field coefficients}

The mean-field coefficients vary typically on a time scale of $\tau=1.5 \times 10^{-2} D^{2} / \nu$, which is of the same order as the convective turnover time estimated roughly through $D /\left|V_{r}\right|^{\max }$. In Figure 5.18, a time series of the spatially averaged components $\alpha_{\vartheta \vartheta}$ and $\alpha_{\varphi \varphi}$ is presented. The running time averages converge rapidly and become nearly constant within 

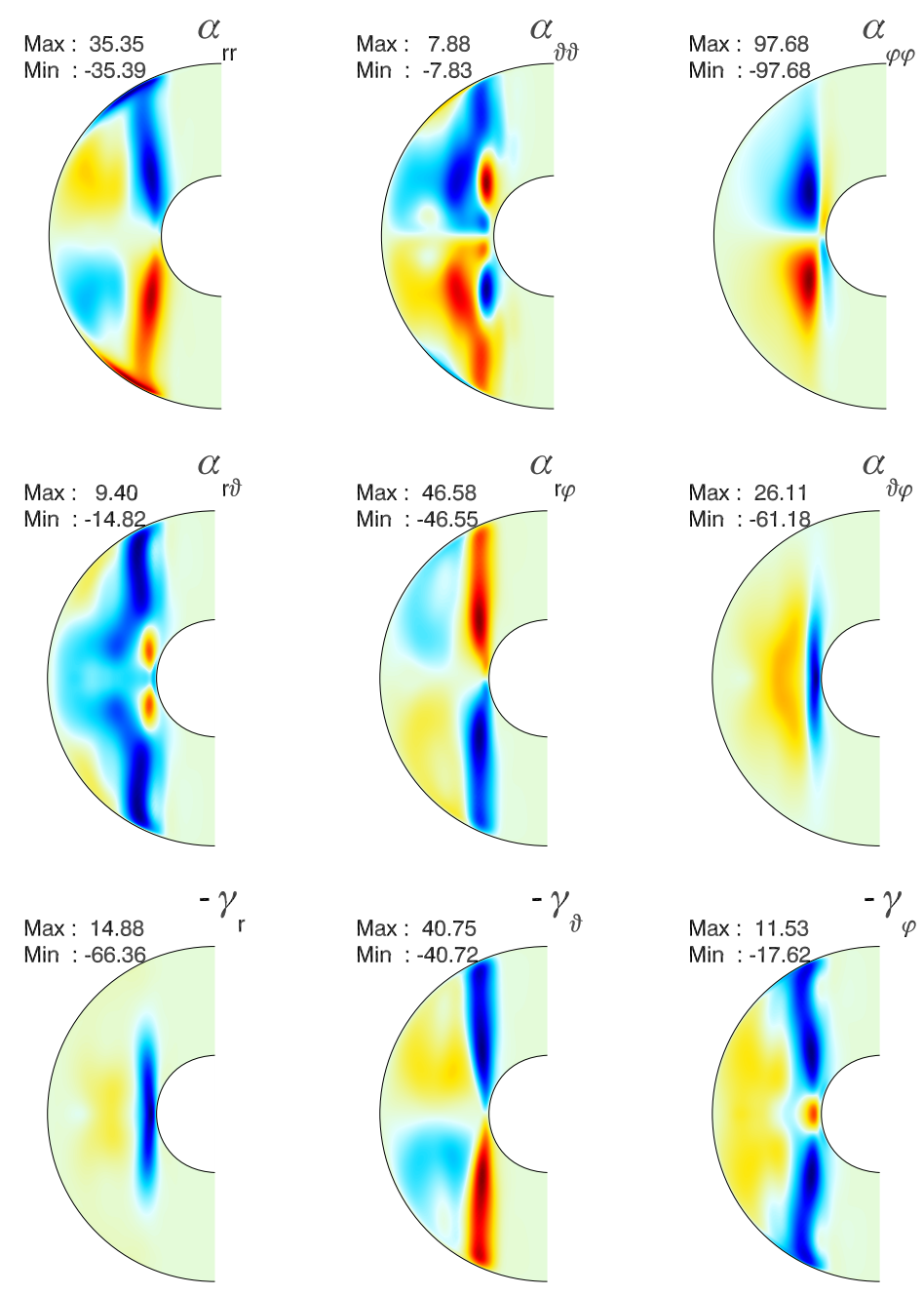

Figure 5.19: Six independent components of the symmetric $\alpha$-tensor and the $\gamma$-vector in the example of a highly time-dependent dynamo in units of $\nu / D$. The mean-field coefficients displayed here have been averaged in time over a time period of $\tau=0.9 D^{2} / \nu$.

a time period of $\tau=0.9 D^{2} / \nu$. Nevertheless, there are fluctuations around the running average with amplitudes exceeding it by up to $80 \%$. A more detailed view is provided by Figure 5.20 displaying the corresponding local standard deviations for the $\alpha$-tensor and $\gamma$-vector in the meridional plane. It reveals clear differences in the relative strength of the fluctuations in time among the $\alpha$ - and $\gamma$-components. While the dominating coefficients $\alpha_{\varphi \varphi}, \alpha_{\vartheta \varphi}$ and $\gamma_{r}$ fluctuate moderately with standard deviations of less then $50 \%$, the more insignificant coefficients $\alpha_{\vartheta \vartheta}, \alpha_{r \vartheta}$ and $\gamma_{\varphi}$ show standard deviations being at least twice as high.

Since all fluctuations in time obey the symmetry rules given in Table 3.1, the standard deviations exhibit strict equatorial symmetries as visible in Figure 5.20. Therefore, variations in the northern and southern hemisphere can not be taken as independent, and the contour plots in Figure 5.20 indicate also typical length scales on which fluctuations occur. For most components considered here, these are comparable in size with the di- 

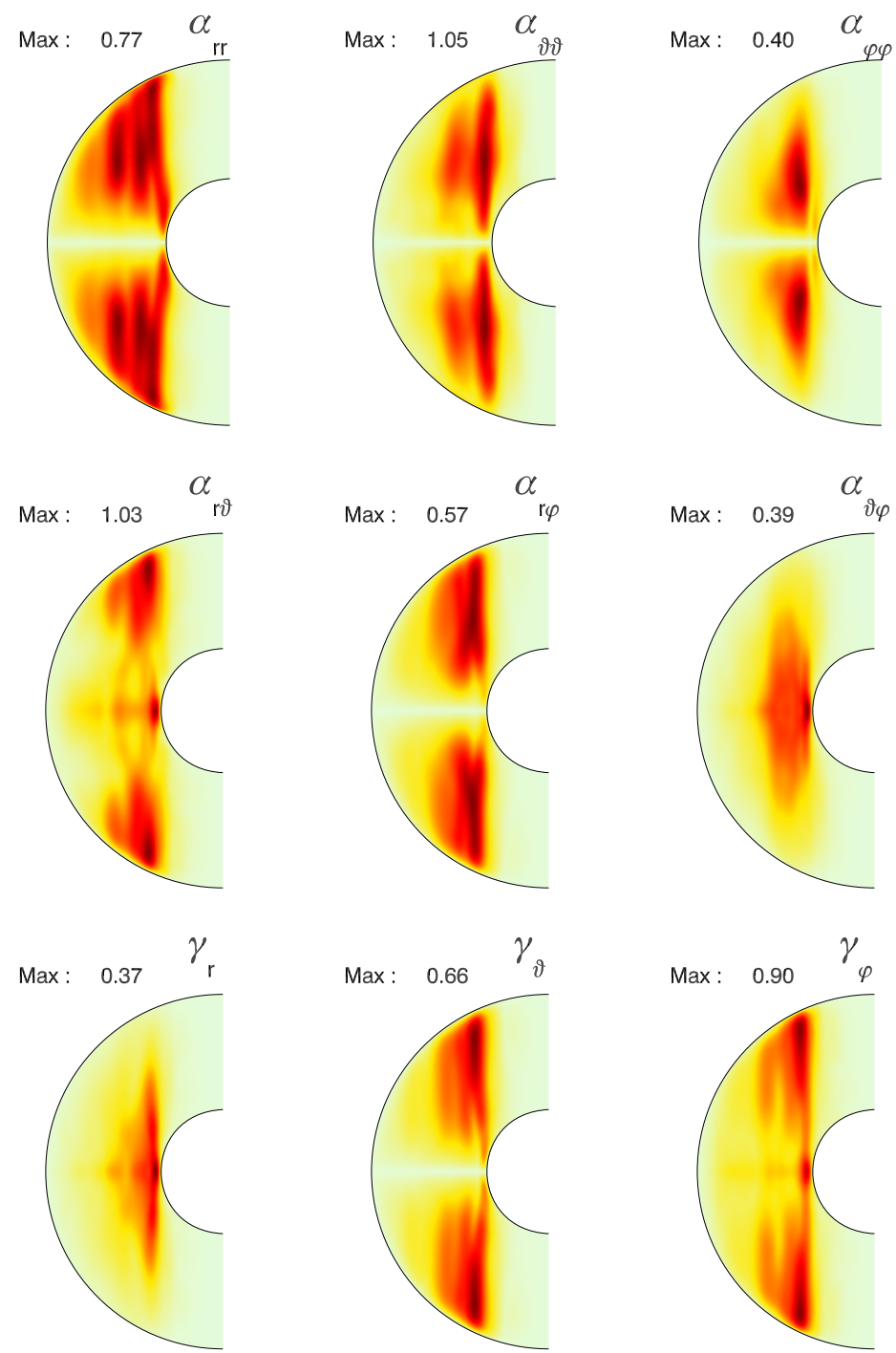

Figure 5.20: Standard deviations for fluctuations of $\alpha$ - and $\gamma$-components in time. The maximum standard deviations written next to each contour plot are normalised by the absolute maximum of each component.

ameter of the shell, that is, fluctuating mean-field coefficients in time vary on the same spatial scales as time-averaged coefficients. 


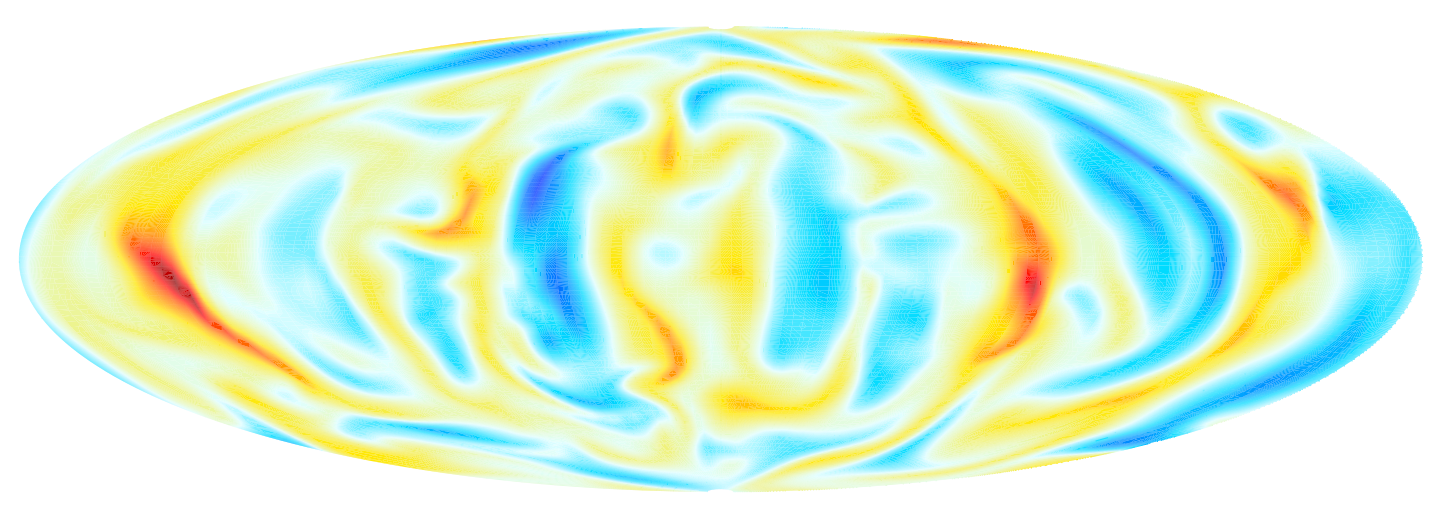

Figure 5.21: Snapshot of the radial velocity field at $r=0.55 r_{o}$ in the example of a more chaotic dynamo, which is less columnar and not symmetric about the equatorial plane. The absolute maximum is given by $V_{r}=1224 \nu / D$.

\subsection{A highly time-dependent dynamo in the fully devel- oped regime}

\subsubsection{Characteristics of the dynamo, adaption of approach (I), and resulting mean-field coefficients}

The fourth example considered here is again a very time-dependent dynamo which is no longer strongly columnar (see Figure 5.21). It is among the dynamos with reversing dipole field presented by Kutzner and Christensen (2002). The governing parameters read $E=3 \times 10^{-4}, R a=990\left(=16 R a_{c}\right), P r=1 / 3$ and $P m=1$. In this example, convection takes place also inside the inner core tangent cylinder, and the velocity field has lost its equatorial symmetry. Olson et al. (1999) refer to this regime as the fully developed regime. The magnetic Reynolds number, characterising the vigour of the fluid motion, is given by $R m=350$ and thus much higher than in the previous examples. But, this dynamo is much less effective: The averaged kinetic energy density exceeds the averaged magnetic energy density roughly by a factor of 50, and typically less than $10 \%$ of the magnetic energy is contributed by the axisymmetric field. Therefore, it may seem doubtful whether mean-field coefficients based on an axisymmetric mean reveal anything significant about the dynamo processes taking place. On the other hand, the dynamo changes its character abruptly if the axisymmetric portion of the magnetic field is permanently eliminated, see Figure 5.22. In this experiment, the magnetic energy density decays within a time period of $\tau=0.4 D^{2} / \nu$ by approximately one order of magnitude. But, the dynamo does not die out completely, and there is a small mean electromotive force even with zero mean field. In the expansion of $\mathcal{E}$, this has to be considered by an extra term $\mathcal{E}_{0}$ which is independent of $\overline{\boldsymbol{B}}$, and (3.22) has to be replaced by

$$
\mathcal{E}-\mathcal{E}_{0}=a \bar{B}+b \nabla \bar{B}+\cdots
$$




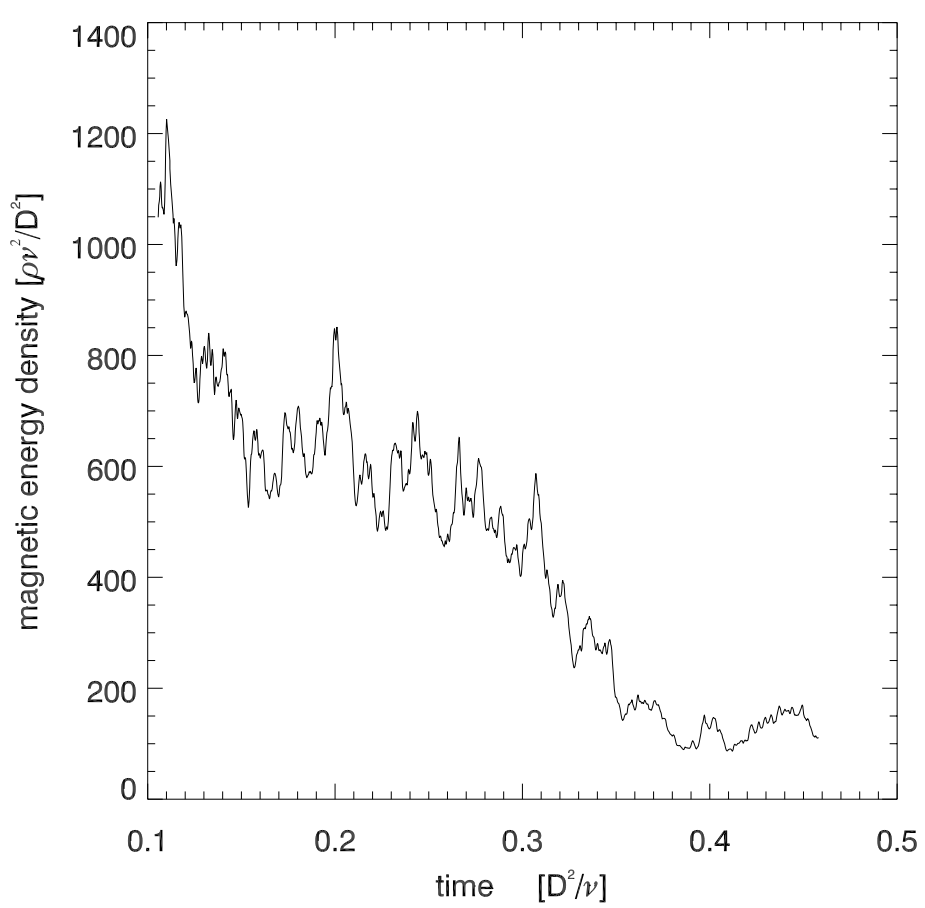

Figure 5.22: Decay of the magnetic energy density based on the non-axisymmetric field only. The particular induced axisymmetric portion of the field has been set to zero in each time step.

Consequently, the trivial test-field configuration $\overline{\boldsymbol{B}}_{T} \equiv 0$ must be added to the set of test fields given in Table 4.1 in order to determine mean-field coefficients by means of approach (I). Resulting components $\tilde{a}_{\kappa \lambda}$ obtained by an subsequent time-averaging are shown in Figure 5.23. Different from previous examples, the velocity field does not vanish close to the polar axis, which causes problems if $\nabla \times\left(\boldsymbol{v} \times \overline{\boldsymbol{B}}_{\boldsymbol{T}}\right)$ in equation (4.1) approaches a singularity there. Hence, regions close to the polar axis have been omitted, and the following discussion is based on the assumption that the induced electromotive forces inside and outside the inner core tangent cylinder are not strongly correlated.

The qualitative consistency of the time-averaged mean-field coefficients $\tilde{a}_{\kappa \lambda}$ in Figure 5.23 with previous examples strongly supports this assumption. Although mean-field coefficients given at any point in time vary on much smaller spatial scales and do not exhibit equatorial symmetries, see for instance Figure 5.28, the time averages in Figure 5.23 show characteristics which have been seen already before in the three previous examples: There are rather antisymmtric diagonal components which are positive in the northern and negative in the southern hemisphere, and the non-diagonal components $\tilde{a}_{r \varphi}$ and $\tilde{a}_{\vartheta \varphi}$ contribute to a $\gamma$-effect as discussed in Section 5.1.2. But, since the coefficients $\tilde{b}_{\kappa \lambda \mu}$ could not be equally well resolved, the corresponding covariant tensor components look partly less consistent, e.g. $\alpha_{\vartheta \vartheta}$ in Figure 5.25. For the time period of $\tau=0.25 D^{2} / \nu$ considered here, there is no clear dominance of any time-averaged coefficient in difference from previous examples. The high maximum amplitudes of $\tilde{a}_{r r}$ are only due to spatially very confined 

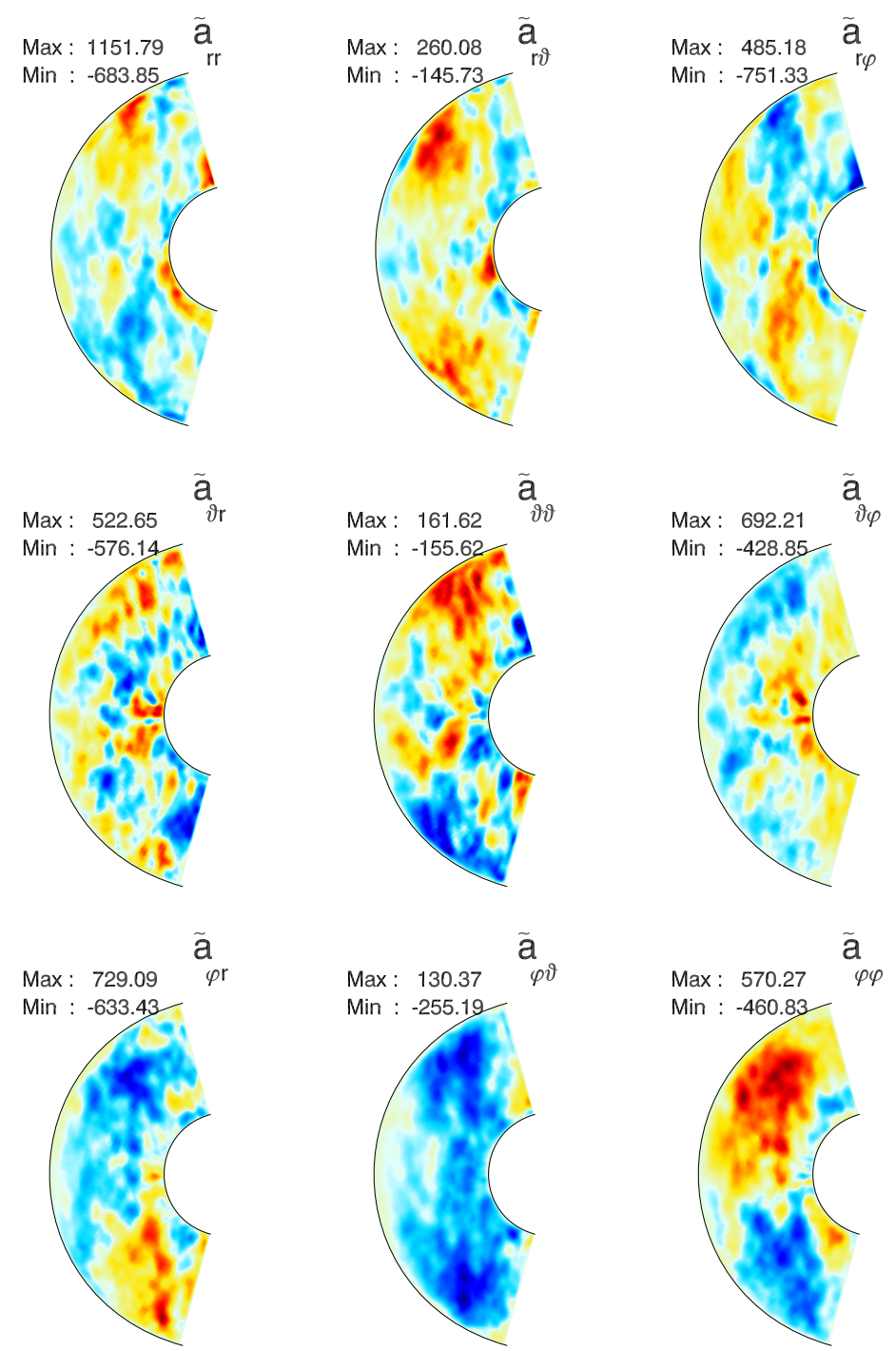

Figure 5.23: Time-averaged mean-field coefficients in the example of a highly time dependent dynamo in the fully developed regime. The time averaging has been carried out over a time period of $\tau=0.25 D^{2} / \nu$ and is based on 300 single data sets of mean-field coefficients varying in time. The maximum and minimum amplitudes are given in units of $\nu / D$. 

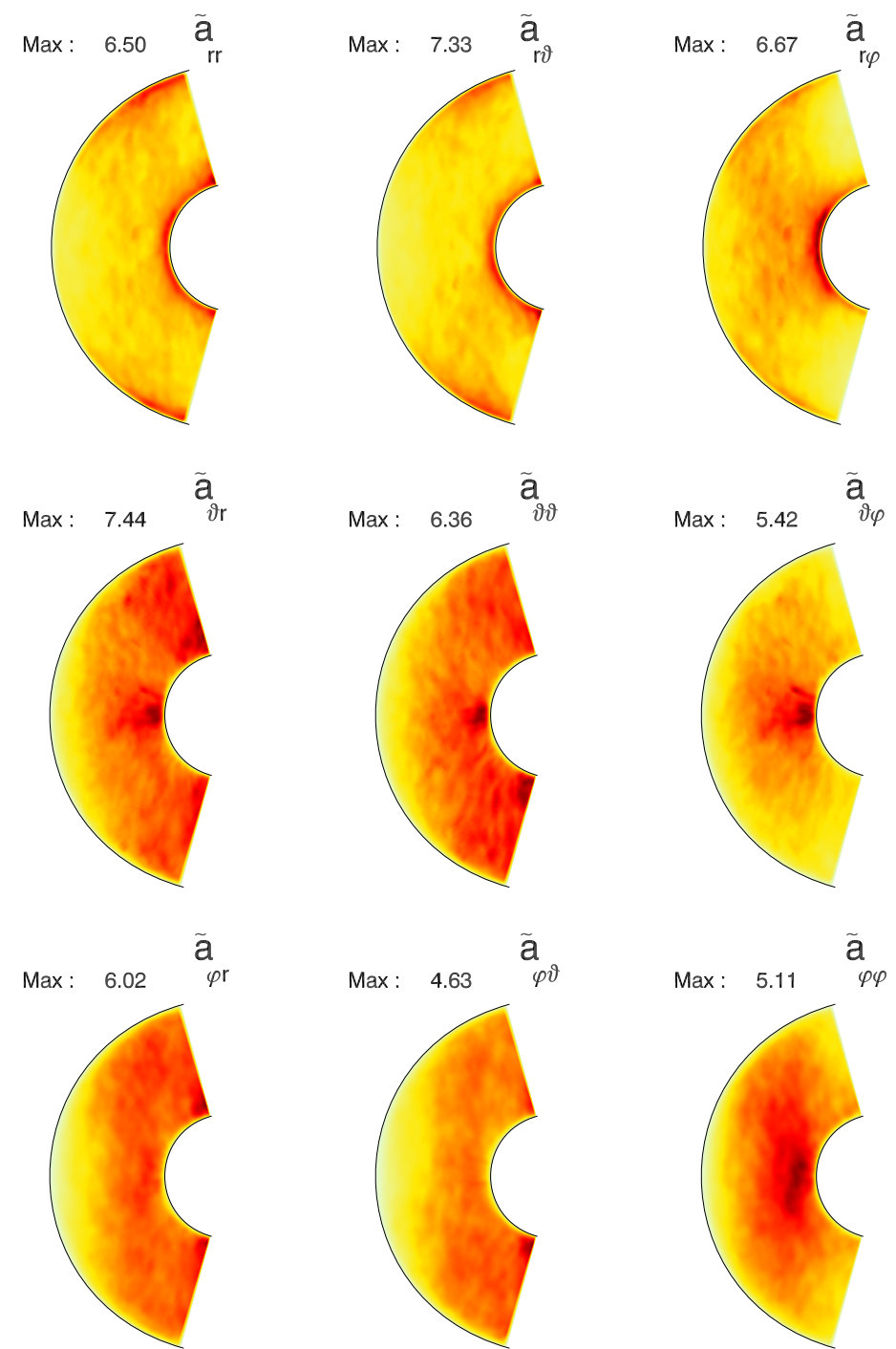

Figure 5.24: Standard deviations for the fluctuations of the components $\tilde{a}_{\kappa \lambda}$ around their time averages. The maximum standard deviations written next to each plot are normalised with the absolute maximum of each component. 

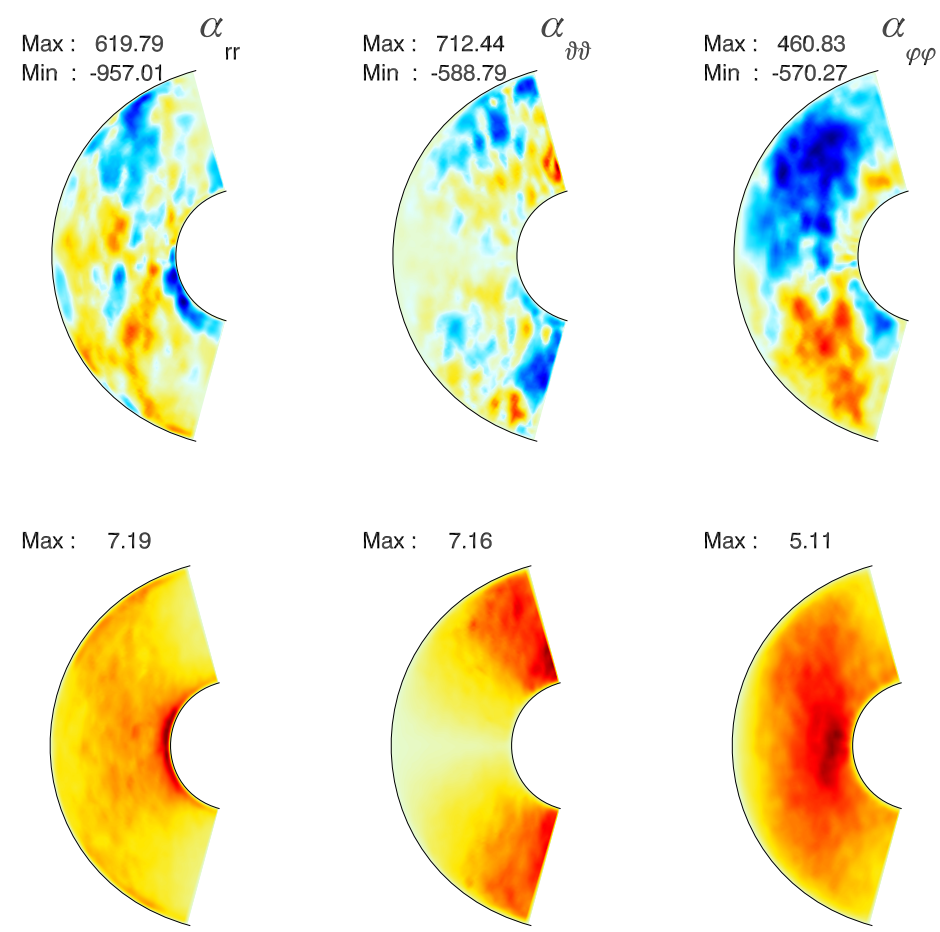

Figure 5.25: Time-averaged diagonal components of the $\alpha$-tensor together with their standard deviations (second row). Whereas $\alpha_{r r}$ and $\alpha_{\varphi \varphi}$ are antisymmetric about the equator and predominantly negative in the northern and positive in the southern hemisphere, as expected, $\alpha_{\vartheta \vartheta}$ is dominated by contributions from $\tilde{b}_{\vartheta r \vartheta}$ and requires further averaging. The maxima of the $\alpha$-components are given in units of $\nu / D$, and the maximum standard deviations are normalised by the absolute maximum of each component.

maxima, otherwise the component is rather equilibrated to $\tilde{a}_{\varphi \varphi}$. In contrast, $\tilde{a}_{\vartheta \vartheta}$ seems to be of less importance.

\subsubsection{Time variability of mean-field coefficients and reversals}

Figure 5.26 displays variations of $\left(\alpha_{\varphi \varphi}\right)_{\text {rms }}$ in time together with a running time average. Again, the running average at time $t=t_{1}$ is taken over a time intervall $\left[0, t_{1}\right]$. Figure 5.26 reveals chaotic fluctuations of $\left(\alpha_{\varphi \varphi}\right)_{\mathrm{rms}}$ on a time scale of the order $10^{-3} D^{2} / \nu$, which corresponds to a typical convective turnover time. In addition, $\left(\alpha_{\varphi \varphi}\right)_{\mathrm{rms}}$ changes on a larger time scale of the order $10^{-1} D^{2} / \nu$, and so, the running average could not be equilibrated for the rather small time period considered here. In contrast, a running average based on the absolute maxima of $\alpha_{\varphi \varphi}$ (dashed line) converges more rapidly, indicating that the time averaged $\alpha_{\varphi \varphi}$-component will probably not change markedly if the time interval is extended. This second variation on a larger time scale, which undergo all mean-field coefficients simultaneously, coincides with variations in the total and the axisymmetric magnetic energy density. A continuation of these calculations covering a larger time interval will be of future interest.

The huge standard deviations in Figure 5.24 and Figure 5.25 demonstrate the chaotic 


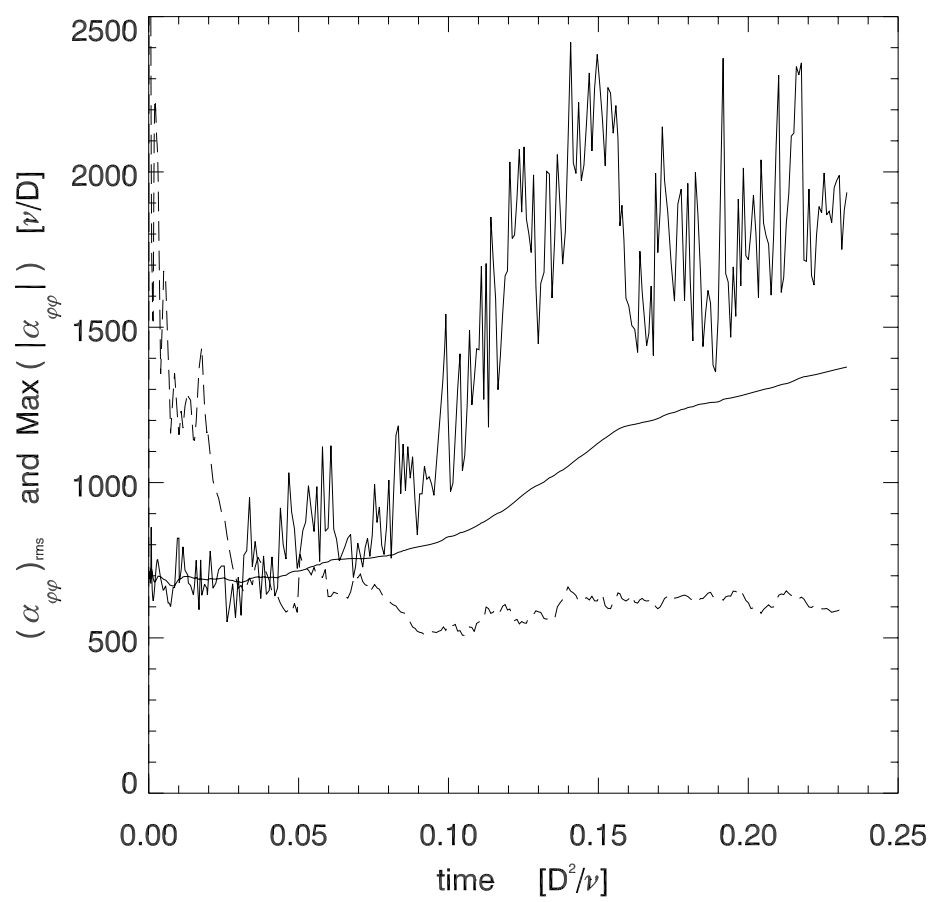

Figure 5.26: The spatially averaged component $\alpha_{\varphi \varphi}$ in dependence of time together with its running time average in the example of a dynamo in the fully developed regime. The dashed line represents a running average of the maximum norm applied to $\alpha_{\varphi \varphi}$, $\operatorname{Max}\left(\left|\alpha_{\varphi \varphi}\right|\right)$, which converges more rapidly.

time dependence of all mean-field coefficients. The standard deviation related to the $\alpha_{\varphi \varphi^{-}}$ component, for instance, exceeds five times its average in some regions of the meridional plane. Thus, the relative amplitudes of $\alpha_{\varphi \varphi}$-fluctuations in time are about one order of magnitude larger than the comparatively moderate fluctuations shown in Figure 5.20, referring to the example of a time dependent dynamo in the columnar regime.

A strongly fluctuating $\alpha$-coefficient is needed in a mean-field dynamo model presented by Hoyng et al. (2001) in order to reproduce certain characteristics of the variability of the axial dipole component of the geomagnetic field. According to their model, the amplitude of the fundamental dipole mode varies like the position of a stochastically driven particle in a bistable potential in which the minima represent reversed polarity states. Hoyng et al. (2001) succeed in predicting an observationally confirmed relation between secular variation and reversal rate as well as an amplitude distribution as given by the Sint- 800 record. However, the justification of the underlying assumption that the amplitudes of stochastic fluctuations in $\alpha$ exceed its average value by at least a factor of 2 was left as an unresolved issue. The calculations carried out here demonstrate that fluctuations of this strength indeed occur, thus supporting the model by Hoyng et al. (2001). 


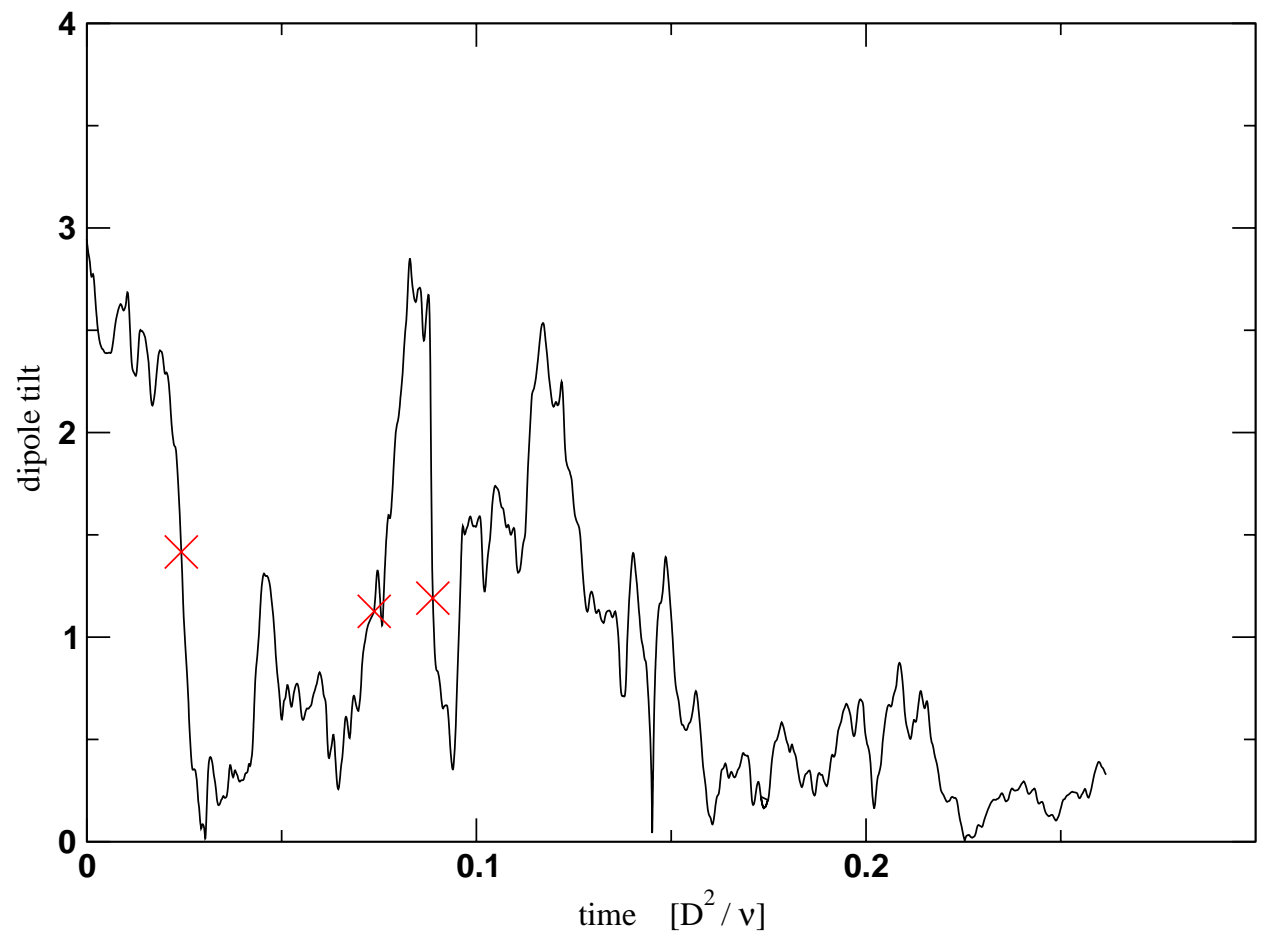

Figure 5.27: Tilt angle of the dipole-field axis in radian measure against time. The red crosses indicate points at which the snapshots of $\alpha_{\varphi \varphi}$ were taken shown in Figure 5.28 below.

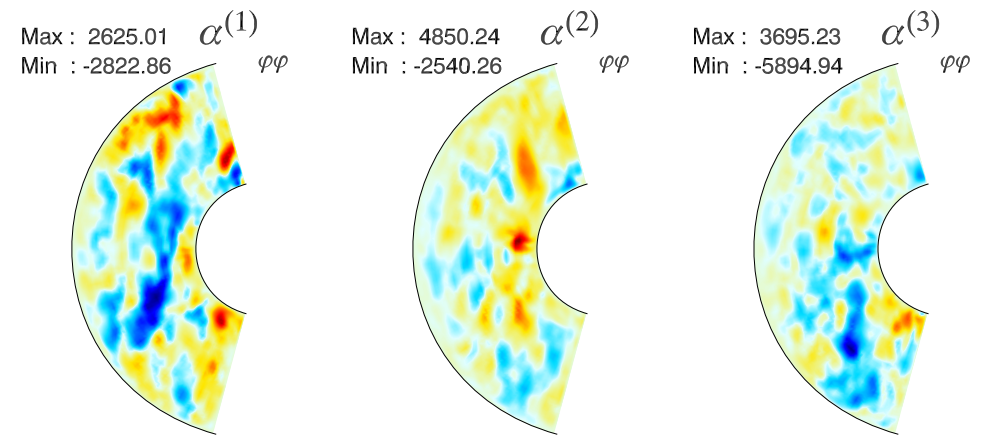

Figure 5.28: Three snapshots of $\alpha_{\varphi \varphi}$ for $t^{(1)}=0.02 D^{2} / \nu, t^{(2)}=0.07 D^{2} / \nu$, and $t^{(3)}=$ $0.09 D^{2} / \nu$. Maxima and minima are given in units of $\nu / D$. 
In a natural way, high amplitude fluctuations come along with the break of the equatorial symmetry in the velocity field. Without any symmetry constraints, the former purely antisymmetric component $\alpha_{\varphi \varphi}$ may likewise exhibit a symmetric part of either sign, see Figure 5.28 for instance. Whereas a time averaging leads to a cancellation of the symmetric contributions and results finally in a cleared antisymmetric component, variance and standard deviation are enhanced at the same time. Figure 5.27 shows the tilt of the dipole-field axis at the core-mantle boundary varying with time. The red crosses therein mark states which undergo a reversal and for which mean-field coefficients have been determined, shown in Figure 5.28. In order to distinguish reversals from erratic fluctuations it has been made sure that the actual dipole-field strength has been rebuilt at the end of each polarity transition. Excluding oscillatory solutions, temporary markedly symmetric components of the diagonal $\alpha$-components seem to be a favourable or maybe even necessary condition for axial dipole reversals to occur. While mean-field coefficients which strictly obey the symmetry rules given in Table 3.1 would preserve the parity of any initial magnetic field, irregular $\alpha$-components as presented in 5.28 allow for parity and in consequence also for polarity changes. 


\section{Two-dimensional mean-field model}

A two-dimensional axisymmetric mean-field model involving all 27 mean-field coefficients $\tilde{a}_{\kappa \lambda}$ and $\tilde{b}_{\kappa \lambda \mu}$ has been constructed in order to carry out the intended comparison between direct numerical simulations and mean-field theory. In addition, the model will help to better isolate certain dynamo processes. The model equations and the numerical techniques are briefly discussed in Section 6.1 and Section 6.2, respectively. A verification of the implemented dicretisation scheme has been performed by a comparison between analytically and numerically determined free-decay modes (see Section 6.3).

\subsection{Model equations}

By means of a decomposition of $\bar{B}$ in its poloidal and toroidal parts,

$$
\overline{\boldsymbol{B}}=\overline{\boldsymbol{B}}_{\mathrm{pol}}+\overline{\boldsymbol{B}}_{\text {tor }}
$$

with

$$
\overline{\boldsymbol{B}}_{\mathrm{pol}}=\nabla \times A \boldsymbol{e}_{\varphi}, \quad \overline{\boldsymbol{B}}_{\mathrm{tor}}=B \boldsymbol{e}_{\varphi}
$$

the dynamo equation (3.2) may be rewritten as

$$
\begin{aligned}
\partial A / \partial t= & \left(\boldsymbol{V}_{\mathrm{pol}} \times\left(\nabla \times A \boldsymbol{e}_{\varphi}\right)\right)_{\varphi}+\mathcal{E}_{\varphi}-P m^{-1}\left(\nabla \times \nabla \times A \boldsymbol{e}_{\varphi}\right)_{\varphi} \\
\partial B / \partial t= & \left(\nabla \times\left(\boldsymbol{V}_{\mathrm{pol}} \times B \boldsymbol{e}_{\varphi}+\boldsymbol{V}_{\mathrm{tor}} \times\left(\nabla \times A \boldsymbol{e}_{\varphi}\right)\right)_{\varphi}\right. \\
& +\left(\nabla \times \mathcal{E}_{\mathrm{pol}}\right)_{\varphi}-P m^{-1}\left(\nabla \times \nabla \times B \boldsymbol{e}_{\varphi}\right)_{\varphi}
\end{aligned}
$$

Here, the notations $\mathcal{E}_{\text {pol }}=\left(\mathcal{E}_{r}, \mathcal{E}_{\vartheta}, 0\right), \boldsymbol{V}_{\text {pol }}=\left(\bar{V}_{r}, \bar{V}_{\vartheta}, 0\right)$, and $\boldsymbol{V}_{\text {tor }}=\left(0,0, \bar{V}_{\varphi}\right)$ have been used. With the substitutions $a=A r \sin \vartheta, b=B r \sin \vartheta$, and $\bar{V}_{\varphi}=r \sin \vartheta \omega$, equations (6.3-6.4) may be rewritten as

$$
\begin{aligned}
\partial a / \partial t= & -\bar{V}_{r} \partial a / \partial r-\bar{V}_{\theta} / r(\partial a / \partial \theta)+r \sin \theta \mathcal{E}_{\varphi} \\
& +P m^{-1}\left(\partial^{2} a / \partial r^{2}+1 / r^{2}\left(\partial^{2} a / \partial \theta^{2}\right)-1 / r^{2} \cot \theta \partial a / \partial \theta\right) \\
\partial b / \partial t= & -b \partial \bar{V}_{r} / \partial r-\bar{V}_{r} \partial b / \partial r-b / r\left(\partial \bar{V}_{\theta} / \partial \theta\right)-\bar{V}_{\theta} / r(\partial b / \partial \theta) \\
& +b / r \cot \theta \bar{V}_{\theta}+\sin \theta((\partial \omega / \partial r)(\partial a / \partial \theta)-(\partial \omega / \partial \theta)(\partial a / \partial r)) \\
& +\sin \theta\left(r \partial \mathcal{E}_{\theta} / \partial r+\mathcal{E}_{\theta}-\partial \mathcal{E}_{r} / \partial \theta\right) \\
& +P m^{-1}\left(\partial^{2} b / \partial r^{2}+1 / r^{2}\left(\partial^{2} b / \partial \theta^{2}\right)-1 / r^{2} \cot \theta \partial b / \partial \theta\right)
\end{aligned}
$$


The components of $\mathcal{E}$ and their derivatives in (6.5-6.6) still depend on $\bar{B}$. Consequently, this dependency has to be extracted by an expansion of $\mathcal{E}$ according to

$$
\begin{aligned}
\mathcal{E}_{\kappa}= & 1 /(r \sin \vartheta)\left[\left(\tilde{a}_{\kappa \varphi}-\tilde{b}_{\kappa \varphi r} / r-\tilde{b}_{\kappa \varphi \vartheta} \cot \vartheta / r\right) b\right. \\
& +\tilde{b}_{\kappa \varphi r} \partial b / \partial r \\
& +\tilde{b}_{\kappa \varphi \vartheta} / r \partial b / \partial \vartheta \\
& +\left(\tilde{b}_{\kappa \vartheta r} / r+\tilde{b}_{\kappa \vartheta \vartheta} \cot \vartheta / r-\tilde{a}_{\kappa \vartheta}\right) \partial a / \partial r \\
& +\left(\tilde{a}_{\kappa r} / r-2 \tilde{b}_{\kappa r r} / r^{2}-\tilde{b}_{\kappa r \vartheta} \cot \vartheta / r^{2}\right) \partial a / \partial \vartheta \\
& +\left(\tilde{b}_{\kappa r r} / r-\tilde{b}_{\kappa \vartheta \vartheta} / r\right) \partial^{2} a / \partial r \partial \vartheta \\
& -\tilde{b}_{\kappa \vartheta r} \partial^{2} a / \partial r^{2} \\
& \left.+\tilde{b}_{\kappa r \vartheta} / r^{2} \partial^{2} a / \partial \vartheta^{2}\right]
\end{aligned}
$$

in which only derivatives of $\overline{\boldsymbol{B}}$ up to the first order have been considered.

Equations (6.5-6.6) are solved in a spherical shell with electrically insulating inner and outer surroundings. Thus, the magnetic field has to match a potential field $\hat{\boldsymbol{B}}$, determined by

$$
\hat{\boldsymbol{B}}_{\text {tor }}=\hat{B}=0
$$

and

$$
\nabla \times \hat{\boldsymbol{B}}_{\mathrm{pol}}=-\left(\nabla^{2}-\frac{1}{r^{2} \sin ^{2} \vartheta}\right) \hat{A}=0
$$

in both parts outside of the shell. By means of the substitutions $\hat{A}=\hat{a} /(r \sin \vartheta)$ and $\hat{B}=\hat{b} /(r \sin \vartheta)$, both equations determine likewise $a$ and $b$ in the exterior of the shell. A general solution of (6.9) rewritten in terms of $\hat{a}$ reads

$$
\hat{a}=\sum_{l=1}^{\infty} \hat{a}_{l} r^{l+1} \sin \vartheta P_{l}^{1}(\cos \vartheta)
$$

for $r \leq r_{i}$, and

$$
\hat{a}=\sum_{l=1}^{\infty} \frac{\hat{a}_{l}}{r^{l}} \sin \vartheta P_{l}^{1}(\cos \vartheta)
$$

for $r \geq r_{o}$, respectively. The $P_{l}^{1}$ are the associated Legendre polynomials, and the $\hat{a}_{l}$ are arbitrary coefficients. Thus, the boundary condition

$$
\overline{\boldsymbol{B}}=\hat{\boldsymbol{B}} \quad \text { at } r=r_{i}, r=r_{o}
$$

which is equivalent to

$$
\left(\frac{1}{r^{2} \sin \vartheta} \frac{\partial a}{\partial \vartheta},-\frac{1}{r \sin \vartheta} \frac{\partial a}{\partial r}, \frac{b}{r \sin \vartheta}\right)=\left(\frac{1}{r^{2} \sin \vartheta} \frac{\partial \hat{a}}{\partial \vartheta},-\frac{1}{r \sin \vartheta} \frac{\partial \hat{a}}{\partial r}, 0\right)
$$

at $r=r_{i}, r=r_{o}$, can be equally expressed in terms of $a$ and $b$ through

$$
a=\hat{a}, \partial a / \partial r=\partial \hat{a} / \partial r, \text { and } b=0 \quad \text { at } r=r_{i}, r=r_{o}
$$

Note that for the example of magnetoconvection, the boundary conditions for the toroidal field have been changed according to (5.1). 


\subsection{Numerical techniques}

The coupled partial differential equations (6.5-6.6) are solved by means of a finite difference method. In order to keep the difference formulas manageable, an equidistant grid in radial and latitudinal direction has been chosen. Imported variables, such as mean-field coefficients and velocity fields, evaluated on the non-equidistant radial grid points (2.1), need to be adjusted accordingly, which is done by means of a Chebyshev approximation.

An alternating direction implicit scheme for parabolic equations with mixed derivatives according to McKee et al. (1996) has been set up to discretise (6.5-6.6). This enables an efficient implicit treatment of advection and diffusion terms, while mixed and higher order derivatives are treated explicitly. In difference from McKee et al. (1996), centred difference formulas have been used whenever possible, leading to a higher accuracy. Standard difference formulas, which have been applied, are provided by Abramovich and Stegun (1965), for instance. Finally, the discretisation scheme has been found to be stable for a time step of $\Delta t=10^{-4} D^{2} / \nu$.

Note that both potentials, $A$ and $B$, as well as $\partial A / \partial r$ are forced to vanish on the polar axis, which is here included in the grid. At the same time, this compels $a, \partial a / \partial r, \partial a / \partial \vartheta$, and $b$ to vanish there.

Some attention has to be drawn to the treatment of the nonlocal boundary conditions (6.14). Let us consider at first the outer boundary at $r=r_{o}$. Moreover, a grid with $N_{r}$ radial and $N_{t}$ latitudinal grid points in increasing order is assumed. Then, $a_{j}^{N_{r}}$, denoting $a$ at the grid point $r=r_{o}, \vartheta=\vartheta_{j}$, is approximately given by

$$
a_{j}^{N r}=\sum_{l=1}^{N_{t}-2} \frac{\hat{a}_{l}}{r_{o}^{l}} \sin \vartheta_{j} P_{l}^{1}\left(\cos \vartheta_{j}\right)
$$

as prescribed by the first condition in (6.14). Adopting the summation convention, the above equation may be likewise written as

$$
a_{j}^{N_{r}}=M_{j l} \hat{a}_{l}
$$

with the quadratic coefficient matrix

$$
M_{j l}=\frac{P_{l}^{1}\left(\cos \vartheta_{j}\right)}{r_{o}^{l}} \sin \vartheta_{j}
$$

and $j=2, \cdots, N_{t}-1, l=1, \cdots, N_{t}-2$. In addition, $\partial a / \partial r$ has to be continuous at $r=r_{o}$, which requires

$$
\left.\frac{\partial a}{\partial r}\right|_{r=r_{o}} \approx \frac{3 a_{j}^{N_{r}}-4 a_{j}^{N_{r}-1}+a_{j}^{N_{r}-2}}{2 \Delta r}=\sum_{l=1}^{N_{t}-2}-l \frac{P_{l}^{1}\left(\cos \vartheta_{j}\right)}{r_{o}^{l+1}} \sin \vartheta_{j} \hat{a}_{l}
$$

Again, (6.18) is rewritten in matrix form to yield

$$
a_{j}^{N_{r}}=\tilde{M}_{j l} \hat{a}_{l}+b_{j}
$$

with

$$
\tilde{M}_{j l}=-\frac{2}{3} \Delta r \frac{l P_{l}^{1}\left(\cos \vartheta_{j}\right) \sin \vartheta_{j}}{r_{o}^{l+1}}, \quad b_{j}=\frac{4}{3} a_{j}^{N_{r}-1}-\frac{1}{3} a_{j}^{N_{r}-2}
$$


and $j=2, \cdots, N_{t}-1, l=1, \cdots, N_{t}-2$. Combining (6.16) and (6.19) finally results in

$$
a_{j}^{N_{r}}=\tilde{M}_{j l} \cdot \tilde{M}_{j l}^{-1} a_{j}^{N_{r}}+b_{j}, \quad j=2, \cdots, N_{t}-1
$$

which is equivalent to

$$
a_{j}^{N_{r}}=\left(1-\tilde{M}_{j l} M_{j l}^{-1}\right)^{-1} b_{j}, \quad j=2, \cdots, N_{t}-1
$$

For a given point in time, $a_{j}^{N_{r}}$ is determined by its values at adjacent points, $a_{j}^{N_{r}-1}$ and $a_{j}^{N_{r}-2}$. However, for the implicit treatment of radial derivatives, the following difficulty occurs: $a_{j}^{N_{r}}$ at the new point in time already needs to be known to determine $a_{j}^{N_{r}-1}$ and $a_{j}^{N_{r}-2}$. This circular dependency is resolved by an iteration method, i.e., $a_{j}^{N_{r}}$ is repeatedly assumed, and $a_{j}^{N_{r}-1}$ and $a_{j}^{N_{r}-2}$ are determined subsequently, until reasonable consistency with (6.22) is reached.

The inner boundary condition is treated in the same manner. Starting this time from

$$
a_{j}^{1}=\sum_{l=1}^{N_{t}-2} \hat{a}_{l} r^{l+1} \sin \vartheta_{j} P_{l}^{1}\left(\cos \vartheta_{j}\right)
$$

we find $a_{j}^{1}$ given by a relation corresponding to (6.22), but with

$$
M_{j l}=P_{l}^{1}\left(\cos \vartheta_{j}\right) r_{i}^{l+1} \sin \vartheta_{j}
$$

and

$$
\tilde{M}_{j l}=-\frac{2}{3} \Delta r(l+1) P_{l}^{1}\left(\cos \vartheta_{j}\right) \sin \vartheta_{j} r_{i}^{l}, \quad b_{j}=\frac{4}{3} a_{j}^{N r-1}-\frac{1}{3} a_{j}^{N r-2}
$$

instead of (6.17) and (6.20).

\subsection{Free-decay mode test}

The implementation of the discretisation scheme has been tested by a comparison between numerically and analytically determined free decay modes. The analytical derivation of the exponentially decaying field potentials $A$ and $B$ in the absence of any fluid motion is provided in the following paragraph.

The free decay of the magnetic field in the absence of any fluid motion is governed by

$$
\begin{aligned}
& \partial A / \partial t=P m^{-1}\left(\nabla^{2}-\frac{1}{r^{2} \sin ^{2} \vartheta}\right) A \\
& \partial B / \partial t=P m^{-1}\left(\nabla^{2}-\frac{1}{r^{2} \sin ^{2} \vartheta}\right) B
\end{aligned}
$$

Let us focus on the poloidal decay modes first. Inserting the ansatz

$$
A=\sum_{l} g_{l n}(r) P_{l}^{1}(\cos \vartheta) e^{-k_{l n}^{2} t}
$$


in (6.26) and setting $P m=1$, results in a Bessel equation for $g_{l n}$

$$
\left[\frac{d}{d r^{2}}+\frac{2}{r} \frac{d}{d r}+k_{l n}^{2}-\frac{l(l+1)}{r^{2}}\right] g_{l n}(r)=0
$$

which possesses the general solution

$$
g_{l n}(r)=A_{l n} j_{l}\left(k_{l n} r\right)+B_{l n} y_{l}\left(k_{l n} r\right)
$$

Here, $j_{l}\left(k_{l n} r\right)$ and $y_{l}\left(k_{l n} r\right)$ are the spherical Bessel function of first and second kind. The coefficients $A_{l n}$ and $B_{l n}$ have to be determined by the boundary conditions. The continuation of the poloidal field as a potential field in the exterior of the fluid shell requires $A$ and $\partial A / \partial r$ to be continuous at the inner and outer boundary. This results in the conditions

$$
\begin{aligned}
A_{l n} j_{l}\left(k_{l n} r_{i}\right)+B_{l n} y_{l}\left(k_{l n} r_{i}\right) & =\hat{a}_{l n} r_{i}^{l} \\
A_{l n} j_{l}^{\prime}\left(k_{l n} r_{i}\right)+B_{l n} y_{l}^{\prime}\left(k_{l n} r_{i}\right) & =\frac{l}{k_{l n}} \hat{a}_{l n} r_{i}^{l-1}
\end{aligned}
$$

at the inner boundary, and

$$
\begin{aligned}
& A_{l n} j_{l}\left(k_{l n} r_{o}\right)+B_{l n} y_{l}\left(k_{l n} r_{o}\right)=\check{a}_{l n} r_{o}^{-(l+1)} \\
& A_{l n} j_{l}^{\prime}\left(k_{l n} r_{o}\right)+B_{l n} y_{l}^{\prime}\left(k_{l n} r_{o}\right)=-\frac{(l+1)}{k_{l n}} \check{a}_{l n} r_{o}^{-(l+2)}
\end{aligned}
$$

at the outer boundary. Here, the prime means differentiation with respect to the argument. Altogether, conditions (6.31-6.34) provide a system of four linear equations for the four unknowns $A_{l n}, B_{l n}, \hat{a}_{l n}$, and $\check{a}_{l n}$. It has a nontrivial solution if, and only if

$$
\left|\begin{array}{cccc}
j_{l}\left(k_{l n} r_{i}\right) & y_{l}\left(k_{l n} r_{i}\right) & -r_{i}^{l} & 0 \\
j_{l}^{\prime}\left(k_{l n} r_{i}\right) & y_{l}^{\prime}\left(k_{l n} r_{i}\right) & -\left(l / k_{l n}\right) r_{i}^{l-1} & 0 \\
j_{l}\left(k_{l n} r_{o}\right) & y_{l}\left(k_{l n} r_{o}\right) & 0 & -r_{o}^{-(l+1)} \\
j_{l}^{\prime}\left(k_{l n} r_{o}\right) & y_{l}^{\prime}\left(k_{l n} r_{o}\right) & 0 & (l+1) / k_{l n} r_{o}^{-(l+2)}
\end{array}\right|=0
$$

By means of well known recurrence relations ${ }^{1}$, (6.35) turns out to be equivalent to

$$
j_{l+1}\left(k_{l n} r_{i}\right) y_{l-1}\left(k_{l n} r_{o}\right)-j_{l-1}\left(k_{l n} r_{o}\right) y_{l+1}\left(k_{l n} r_{i}\right)=0
$$

which serves to determine $k_{l n}(n=1,2, \cdots)$ at fixed $l$. Moreover, for fixed $l, n$, and thus $k_{l n}$, we may assume $A_{l n}=1$ without loss of generality and finally arrive at

$$
B_{l n}=\frac{r_{i}\left(k_{l n} / l\right) j_{l}^{\prime}\left(k_{l n} r_{i}\right)-j_{l}\left(k_{l n} r_{i}\right)}{y_{l}\left(k_{l n} r_{i}\right)-y_{l}^{\prime}\left(k_{l n} r_{i}\right) r_{i}\left(k_{l n} / l\right)}
$$

The toroidal decay modes have been determined in a similar way. Adopting the ansatz (6.28),

$$
B=\sum_{l} f_{l n}(r) P_{l}^{1}(\cos \vartheta) e^{-\tilde{k}_{l n}^{2} t}
$$

\footnotetext{
${ }^{1} j_{l-1}(x)+j_{l+1}(x)=(2 l+1) x^{-1} j_{l}(x), \quad(l+1) / x j_{l}(x)+j_{l}^{\prime}(x)=j_{l-1}(x)$ and equally for $y_{l}$
} 


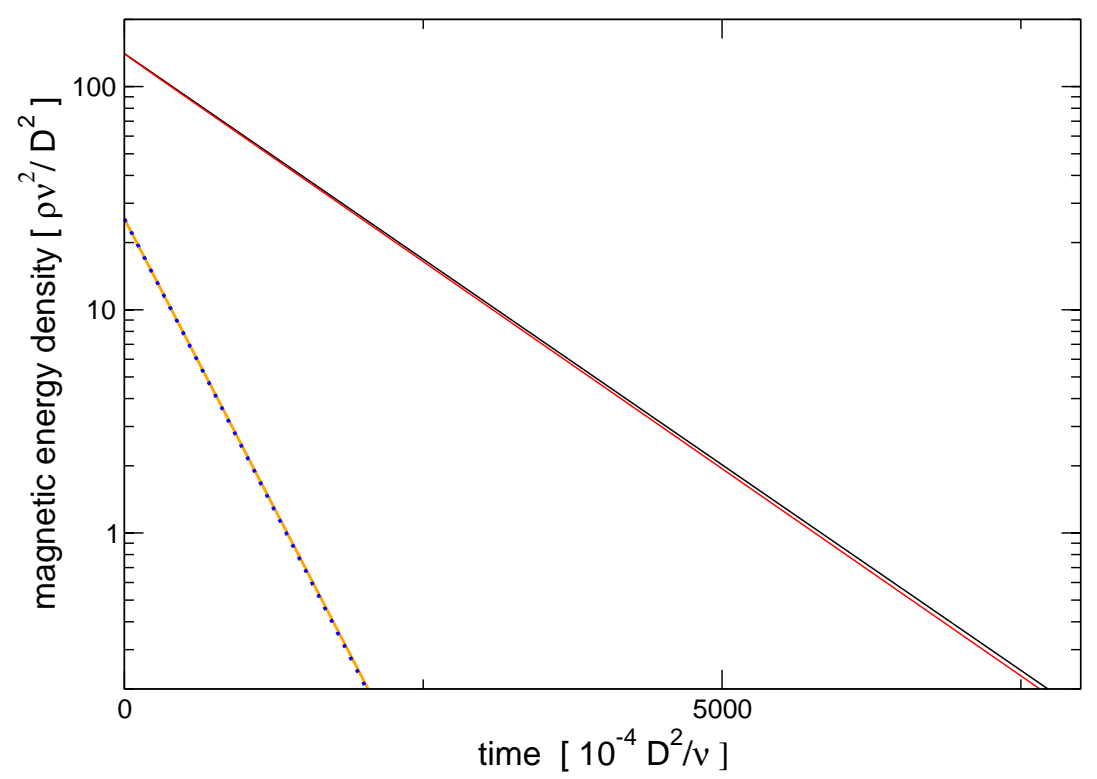

Figure 6.1: Mean magnetic energy density for the poloidal (red/black) and toroidal (blue dotted/orange) free decay modes with $l=n=1$. The decay rates are given by $k_{11}=2.06$ and $\tilde{k}_{11}=3.45$. The numerically (red/orange) and analytically (black/blue) determined curves are nearly in perfect agreement to each other.

and assuming homogeneous boundary conditions, an equation to determine $\tilde{k}_{l n}$ ( $n=$ $1,2 \cdots)$ for fixed 1 is given by

$$
j_{l}\left(\tilde{k}_{l n} r_{o}\right) y_{l}\left(\tilde{k}_{l n} r_{i}\right)-j_{l}\left(\tilde{k}_{l n} r_{i}\right) y_{l}\left(\tilde{k}_{l n} r_{o}\right)=0
$$

For fixed $l$ and $\tilde{k}_{l n}$, it is likewise possible to determine $f_{l n}$ in (6.38),

$$
f_{l n}(r)=j_{l n}\left(\tilde{k}_{l n} r\right)-\frac{j_{l n}\left(\tilde{k}_{l n} r_{i}\right)}{y_{l n}\left(\tilde{k}_{l n} r_{i}\right)} y_{l n}\left(\tilde{k}_{l n} r\right)
$$

As an example, the exponential decay of the mean magnetic energy density of the poloidal and toroidal free decay modes with $l=n=1$ is displayed in Figure 6.1. The numerically (red/orange) and analytically (black/blue dotted) determined curves are in almost perfect agreement, thus verifying the implementation of the used discretisation scheme for the diffusion operator. 


\section{Comparison between direct numerical simulations and mean-field calculations}

How well do the results given by mean-field models match with the corresponding azimuthally averaged fields determined by direct numerical simulations? If there is some match, in which way do the application of the second order correlation approximation or the assumption of isotropic 'turbulence', resulting in isotropic mean-field coefficients, effect this consistency? On the other hand, what might be plausible reasons for disagreements? These questions are tackled in Section 7.1 considering the simulation of rotating magnetoconvection (Section 5.1) and the benchmark dynamo (Section 5.2) as examples.

In Section 7.2, the action and significance of all mean-field coefficients applied is tested. At the same time, this will help to isolate and thus to identify certain dynamo processes, which constitute the axisymmetric field in these examples.

\subsection{Success of mean-field models and their limits}

The first example under consideration is the simulation of rotating magnetoconvection. Figure 7.1 presents a comparison between direct numerical simulations and mean-field calculations. In the first row, the azimuthally averaged magnetic field components resulting from a direct numerical simulation are shown. They correspond in great detail to results of our mean-field model (second row), in which all 27 mean-field coefficients have been used. The poloidal field is dipolar with inverse flux spots near the equatorial plane, and the applied azimuthal field is strongly diffused in the region occupied by the convection columns.

A mean-field simulation relying on mean-field coefficients derived in the second order correlation approximation (third row in Figure 7.1) fits equally well. This reflects that mean-field coefficients as given by SOCA, overestimated by a few per cent in their amplitudes though, still lead to a reliable parametrisation of the mean electromotive force in this parameter regime. Moreover, amplitude deviations simultaneous in $\boldsymbol{\alpha}$ and $\boldsymbol{\beta}$ might not strongly influence the efficiency of the dynamo action, as suggested by a simple scaling-analysis argument: In the mean-field description, the efficiency of dynamo action can be expressed by the magnitude of the dimensionless dynamo number, which reads $D_{a^{2}}=\alpha_{0}^{2} D^{2} / \beta_{0}^{2}$ for a presumed $\alpha^{2}$-dynamo. Here, $\alpha_{0}$ and $\beta_{0}$ mean typical scales for the $\alpha$-effect and the turbulent diffusivity, respectively, and $D$ stands for a typical length scale, e.g. the width of the spherical shell. Since $\boldsymbol{\alpha}$ and $\boldsymbol{\beta}$ are likewise overestimated in their 

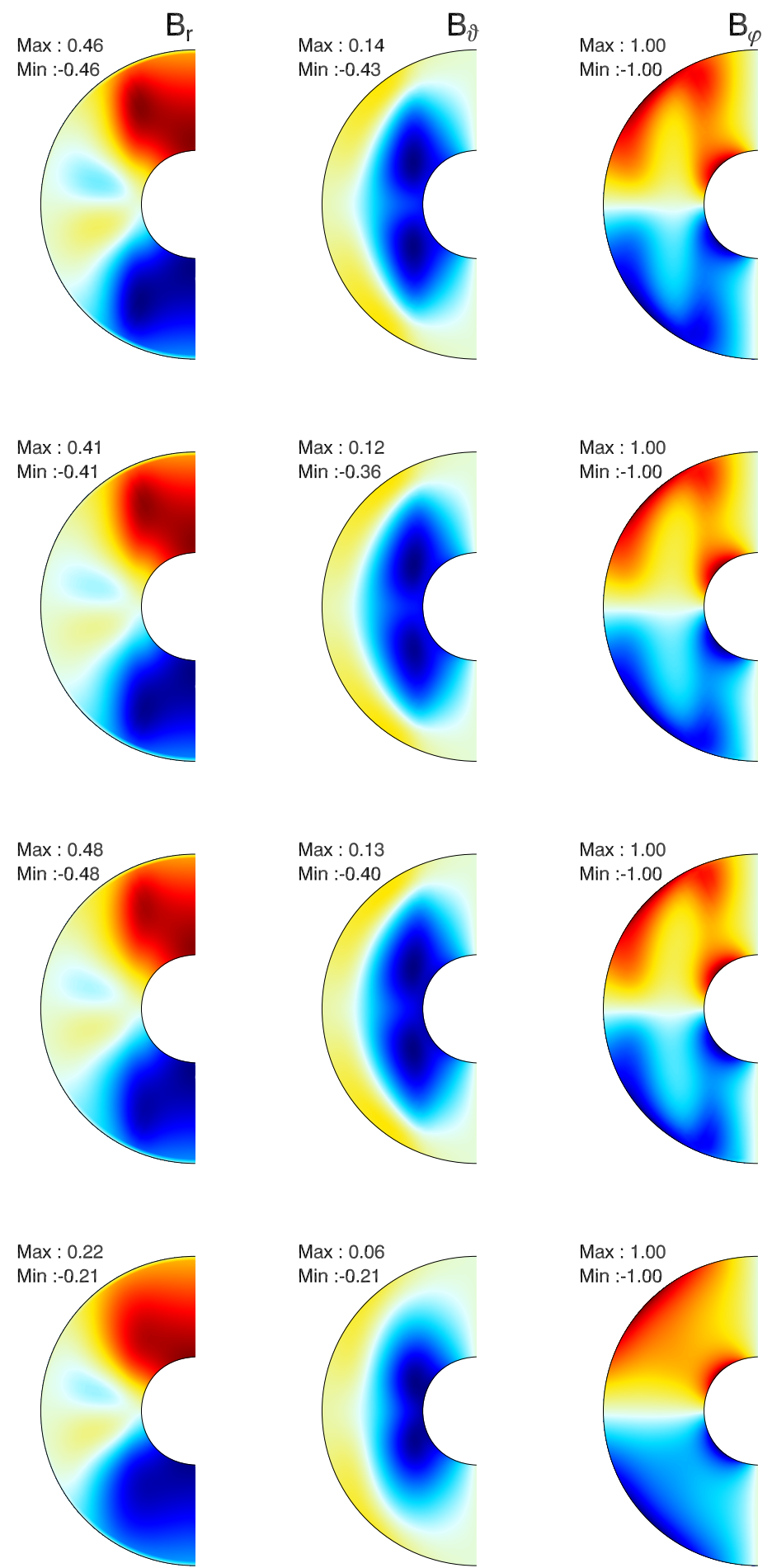

Figure 7.1: Comparison between direct numerical simulation and mean-field calculations in the example of magnetoconvection: azimuthally averaged magnetic field components resulting from a direct numerical simulation (first row), results given by the mean-field model based on a set of 27 mean-field coefficients (second row), mean-field calculation with mean-field coefficients derived applying SOCA (third row), and mean-field calculation with isotropic mean-field coefficients according to (3.43) and (3.44) (last row). Maxima and minima of the field components are given in units of $(\varrho \mu \eta \Omega)^{1 / 2}$. 
absolute amplitudes this factor cancels out and has no influence on the dynamo number. As a consequence, the resulting field resembles the mean field displayed in the second row, even though the applied mean-field coefficients had larger amplitudes.

The mean-field components shown in the last row of Figure 7.1 have been determined applying the isotropic approximations according to (3.43) and (3.44) instead of taking full tensors into account. Now, there are deviations of about $50 \%$ in the amplitudes of the poloidal field, and the toroidal field differs also in its profile; it is much less diffused at midlatitudes and mid radii where convection takes place. This difference can be clearly attributed to a missing $\gamma$-effect and in particular to the absence of $a_{r \varphi}$ and $a_{\vartheta \varphi}$ constituting $\gamma_{M T}$ as defined in (5.7). Already in the simple example considered here, the isotropic approximation fails to reproduce the axisymmetric field in satisfactory agreement with corresponding direct numerical simulations. This indicates that in general much more mean-field coefficients must be taken into account in order to grasp all relevant dynamo effects.

As a second example, the benchmark dynamo discussed in Section 5.2 has been considered. Again, in Figure 7.2, the azimuthally averaged field components resulting from a direct numerical simulation are shown in comparison with results given by mean-field modelling. Figure 7.2 is organised in the same way as Figure 7.1 before. That is, azimuthally averaged field components resulting from a direct numerical simulation have been plotted in the first row, the second row shows results obtained by corresponding mean-field calculations, the third row contributes results obtained by mean-field modelling at which the mean-field coefficients have been determined in the second order correlation approximation, while for the results presented in the last row, just the isotropic approximations (3.43) and (3.44) have been applied. Note that only the direct numerical simulation results in a steady dynamo at its critical point. All mean-field models shown in comparison are subcritical and decay according to $\overline{\boldsymbol{B}}=\overline{\boldsymbol{B}}_{0} \exp (\lambda t)$, at which $\overline{\boldsymbol{B}}_{0}$ denotes a field configuration reached after an initial transition phase, and $\lambda$ is negative in these examples. Therefore, rather decay rates than amplitudes are compared.

As in the previous example, both mean-field models relying on a set of 27 mean-field coefficients (second and third row in Figure 7.2) correspond best to the direct numerical simulation and succeed in reproducing all principal features of the axisymmetric field given in the first row. However, their agreement is not completely satisfactory. Both mean-field models are slightly subcritical with $\lambda=-0.7$ and $\lambda=-1.3$, which comes along with major topological differences in $\bar{B}_{\varphi}$ : The inverse flux bundle at low latitudes near the outer boundary is diffused too much in the mean-field models. Responsible for the high diffusivity in this region is the strong $\gamma$-effect, which leads to advection of mean toroidal field of opposite sign towards the equator, resulting in large gradients and thus in a very efficient diffusion.

Although the SOCA approximation is strictly speaking not justified anymore (see Section 5.2.4), the resulting mean field components in the third row are remarkable similar to those obtained by mean-field modelling without applying SOCA (second row). For an explanation, I refer here again to the scaling-analysis argument given above in the context of the magnetoconvection example. It is instructive to note that less reliable mean-field coefficients due to the second order correlation approximation do not effect the resulting mean-field models dramatically in these examples.

In contrast, mean-field coefficients in the isotropic approximation implemented in a 
$\mathrm{B}_{\mathrm{r}}$
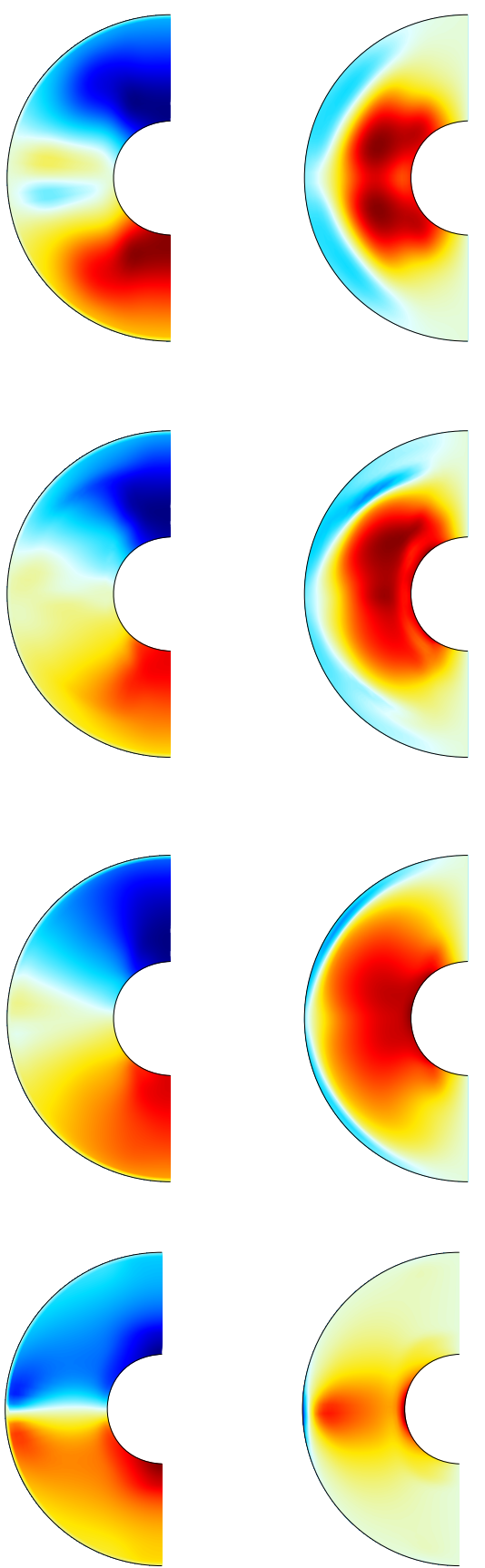

$\mathrm{B}_{\vartheta}$
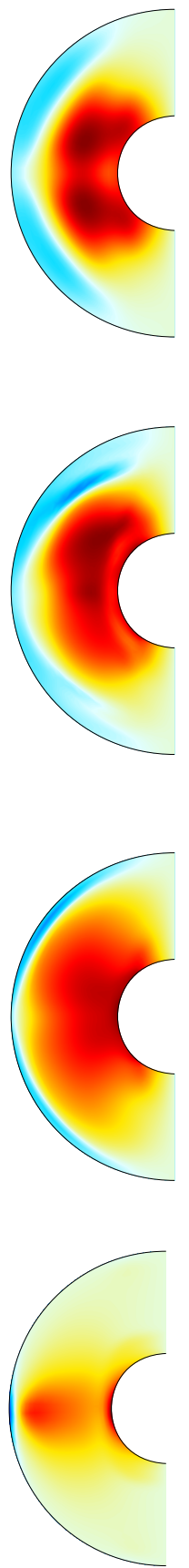

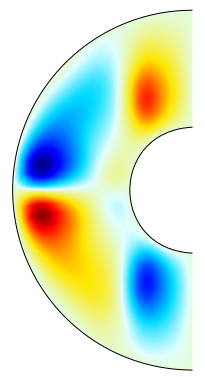

$\mathrm{B}_{\varphi}$
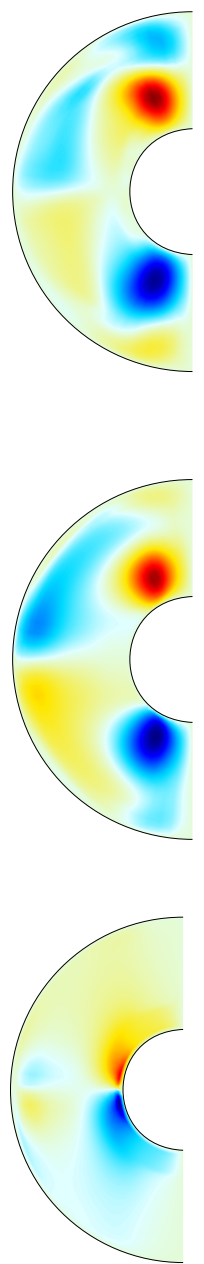

Figure 7.2: Comparison between direct numerical simulations and mean-field calculations in the example of the benchmark dynamo: azimuthally averaged magnetic field components resulting from a direct numerical simulation (first row), results as given by mean-field modelling (second row), mean-field calculation with mean-field coefficients in the second order correlation approximation (third row), or with isotropic mean-field coefficients (last row). Different from the direct numerical calculation which has become stationary, all mean-field solutions missed the critical point and decay exponentially with decay rates $\lambda=-0.7$ (second row), $\lambda=-1.3$ (third row), and $\lambda<-15$ (last row); all decay rates are given in scales of $\nu / D^{2}$. 


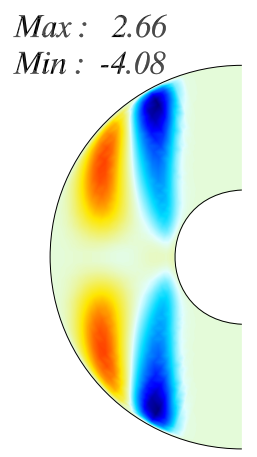

Max : 2.16

Min : -2.16

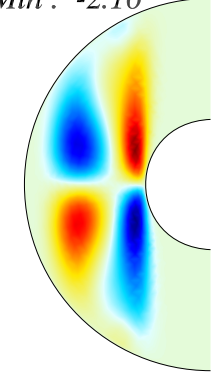

Max: 2.34

Min : -0.80

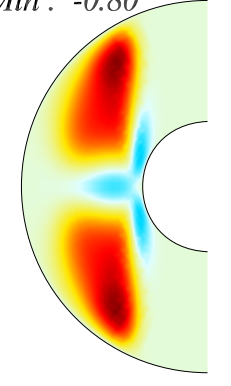

Max : 2.55

Min: -4.24

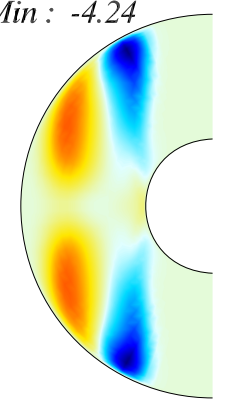

Max: 2.04

Min : -2.04

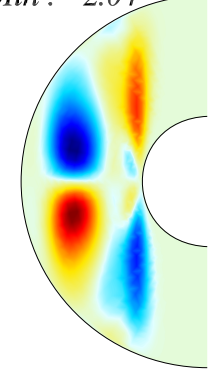

Max: 1.87

Min : -0.59

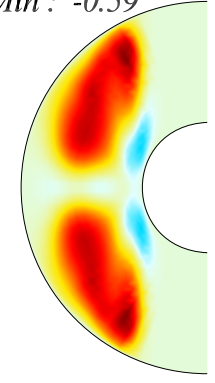

Figure 7.3: Comparison between electromotive forces in the magnetoconvection example. Left column: $\mathcal{E}_{r}^{\mathrm{DNS}}, \mathcal{E}_{\vartheta}^{\mathrm{DNS}}, \mathcal{E}_{\varphi}^{\mathrm{DNS}}$, right column: $\mathcal{E}_{r}^{(1)}, \mathcal{E}_{\vartheta}^{(1)}$, and $\mathcal{E}_{\varphi}^{(1)}$. Maxima and minima are given in units of magnetic field times velocity scaling, $\left[\nu / D(\varrho \mu \eta \Omega)^{1 / 2}\right]$.

mean-field model do not lead to reliable results anymore, see the last row in Figure 7.2. There are not only apparent differences in the field distribution, but also the decay rate, $\lambda \approx-15$, is drastically high.

The difficulties of mean-field models in accurately reproducing mean magnetic fields as determined by direct numerical simulations, which arise already in the example of the benchmark dynamo, are due to a failing parametrisation of $\mathcal{E}$. Figure 7.3 compares $\mathcal{E}^{\mathrm{DNS}}$, which refers to $\mathcal{E}$ extracted immediately from the direct numerical simulation, with $\mathcal{E}_{\lambda}^{(1)}=\tilde{a}_{\kappa \lambda} \bar{B}_{\lambda}+\tilde{b}_{\kappa \lambda r} \partial \bar{B}_{\lambda} / \partial r+1 / r \tilde{b}_{\kappa \lambda \vartheta} \partial \bar{B}_{\lambda} / \partial \vartheta$, in the example of magnetoconvection; for the computation of $\mathcal{E}^{(1)}, \bar{B}$ and its gradients have been also taken from the direct numerical simulation. Both quantities are in rather good agreement with $\delta \mathcal{E}^{(1)} \approx 0.28$, at 


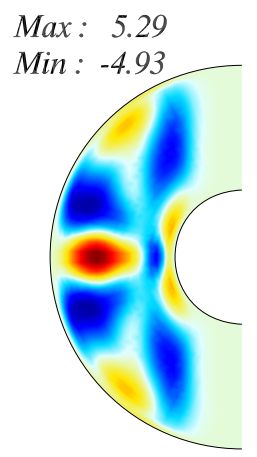

Max: 6.15

Min : -6.15

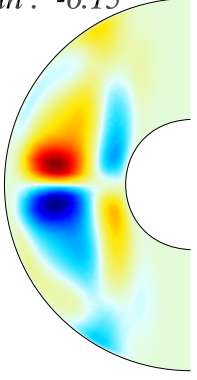

Max : 2.81

Min : -2.86

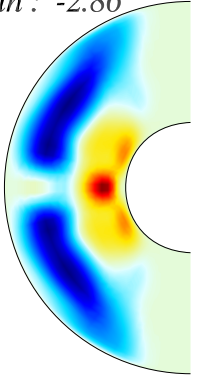

Max: 6.81

Min : -41.84

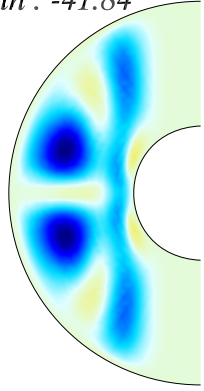

Max : 29.31

Min : -29.31

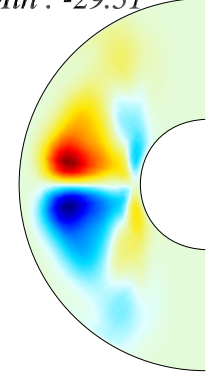

Max : 10.06

Min : -47.92

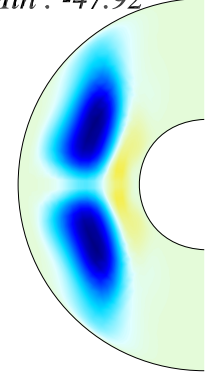

Figure 7.4: Comparison between electromotive forces in the example of the benchmark dynamo. Left column: $\mathcal{E}_{r}^{\mathrm{DNS}}, \mathcal{E}_{\vartheta}^{\mathrm{DNS}}, \mathcal{E}_{\varphi}^{\mathrm{DNS}}$, right column: $\mathcal{E}_{r}^{(0)}, \mathcal{E}_{\vartheta}^{(0)}$, and $\mathcal{E}_{\varphi}^{(0)}$. Maxima and minima are given in units of $\left[\nu / D(\varrho \mu \eta \Omega)^{1 / 2}\right]$.

which $\delta \mathcal{E}^{(1)}$ is defined by

$$
\delta \mathcal{E}^{(1)}=\frac{<\left|\mathcal{E}^{\mathrm{DNS}}-\mathcal{E}^{(1)}\right|>}{<\left|\mathcal{E}^{\mathrm{DNS}}\right|>}
$$

and $\langle\cdots\rangle$ means spatial averaging. Therefore, a parametrisation of $\mathcal{E}$ considering no higher than first-order derivatives of $\overline{\boldsymbol{B}}$ seems to be adequate in this example.

In contrast, this is not true in the example of the benchmark dynamo anymore. In this example, $\delta \mathcal{E}^{(1)}>4$ has been found, indicating that a parametrisation according to (6.7) no longer describes the actual $\mathcal{E}$ reasonably. The assumption of a scale separation, which is needed to truncate the series expansion of $\mathcal{E}$ in (3.13), breaks down. In fact, higher order derivatives of $\overline{\boldsymbol{B}}$ become large and spoil the parametrisation of $\mathcal{E}$ in a rather uncontrolled 
manner. This finding is consistent with the following observations:

- The radially averaged power spectrum of the axisymmetric magnetic field in the example of the benchmark dynamo exhibits a peak at $l=4$ containing $15 \%$ of the total power. In contrast, the corresponding spectrum in the magnetoconvection example does not possess markedly contributions for $l>2$, suggesting that the mean-field is indeed somewhat smoother in this example.

- The magnetoconvection simulation has been repeated with more complicated imposed axisymmetric fields at the boundaries in order to trigger steeper gradients in the mean field. In this way, it is indeed possible to destroy the close match between $\mathcal{E}^{\mathrm{DNS}}$ and $\mathcal{E}^{(1)}$ visible in Figure 7.3.

In the benchmark example, also coefficients $\tilde{c}_{\kappa \lambda \mu \nu}$ related to second derivatives of $\overline{\boldsymbol{B}}$ in the expansion of $\mathcal{E}$ have been taken into account, and

$$
\begin{aligned}
\mathcal{E}_{\lambda}^{(2)}= & \tilde{a}_{\kappa \lambda} \bar{B}_{\lambda}+\tilde{b}_{\kappa \lambda r} \frac{\partial \bar{B}_{\lambda}}{\partial r}+\frac{1}{r} \tilde{b}_{\kappa \lambda \vartheta} \frac{\partial \bar{B}_{\lambda}}{\partial \vartheta} \\
& +\tilde{c}_{\kappa \lambda r r} \frac{\partial^{2} \bar{B}_{\lambda}}{\partial r^{2}}+\tilde{c}_{\kappa \lambda r \vartheta} \frac{1}{r} \frac{\partial^{2} \bar{B}_{\lambda}}{\partial r \partial \vartheta}+\tilde{c}_{\kappa \lambda \vartheta \vartheta} \frac{1}{r^{2}} \frac{\partial^{2} \bar{B}_{\lambda}}{\partial \vartheta^{2}}
\end{aligned}
$$

has been considered. However, the second derivatives possess much larger absolute maxima than $\partial \bar{B}_{\lambda} / \partial r, \partial \bar{B}_{\lambda} / \partial \vartheta$, and $\mathcal{E}^{(2)}$ does not coincide superiorly with $\mathcal{E}^{\text {DNS }}$.

In Figure $7.4, \mathcal{E}^{(0)}=\tilde{\boldsymbol{a}} \overline{\boldsymbol{B}}$ is displayed in comparison with $\mathcal{E}^{\mathrm{DNS}}$. Even though the amplitudes differ markedly, the profiles provide striking similarities. This strongly suggests that despite the non-convergence of (3.13) the coefficients $\tilde{a}_{\kappa \lambda}$ in the benchmark example are meaningful.

\subsection{Action and significance of mean-field coefficients}

In order to investigate the dynamo action of certain groups of mean field coefficients, a number of test calculations has been carried out involving the following sets of mean-field coefficients:

Set I: all 27 coefficients: $\tilde{a}_{\kappa \lambda}, \tilde{b}_{\kappa \lambda r}, \tilde{b}_{\kappa \lambda \vartheta}, P m=5$

Set II: only the diagonal components $\tilde{a}_{r r}, \tilde{a}_{\vartheta \vartheta}, \tilde{a}_{\varphi \varphi}, P m=1$

Set III: $\tilde{a}_{\varphi \varphi}$ and mean flow $\overline{\boldsymbol{V}}, P m=1$

Set IV: all $\tilde{a}_{\kappa \lambda}, P m=1$

Set V: all $\tilde{a}_{\kappa \lambda}$, except $\tilde{a}_{r \varphi}, \tilde{a}_{\vartheta \varphi}, P m=1$

Set VI: all $\tilde{a}_{\kappa \lambda}$ and the coefficients $\tilde{b}_{r \varphi \vartheta}, \tilde{b}_{\vartheta \varphi r}, \tilde{b}_{\varphi \vartheta r}, \tilde{b}_{\varphi r \vartheta}$ which provide the diagonal components of the $\beta$-tensor, $P m=5$

Set VII: all $\tilde{a}_{\kappa \lambda}$ and certain $\tilde{b}_{\kappa \lambda \mu}$ which contribute to $\boldsymbol{\beta}$ and $\boldsymbol{\delta}, P m=5$ 


\begin{tabular}{|c|c|r|}
\hline \multicolumn{3}{|c|}{ BENCHMARK DYNAMO } \\
\hline \hline SET & $\lambda_{A}$ & \multicolumn{1}{|c|}{$\lambda_{S}$} \\
\hline I & -0.9 & -8.1 \\
II & +4.8 & +3.3 \\
III & -9.0 & -16.0 \\
IV & -2.2 & -8.9 \\
V & -0.4 & 2.3 \\
VI & -3.1 & -12.0 \\
VII & -0.6 & -5.7 \\
\hline
\end{tabular}

Table 7.1: Exponential decay or growth rates in units of $\nu / D^{2}$ for equatorial symmetric $\left(\lambda_{A}\right)$ and antisymmetric $\left(\lambda_{S}\right)$ mean-field solutions involving various sets of mean-field coefficients.

Set I includes all 27 coefficients $\tilde{a}_{\kappa \lambda}, \tilde{b}_{\kappa \lambda \mu}$, i.e. $\boldsymbol{\alpha}, \boldsymbol{\gamma}, \boldsymbol{\beta}, \boldsymbol{\delta}$, and $\boldsymbol{\kappa}$. Therefore, mean-field calculations involving Set I serve as a reference state. Set II and III have been chosen in order to test the dynamo action of the diagonal components of the $\alpha$-tensor, whereas Set IV and V tend to demonstrate the influence of certain non-diagonal components, in particular the action of the $\gamma$-effect. The last two Sets, VI and VII, have been chosen in order to reveal the importance of further coefficients $\tilde{b}_{\kappa \lambda \mu}$ which provide the $\beta$-tensor and the $\delta$-vector.

In the example of the benchmark dynamo, all mean-field solutions based on the above defined sets are either exponentially growing or decaying after an initial transition period. Table 7.1 gives the decay or growth rates. Because $E^{S}$ and $E^{A}$ solutions are independent of each other, it is justified to determine their growth rates separately by prescribing equatorial symmetry conditions.

The benchmark dynamo operates through an $\alpha^{2}$-mechanism. Mean-field calculations involving Set II result in similar growth rates $\lambda_{A}=4.8$ and $\lambda_{S}=3.3$ for the $E^{A}$ and $E^{S}$
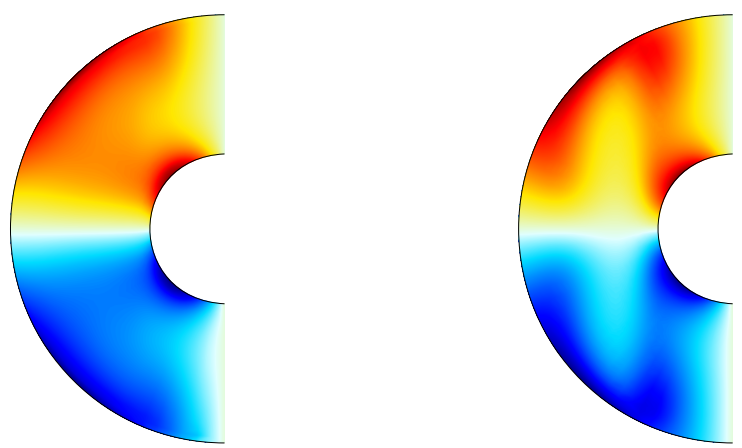

Figure 7.5: Mean azimuthal field in the magnetoconvection example as given by meanfield modelling, involving all mean-field coefficients (right chart) and without $\tilde{a}_{r \varphi}, \tilde{a}_{\vartheta \varphi}$ (left chart). 

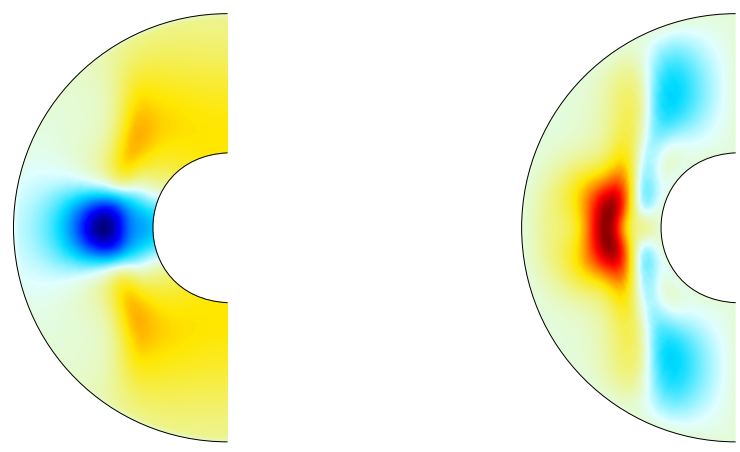

Figure 7.6: $\bar{B}_{r}$ (left contour plot) and $\bar{B}_{\varphi}$ (right contour plot) as given by mean-field calculations for the benchmark dynamo involving Set V.

solution, respectively. Since the differential rotation is negligible, an attempt to close the dynamo cycle by an $\omega$-effect fails, see Set III in Table 7.1.

Besides the diagonal components, $\tilde{a}_{r \varphi}$ and $\tilde{a}_{\vartheta \varphi}$ are most important. They provide a strong $\gamma$-effect, which acts to expel flux from the central dynamo region. This effect is clearly visible in Figure 7.5, which refers to the magnetoconvection example. Moreover, again in the benchmark example, the absence of $\tilde{a}_{r \varphi}$, and $\tilde{a}_{\vartheta \varphi}$ in Set V allows for a supercritical $E^{S}$ solution $\left(\lambda_{S}=2.3\right)$ while an equatorial symmetric azimuthal field decays rapidly if these non-diagonal components are involved (Set IV, $\lambda_{S}=-8.9$ ). Figure 7.6 shows $\bar{B}_{r}$ and $\bar{B}_{\varphi}$ given by mean-field calculations which rely on mean-field coefficients combined in Set V, i.e. without $\tilde{a}_{r \varphi}$ and $\tilde{a}_{\vartheta \varphi}$. Both components show strong flux concentrations around the equatorial plane at mid radii, and it is plausible that these solutions become unfavourable under the influence of a strong $\gamma$-effect. Together with the turbulent diffusion, which is also concentrated in the region covered by the convection columns (see e.g. Figure 5.7), the $\gamma$-effect leads to a clear preference of $E^{A}$ solutions.

With the diagonal components of the $\beta$-tensor taken into account (Set VI), diffusion is significantly enhanced, and the resulting dynamo solutions are markedly subcritical. However, not all components of $\tilde{\boldsymbol{b}}$ are conducive to the turbulent diffusion. If in addition all coefficients $\tilde{b}_{\kappa \lambda \mu}$ which contribute to the $\delta$-vector are considered (Set VII), the decay rates increase, and the corresponding $E^{A}$-solution gets close to the critical point. This is consistent with the idea of a constructive $\delta \times j$-effect due to an anisotropic turbulent conductivity (see e.g. Rädler 1980).

Altogether, the test calculations suggest a minimum set of 17 mean-field coefficients which should be considered in a mean-field model in order to achieve reasonable agreement between mean-field simulations and direct numerical simulations. These are: the diagonal components $\tilde{a}_{r r}, \tilde{a}_{\vartheta \vartheta}, \tilde{a}_{\varphi \varphi}$, the dominating non-diagonal components $\tilde{a}_{r \varphi}, \tilde{a}_{\vartheta \varphi}$, and all components $\tilde{b}_{\kappa \lambda \mu}$ except for $\tilde{b}_{\kappa r r}$ and $\tilde{b}_{\kappa \vartheta \vartheta}$. They contribute to the $\alpha$-effect, the $\gamma$-effect, the turbulent diffusion, and the $\boldsymbol{\delta} \times \boldsymbol{j}$-effect, which thus can be identified as the relevant dynamo mechanisms in the benchmark example. 



\section{Conclusions and outlook}

The knowledge of relevant mean-field coefficients is decisive in order to analyse and to model dynamo action in many astrophysical circumstances. In this work, two approaches to determine mean-field coefficients have been developed. Approach (I) is based on a numerical determination of electromotive forces, or more precisely, on numerical solutions of the induction equation for the residual field. But otherwise, it is completely general. Thus, it can be easily applied to a variety of dynamo simulations. Approach (II) requires certain simplifications, namely the assumption of the velocity field being solenoidal and steady, the second order correlation approximation, and the neglect of the mean flow. On the other hand, it reproduces the mean-field coefficients for an azimuthal average in the low-conductivity limit if solely an appropriate velocity field is provided.

Approach (I) has been applied to four examples: a simulation of rotating magnetoconvection, a quasi-stationary dynamo, and two highly time dependent dynamos, at which one of them shows dipole reversals. Despite their differences in the vigour of convection or in the magnetic diffusivity, the resulting (time-averaged) mean-field coefficients provide striking similarities. This implies that in all four examples similar dynamo processes take place, which appear to be typical features of columnar convection inside a sphere or a spherical shell. These are: the generation of poloidal and toroidal magnetic field by an $\alpha$-effect, flux expulsion from the dynamo region due to a $\gamma$-effect, and a strong turbulent diffusion, which might be moderated by a $\delta \times \boldsymbol{j}$-effect.

Furthermore, the calculation of mean-field coefficients provides some insight into the reliability of frequently applied approximations in the framework of mean-field theory. Most dubious among them is the reduction of the $\alpha$-tensor to an isotropic tensor, which leaves dominating non-diagonal components unconsidered. A further, important simplification is the second order correlation approximation. It typically leads to overestimated amplidudes of mean-field coefficients, whereas their profiles are rather unaffected.

The mean-field picture of geodynamo models is completed by the simulation of axisymmetric fields by means of a mean-field model, involving all mean-field coefficients determined. Various test calculations with different sets of mean-field coefficients confirm their relation to the above mentioned dynamo processes. In addition, a comparison with azimuthally averaged fields resulting from direct numerical simulations reveals their significance. In the magnetoconvection and the benchmark example considered here, the match between direct numerical simulations and mean-field simulations is best if at least a number of 17 mean-field coefficients are involved which contribute to $\boldsymbol{\alpha}, \boldsymbol{\gamma}, \boldsymbol{\beta}$, and $\delta$. The application of corresponding mean-field coefficients derived in the second order correlation approximation leads to very similar results.

The reliability of mean-field models relies on a proper parametrisation of $\mathcal{E}$ in terms of the mean magnetic field. In the simple magnetoconvection example, the traditional repre- 
sentation of the mean electromotive force considering no higher than first order derivatives of the mean magnetic field is valid. However, already in the benchmark example, it seems to be no longer justified. The regard of second order derivatives in addition does not improve the parametrisation indicating that the assumption of scale separation is not fulfilled. This defect severely limits the applicability of mean-field theory. Nonetheless, the axisymmetric field obtained by mean-field modelling in the benchmark example matches the corresponding direct numerical simulation surprisingly well.

Here, only steady mean-field coefficients have been used in mean-field models. In a future project, it will be interesting to carry out mean-field calculations which involve also time-dependent mean-field coefficients. This may provide some insight into interesting time-dependent characteristics of geodynamo models, such as dipole reversals and excursions. Do mean-field models exhibit reversals if heavily fluctuating mean-field coefficients are involved? Are diagonal components of $\boldsymbol{\alpha}$ which possess an equatorial symmetric part, as presented in Figure 5.28, conducive to reversals? In order to tackle these questions, a massive computation of time sequences of mean-field coefficients is required, which can be subsequently used in mean-field models. Alternatively, one could rely on time-averaged mean-field coefficients and parametrise the time fluctuations. The parametrisation should involve the standard deviation as well as spatial and temporal scales on which fluctuations occur. First steps following this line have been performed in Sections 5.3 and 5.4, in which the time variability of mean-field coefficients has been analysed in two examples.

In order to extrapolate results for mean-field coefficients and to apply them to dynamo simulations in different astrophysical contexts, scaling laws for mean-field coefficients are needed. How does the $\alpha$-tensor, for instance, depend on the vigour of convection or on the rotation rate? In which way are $\alpha$-components quenched due to the presence of a mean field? Starting with an extensive, systematic parameter study, it may be possible to derive scaling laws, which reveal the dependence of some mean-field coefficients on key parameters as the magnetic Reynolds number, the Rossby number or the Elsasser number. Therefore, calculations as performed in Section 5.1.4, giving $\left(\alpha_{\varphi \varphi}\right)_{\mathrm{rms}}$ in dependence of $R m$, or in Section 5.2.4, giving $\left(\alpha_{\varphi \varphi}\right)_{\mathrm{rms}}$ in dependence of $\Lambda$ have to be extended to a wider parameter space. The numerical survey can be assisted by a generalisation of approach (II) to the case of non-steady motions, which will lead to useful constraints: the scaling laws have to be consistent with resulting analytical expressions in parameter regimes in which SOCA is justified. However, a generalisation of approach (II) is algebraically involved and computationally expensive. In the non-steady case, the expressions for $S_{l}^{m}$ and $T_{l}^{m}$ in (4.30-4.31) have to be replaced by solutions of

$$
D_{l} S_{l}^{m}=F_{l}^{m}, \quad D_{l} T_{l}^{m}=G_{l}^{m}, \quad r_{i}<r<r_{a}
$$

at which $D_{l}$ is defined as

$$
D_{l} f=\frac{1}{r} \frac{\partial^{2}}{\partial r^{2}}(r f)-\frac{l(l+1)}{r^{2}} f-\frac{1}{\eta} \frac{\partial f}{\partial t}
$$

and all other notations as well as the boundary conditions are the same as in Section 4.2. Again, the solutions of (8.1) can be given with the help of certain Green's functions $g_{l}$ 
and $h_{l}$. But, these are now time dependent, and an additional time integration is needed,

$$
\begin{aligned}
S_{l}^{m}(r, t)= & -\int_{t_{0}}^{t} \int_{r_{i}}^{r_{o}} g_{l}\left(r, r^{\prime}, t-t^{\prime}\right) F_{l}^{m}\left(r^{\prime}, t^{\prime}\right) d r^{\prime} d t^{\prime} \\
& +\int_{r_{i}}^{r_{o}} g_{l}\left(r, r^{\prime}, t-t_{0}\right) S_{l}^{m}\left(r^{\prime}, t_{0}\right) d r^{\prime} \\
T_{l}^{m}(r, t)= & -\int_{t_{0}}^{t} \int_{r_{i}}^{r_{o}} h_{l}\left(r, r^{\prime}, t-t^{\prime}\right) G_{l}^{m}\left(r^{\prime}, t^{\prime}\right) d r^{\prime} d t^{\prime} \\
& +\int_{r_{i}}^{r_{o}} h_{l}\left(r, r^{\prime}, t-t_{0}\right) T_{l}^{m}\left(r^{\prime}, t_{0}\right) d r^{\prime}
\end{aligned}
$$

In the above equations, $t_{0}$ means the initial time. The single integrals over $r^{\prime}$ contributing to $S_{l}^{m}$ and $T_{l}^{m}$ are just solutions of the homogeneous equations (8.1), while the double integrations are solutions of the inhomogeneous equations vanishing at $t=t_{0}$. With (8.38.4) instead of (4.30-4.31), all relations in Section 4.2 which are based on (4.30-4.31) have to be changed accordingly.

Approach (I) developed in this work serves equally well to determine mean-field coefficients for any hydromagnetic flow. Besides the parameter study suggested above, it will be interesting to apply approach (I) to global anelastic dynamo simulations, dedicated to simulate stellar dynamos (e.g. Brun et al. 2004), or to fully compressible box simulations, which are performed to simulate turbulence in local sections of the solar convection zone (e.g. Brandenburg et al. 1996, Ossendrijver et al. 2002), in the galactic disc (e.g. Ziegler et al. 1996), or in accretion discs (Brandenburg et al. 1995, Hawley et al. 1996). This analysis will highlight specific properties of mean-field coefficients due to a density stratification and a changed geometry of the flow domain. 



\section{A Relations between covariant and non-covariant mean-field coefficients}

A set of covariant mean-field coefficients specified for $\tilde{b}_{\kappa \lambda \varphi}=0$ reads:

$$
\begin{aligned}
\alpha_{r r} & =-\left(\tilde{a}_{r r}-\tilde{b}_{r \vartheta \vartheta} / r\right) \\
\alpha_{r \vartheta} & =\alpha_{\vartheta r}=-1 / 2\left(\tilde{a}_{r \vartheta}+\tilde{b}_{r r \vartheta} / r+\tilde{a}_{\vartheta r}-\tilde{b}_{\vartheta \vartheta \vartheta} / r\right) \\
\alpha_{r \varphi} & =\alpha_{\varphi r}=-1 / 2\left(\tilde{a}_{r \varphi}+\tilde{a}_{\varphi r}-\tilde{b}_{\varphi \vartheta \vartheta} / r\right) \\
\alpha_{\vartheta \vartheta} & =-\left(\tilde{a}_{\vartheta \vartheta}+\tilde{b}_{\vartheta r \vartheta} / r\right) \\
\alpha_{\vartheta \varphi} & =\alpha_{\varphi \vartheta}=-1 / 2\left(\tilde{a}_{\vartheta \varphi}+\tilde{a}_{\varphi \vartheta}+\tilde{b}_{\varphi r \vartheta}\right) \\
\alpha_{\varphi \varphi} & =\tilde{a}_{\varphi \varphi} \\
\beta_{r r} & =-1 / 2 \tilde{b}_{r \varphi \vartheta} \\
\beta_{r \vartheta} & =\beta_{\vartheta r}=1 / 4\left(\tilde{b}_{r \varphi r}-\tilde{b}_{\vartheta \varphi \vartheta}\right) \\
\beta_{r \varphi} & =\beta_{\varphi r}=1 / 4\left(\tilde{b}_{r r \vartheta}-\tilde{b}_{\varphi \varphi \vartheta}-\tilde{b}_{r \vartheta r}\right) \\
\beta_{\vartheta \vartheta} & =1 / 2 \tilde{b}_{\vartheta \varphi r} \\
\beta_{\vartheta \varphi} & =\beta_{\varphi \vartheta}=1 / 4\left(\tilde{b}_{\vartheta r \vartheta}+\tilde{b}_{\varphi \varphi r}-\tilde{b}_{\vartheta \vartheta r}\right) \\
\beta_{\varphi \varphi} & =1 / 2\left(\tilde{b}_{\varphi r \vartheta}-\tilde{b}_{\varphi \vartheta r}\right) \\
\gamma_{r} & =1 / 2\left(\tilde{a}_{\vartheta \varphi}-\tilde{a}_{\varphi \vartheta}-\tilde{b}_{\varphi r \vartheta} / r\right) \\
\gamma_{\vartheta} & =1 / 2\left(\tilde{a}_{\phi r}-\tilde{b}_{\varphi \vartheta \vartheta} / r-\tilde{a}_{r \varphi}\right) \\
\gamma_{\varphi} & =1 / 2\left(\tilde{a}_{r \vartheta}+\tilde{b}_{r r \vartheta} / r-\tilde{a}_{\vartheta r}+\tilde{b}_{\vartheta \vartheta \vartheta} / r\right) \\
\delta_{r} & =1 / 4\left(\tilde{b}_{\vartheta \vartheta r}-\tilde{b}_{\vartheta r \vartheta}+\tilde{b}_{\varphi \varphi r}\right) \\
\delta_{\vartheta} & =1 / 4\left(\tilde{b}_{r r \vartheta}-\tilde{b}_{r \vartheta r}+\tilde{b}_{\varphi \varphi r}\right) \\
\delta_{\varphi} & =-1 / 4\left(\tilde{b}_{r \varphi r}+\tilde{b}_{\vartheta \varphi \vartheta}\right) \\
&
\end{aligned}
$$




$$
\begin{aligned}
\kappa_{\lambda r r} & =-\tilde{b}_{\lambda r r} \\
\kappa_{\lambda r \vartheta} & =\kappa_{\lambda \vartheta r}=-1 / 2\left(\tilde{b}_{\lambda r \vartheta}+\tilde{b}_{\lambda \vartheta r}\right) \\
\kappa_{\lambda r \varphi} & ==\kappa_{\lambda \varphi r}=-1 / 2 \tilde{b}_{\lambda \varphi r} \\
\kappa_{\lambda \vartheta \vartheta} & =-\tilde{b}_{\lambda \vartheta \vartheta} \\
\kappa_{\lambda \vartheta \varphi} & =\kappa_{\lambda \varphi \vartheta}=-1 / 2 \tilde{b}_{\lambda \varphi \vartheta} \\
\kappa_{\lambda \varphi \varphi} & =0
\end{aligned}
$$




\section{B Representation of a second rank tensor which depends on two directions}

Consider a second-rank tensor $a_{i j}$ depending on two unit vectors $\hat{\boldsymbol{p}}$ and $\hat{\boldsymbol{r}}$. A multiplication with two auxiliary vectors $\boldsymbol{v}$ and $\boldsymbol{w}$ determines $a_{i j} v_{i} w_{j}$ as a function of $\boldsymbol{v}, \boldsymbol{w}, \hat{\boldsymbol{p}}$ and $\hat{\boldsymbol{r}}$

$$
\boldsymbol{v}^{T} \boldsymbol{a} \boldsymbol{w}=a_{i j} v_{i} w_{j}=f(\boldsymbol{v}, \boldsymbol{w}, \hat{\boldsymbol{p}}, \hat{\boldsymbol{r}})
$$

Because $a_{i j} v_{i} w_{j}$ is a scalar quantity, $f(\boldsymbol{v}, \boldsymbol{w}, \hat{\boldsymbol{p}}, \hat{\boldsymbol{r}})$ has to be inferred from independent scalar combinations (i.e. irreducible invariants) of $\boldsymbol{v}, \boldsymbol{w}, \hat{\boldsymbol{p}}$ and $\hat{\boldsymbol{r}}$. As a further restriction, all relevant invariants have to be bilinear in $\boldsymbol{v}$ and $\boldsymbol{w}$. All irreducible invariants are given then by

$$
\begin{gathered}
v_{i} w_{i}, \epsilon_{i j k} v_{i} w_{j} \hat{r}_{k}, \epsilon_{i j k} v_{i} w_{j} \hat{p}_{k}, \epsilon_{i j k} v_{i} w_{j}(\hat{\boldsymbol{p}} \times \hat{\boldsymbol{r}})_{k} \\
v_{i} \hat{r}_{i} w_{j} \hat{r}_{j}, v_{i} w_{j}\left(\hat{p}_{i} \hat{r}_{j}+\hat{p}_{j} \hat{r}_{i}\right), v_{i} \hat{p}_{i} w_{j} \hat{p}_{j} \\
v_{i} w_{j}\left[\hat{r}_{i}(\hat{\boldsymbol{p}} \times \hat{\boldsymbol{r}})_{j}+\hat{r}_{j}(\hat{\boldsymbol{p}} \times \hat{\boldsymbol{r}})_{i}\right], v_{i} w_{j}\left[\hat{p}_{i}(\hat{\boldsymbol{p}} \times \hat{\boldsymbol{r}})_{j}+\hat{p}_{j}(\hat{\boldsymbol{p}} \times \hat{\boldsymbol{r}})_{i}\right]
\end{gathered}
$$

Note that the product of $\epsilon_{i j k} v_{i} \hat{r}_{j} \hat{p}_{k}$ and $\epsilon_{i j k} w_{i} \hat{r}_{j} \hat{p}_{k}$ need not be taken into account, because it is found to be reducible:

$$
\begin{aligned}
\epsilon_{i j k} v_{i} \hat{r}_{j} \hat{p}_{k} \epsilon_{l m n} w_{l} \hat{r}_{m} \hat{p}_{n}=\delta_{l m n}^{i j k} v_{i} w_{l} \hat{r}_{j} \hat{r}_{m} \hat{p}_{k} \hat{p}_{n}= & v_{i} w_{i}+v_{i} w_{j}\left(\hat{r}_{i} \hat{p}_{j}+\hat{r}_{j} \hat{p}_{i}\right) \hat{p}_{k} \hat{r}_{k} \\
& -v_{i} \hat{r}_{i} w_{j} \hat{r}_{j}-v_{i} \hat{p}_{i} w_{j} \hat{p}_{j}-v_{i} w_{i}\left(\hat{p}_{j} \hat{r}_{j}\right)^{2}
\end{aligned}
$$

at which the symbol $\delta_{l m n}^{i j k}$ is defined as

$$
\delta_{l m n}^{i j k}=\delta_{i l} \delta_{j m} \delta_{k n}+\delta_{i m} \delta_{j n} \delta_{k l}+\delta_{i n} \delta_{j l} \delta_{k m}-\delta_{i m} \delta_{j l} \delta_{k n}-\delta_{i l} \delta_{j n} \delta_{k m}-\delta_{i n} \delta_{j m} \delta_{k l}
$$

Thus, (B.1) can be represented as a linear combination of all terms listed in (B.2):

$$
\begin{aligned}
a_{i j} v_{i} w_{j}= & a_{1} v_{i} w_{j} \delta_{i j}+a_{2} v_{i} w_{j} \hat{r}_{i} \hat{r}_{j}+a_{3} v_{i} w_{j} \hat{p}_{i} \hat{p}_{j}+a_{4} v_{i} w_{j}\left(\hat{r}_{i} \hat{p}_{j}+\hat{r}_{j} \hat{p}_{i}\right) \\
& +a_{5} v_{i} w_{j}\left[\hat{r}_{i}(\hat{\boldsymbol{p}} \times \hat{\boldsymbol{r}})_{j}+\hat{r}_{j}(\hat{\boldsymbol{p}} \times \hat{\boldsymbol{r}})_{i}\right] \\
& +a_{6} v_{i} w_{j}\left[\hat{p}_{i}(\hat{\boldsymbol{p}} \times \hat{\boldsymbol{r}})_{j}+\hat{p}_{j}(\hat{\boldsymbol{p}} \times \hat{\boldsymbol{r}})_{i}\right]+a_{7} \epsilon_{i j k} v_{i} w_{j} \hat{r}_{k} \\
& +a_{8} \epsilon_{i j k} v_{i} w_{j} \hat{p}_{k}+a_{9} \epsilon_{i j k} v_{i} w_{j}(\hat{\boldsymbol{p}} \times \hat{\boldsymbol{r}})_{k}
\end{aligned}
$$

All coefficients $a_{1}, \cdots, a_{9}$ may be any functions of those invariants which are independent of $\boldsymbol{v}$ and $\boldsymbol{w}$, that is $a_{i}=a_{i}(\hat{\boldsymbol{p}} \hat{\boldsymbol{r}}), i=1, \cdots, 9$, for the example considered here. Because (B.3) holds for any vectors $\boldsymbol{v}$ and $\boldsymbol{w}$, the general representations (3.45) and (3.46) are conclusive. 



\section{Green's functions and orthogonality relations}

The Green's functions in (4.30-4.31) are given by

$$
f_{l}\left(r, r^{\prime}\right)= \begin{cases}\frac{1}{(2 l+1) r}\left(\frac{r^{\prime}}{r}\right)^{l}, & r^{\prime} \leq r \\ \frac{1}{(2 l+1) r}\left(\frac{r}{r^{\prime}}\right)^{l+1}, & r^{\prime} \geq r^{\prime}\end{cases}
$$

and

$$
g_{l}\left(r, r^{\prime}\right)=\left\{\begin{array}{c}
\left(1-\left(\frac{r_{i}}{r_{o}}\right)^{2 l+1}\right)^{-1}\left[f_{l}\left(r, r^{\prime}\right)-\left(\frac{r}{r_{o}}\right)^{l} f_{l}\left(r_{o}, r^{\prime}\right)\right. \\
\left.-\left(\frac{r_{i}}{r}\right)^{l+1} f_{l}\left(r_{i}, r^{\prime}\right)+\frac{r^{l} r_{i}^{l+1}}{r_{o}^{2 l+1}} f_{l}\left(r_{i}, r^{\prime}\right)\right], \quad r^{\prime} \leq r \\
\left(1-\left(\frac{r}{r_{o}}\right)^{2 l+1}\right)^{-1}\left[f_{l}\left(r, r^{\prime}\right)-\left(\frac{r}{r_{o}}\right)^{l} f_{l}\left(r_{o}, r^{\prime}\right)\right. \\
\left.-\left(\frac{r_{i}}{r}\right)^{l+1} f_{l}\left(r_{i}, r^{\prime}\right)+\frac{r_{i}^{2 l+1}}{r^{l+1} r_{o}^{l}} f_{l}\left(r_{o}, r^{\prime}\right)\right], \quad r^{\prime} \geq r
\end{array}\right.
$$

We note that

$$
\frac{\partial f_{l}\left(r, r^{\prime}\right)}{\partial r}-\frac{l}{r} f_{l}\left(r, r^{\prime}\right)=g_{l}\left(r, r^{\prime}\right)=0 \quad \text { at } \quad r=r_{i}
$$

and

$$
\frac{\partial f_{l}\left(r, r^{\prime}\right)}{\partial r}+\frac{l+1}{r} f_{l}\left(r, r^{\prime}\right)=g_{l}\left(r, r^{\prime}\right)=0 \quad \text { at } \quad r=r_{o}
$$

The orthogonality relations used to evaluate (4.37) are given by

$$
\begin{aligned}
& \int_{0}^{\pi} P_{l^{\prime} l}^{m}(\vartheta) \sin \vartheta \mathrm{d} \vartheta=\frac{2(l+|m|) !}{(2 l+1)(l-|m|) !} \delta_{l^{\prime} l} \\
& \int_{0}^{\pi}\left(Q_{l^{\prime} l}^{m}(\vartheta)+Q_{l l^{\prime}}^{m}(\vartheta)\right) \mathrm{d} \vartheta=0 \\
& \int_{0}^{\pi} R_{l^{\prime} l}^{m}(\vartheta) \sin \vartheta \mathrm{d} \vartheta=\frac{2 l(l+1)(l+|m|) !}{(2 l+1)(l-|m|) !} \delta_{l^{\prime} l}
\end{aligned}
$$





\section{Components of $\tilde{a}$ in the second order correlation approximation}

In the following, all coefficients of $\tilde{\boldsymbol{a}}$ determined by means of approach (II) are given. For $\tilde{a}_{\vartheta \lambda}$ and $\tilde{a}_{\varphi \lambda}$ an additional numerical integration over $\vartheta^{\prime}$ is needed because the orthogonality relations (C.3-C.5) can not be applied in a similar way. A rather high resolution of $l_{\max }=85$ and 65 grid points in radial direction requires a parallelisation of the computation.

$$
\begin{aligned}
\tilde{a}_{r r}(r, \vartheta)= & \frac{2}{\eta} \sum_{l, l^{\prime} ; m>0}\left\{\int _ { 0 } ^ { R } \left(\hat{f}_{l}\left(r, r^{\prime}\right) \operatorname{Re}\left(\psi_{l^{\prime}}^{m *}(r) \hat{\phi}_{l}^{m}\left(r^{\prime}\right)\right)\right.\right. \\
& \left.+\tilde{g}_{l}\left(r, r^{\prime}\right) \operatorname{Re}\left(\hat{\phi}_{l^{\prime}}^{m *}(r) \psi_{l}^{m}\left(r^{\prime}\right)\right)\right) r^{\prime 2} \mathrm{~d} r^{\prime} R_{l^{\prime} l}^{m}(\vartheta) \\
& -\int_{0}^{R}\left(\hat{f}_{l}\left(r, r^{\prime}\right) \operatorname{Im}\left(\hat{\phi}_{l^{\prime}}^{m *}(r) \hat{\phi}_{l}^{m}\left(r^{\prime}\right)\right)-\tilde{g}_{l}\left(r, r^{\prime}\right) \operatorname{Im}\left(\psi_{l^{\prime}}^{m *}(r) \psi_{l}^{m}\left(r^{\prime}\right)\right)\right) r^{\prime 2} \mathrm{~d} r^{\prime} \\
& \times \\
& \left.m\left(Q_{l^{\prime} l}^{m}(\vartheta)+Q_{l l^{\prime}}^{m}(\vartheta)\right) / \sin \vartheta\right\} \\
& \\
& \frac{2}{\eta r} \sum_{l, l^{\prime} ; m>0}\left\{-l^{\prime}\left(l^{\prime}+1\right) \int_{0}^{R} \tilde{g}_{l}\left(r, r^{\prime}\right) \operatorname{Re}\left(\phi_{l^{\prime}}^{m *}(r) \psi_{l}^{m}\left(r^{\prime}\right)\right) r^{\prime 2} \mathrm{~d} r^{\prime} Q_{l^{\prime} l}^{m}(\vartheta)\right. \\
& -l(l+1) \int_{0}^{R} f_{l}\left(r, r^{\prime}\right) \operatorname{Re}\left(\psi_{l^{\prime}}^{m *}(r) \hat{\phi}_{l}^{m}\left(r^{\prime}\right)\right) r^{\prime 2} \mathrm{~d} r^{\prime} Q_{l l^{\prime}}^{m}(\vartheta) \\
& +m \int_{0}^{R}\left[l^{\prime}\left(l^{\prime}+1\right) \hat{f}_{l}\left(r, r^{\prime}\right) \operatorname{Im}\left(\phi_{l^{\prime}}^{m *}(r) \hat{\phi}_{l}^{m}\left(r^{\prime}\right)\right)\right. \\
& \left.\left.+l(l+1) f_{l}\left(r, r^{\prime}\right) \operatorname{Im}\left(\hat{\phi}_{l^{\prime}}^{m *}(r) \hat{\phi}_{l}^{m}\left(r^{\prime}\right)\right)\right] r^{\prime 2} \mathrm{~d} r^{\prime} P_{l^{\prime} l}^{m}(\vartheta) / \sin \vartheta\right\}
\end{aligned}
$$




$$
\begin{aligned}
\tilde{a}_{\varphi r}(r, \vartheta)= & \frac{2}{\eta r} \sum_{l, l^{\prime} ; m>0}\left\{l^{\prime}\left(l^{\prime}+1\right) \int_{0}^{R} \hat{f}_{l}\left(r, r^{\prime}\right) \operatorname{Re}\left(\phi_{l^{\prime}}^{m *}(r) \hat{\phi}_{l}^{m}\left(r^{\prime}\right)\right) r^{\prime 2} \mathrm{~d} r^{\prime} Q_{l^{\prime} l}^{m}(\vartheta)\right. \\
& -l(l+1) \int_{0}^{R} f_{l}\left(r, r^{\prime}\right) \operatorname{Re}\left(\hat{\phi}_{l^{\prime}}^{m *}(r) \hat{\phi}_{l}^{m}\left(r^{\prime}\right)\right) r^{\prime 2} \mathrm{~d} r^{\prime} Q_{l l^{\prime}}^{m}(\vartheta) \\
& +m \int_{0}^{R}\left[l^{\prime}\left(l^{\prime}+1\right) \tilde{g}_{l}\left(r, r^{\prime}\right) \operatorname{Im}\left(\phi_{l^{\prime}}^{m *}(r) \psi_{l}^{m}\left(r^{\prime}\right)\right)\right. \\
& \left.\left.-l(l+1) f_{l}\left(r, r^{\prime}\right) \operatorname{Im}\left(\psi_{l^{\prime}}^{m *}(r) \hat{\phi}_{l}^{m}\left(r^{\prime}\right)\right)\right] r^{\prime 2} \mathrm{~d} r^{\prime} P_{l^{\prime} l}^{m}(\vartheta) / \sin \vartheta\right\}
\end{aligned}
$$

$$
\begin{aligned}
\tilde{a}_{r \vartheta}(r, \vartheta)= & \frac{1}{\eta} \sum_{l, l^{\prime}, l^{\prime \prime} ; m>0} \frac{(2 l+1)(l-|m|) !}{l(l+1)(l+|m|) !} \\
& \times\left\{R _ { l ^ { \prime } l } ^ { m } ( \vartheta ) \left[-m \int_{0}^{R}\left\{l(l+1) g_{l}\left(r, r^{\prime}\right) \operatorname{Im}\left(\hat{\phi}_{l^{\prime}}^{m *}(r) \hat{\phi}_{l^{\prime \prime}}^{m}\left(r^{\prime}\right)\right)\right.\right.\right. \\
& \left.+l^{\prime \prime}\left(l^{\prime \prime}+1\right) \tilde{g}_{l}\left(r, r^{\prime}\right) \operatorname{Im}\left(\hat{\phi}_{l^{\prime}}^{m *}(r) \phi_{l^{\prime \prime}}^{m}\left(r^{\prime}\right)\right)\right\} r^{\prime} \mathrm{d} r^{\prime} \int_{0}^{\pi} P_{l^{\prime \prime} l}^{m}\left(\vartheta^{\prime}\right) \mathrm{d} \vartheta^{\prime} \\
& -l(l+1) \int_{0}^{R} g_{l}\left(r, r^{\prime}\right) \operatorname{Re}\left(\hat{\phi}_{l^{\prime}}^{m *}(r) \psi_{l^{\prime \prime}}^{m}\left(r^{\prime}\right)\right) r^{\prime} \mathrm{d} r^{\prime} \int_{0}^{\pi} Q_{l l^{\prime \prime}}^{m}\left(\vartheta^{\prime}\right) \sin \vartheta^{\prime} \mathrm{d} \vartheta^{\prime} \\
& \left.-l^{\prime \prime}\left(l^{\prime \prime}+1\right) \int_{0}^{R} \hat{f_{l}}\left(r, r^{\prime}\right) \operatorname{Re}\left(\psi_{l^{\prime}}^{m *}(r) \phi_{l^{\prime \prime}}^{m}\left(r^{\prime}\right)\right) r^{\prime} \mathrm{d} r^{\prime} \int_{0}^{\pi} Q_{l^{\prime \prime} l}^{m}\left(\vartheta^{\prime}\right) \sin \vartheta^{\prime} \mathrm{d} \vartheta^{\prime}\right] \\
& +m\left(Q_{l^{\prime} l}^{m}(\vartheta)+Q_{l l^{\prime}}^{m}(\vartheta)\right) / \sin \vartheta\left[m \int _ { 0 } ^ { R } \left\{l(l+1) g_{l}\left(r, r^{\prime}\right) \operatorname{Re}\left(\psi_{l^{\prime}}^{m *}(r) \hat{\phi}_{l^{\prime \prime}}^{m}\left(r^{\prime}\right)\right)\right.\right. \\
& \left.+l^{\prime \prime}\left(l^{\prime \prime}+1\right) \tilde{g}_{l}\left(r, r^{\prime}\right) \operatorname{Re}\left(\psi_{l^{\prime}}^{m *}(r) \phi_{l^{\prime \prime}}^{m}\left(r^{\prime}\right)\right)\right\} r^{\prime} \mathrm{d} r^{\prime} \int_{0}^{\pi} P_{l^{\prime \prime} l}^{m}\left(\vartheta^{\prime}\right) \mathrm{d} \vartheta^{\prime} \\
& -l(l+1) \int_{0}^{R} g_{l}\left(r, r^{\prime}\right) \operatorname{Im}\left(\psi_{l^{\prime}}^{m *}(r) \psi_{l^{\prime \prime}}^{m}\left(r^{\prime}\right)\right) r^{\prime} \mathrm{d} r^{\prime} \int_{0}^{\pi} Q_{l l^{\prime \prime}}^{m}\left(\vartheta^{\prime}\right) \sin \vartheta^{\prime} \mathrm{d} \vartheta^{\prime} \\
& +l^{\prime \prime}\left(l^{\prime \prime}+1\right) \int_{0}^{R} \hat{f}_{l}\left(r, r^{\prime}\right) \operatorname{Im}\left(\hat{\phi}_{l^{\prime}}^{m *}(r) \phi_{l^{\prime \prime}}^{m}\left(r^{\prime}\right)\right) r^{\prime} \mathrm{d} r^{\prime} \\
& \left.\times \int_{0}^{\pi} Q_{l^{\prime \prime} l}^{m}\left(\vartheta^{\prime}\right) \sin \vartheta^{\prime} \mathrm{d} \vartheta^{\prime}\right\}
\end{aligned}
$$




$$
\begin{aligned}
\tilde{a}_{\vartheta \vartheta}(r, \vartheta)= & \frac{1}{\eta r} \sum_{l, l^{\prime}, l^{\prime \prime} ; m>0} \frac{(2 l+1)(l-|m|) !}{l(l+1)(l+|m|) !} \\
& \times\left\{l ^ { \prime } ( l ^ { \prime } + 1 ) Q _ { l ^ { \prime } l } ^ { m } ( \vartheta ) \left[m \int _ { 0 } ^ { R } \left\{l(l+1) g_{l}\left(r, r^{\prime}\right) \operatorname{Im}\left(\phi_{l^{\prime}}^{m *}(r) \hat{\phi}_{l^{\prime \prime}}^{m}\left(r^{\prime}\right)\right)\right.\right.\right. \\
& \left.+l^{\prime \prime}\left(l^{\prime \prime}+1\right) \tilde{g}_{l}\left(r, r^{\prime}\right) \operatorname{Im}\left(\phi_{l^{\prime}}^{m *}(r) \phi_{l^{\prime \prime}}^{m}\left(r^{\prime}\right)\right)\right\} r^{\prime} \mathrm{d} r^{\prime} \int_{0}^{\pi} P_{l^{\prime \prime} l}^{m}\left(\vartheta^{\prime}\right) \mathrm{d} \vartheta^{\prime} \\
& \left.+l(l+1) \int_{0}^{R} g_{l}\left(r, r^{\prime}\right) \operatorname{Re}\left(\phi_{l^{\prime}}^{m *}(r) \psi_{l^{\prime \prime}}^{m}\left(r^{\prime}\right)\right) r^{\prime} \mathrm{d} r^{\prime} \int_{0}^{\pi} Q_{l l^{\prime \prime}}^{m}\left(\vartheta^{\prime}\right) \sin \vartheta^{\prime} \mathrm{d} \vartheta^{\prime}\right] \\
& -l^{\prime \prime}\left(l^{\prime \prime}+1\right) \int_{0}^{\pi} Q_{l^{\prime \prime} l}^{m}\left(\vartheta^{\prime}\right) \sin \vartheta^{\prime} \mathrm{d} \vartheta^{\prime}\left[m \int _ { 0 } ^ { R } \left\{l(l+1) f_{l}\left(r, r^{\prime}\right) \operatorname{Im}\left(\hat{\phi}_{l^{\prime}}^{m *}(r) \phi_{l^{\prime \prime}}^{m}\left(r^{\prime}\right)\right)\right.\right. \\
& \left.+l^{\prime}\left(l^{\prime}+1\right) \hat{f}_{l}\left(r, r^{\prime}\right) \operatorname{Im}\left(\phi_{l^{\prime}}^{m *}(r) \phi_{l^{\prime \prime}}^{m}\left(r^{\prime}\right)\right)\right\} r^{\prime} \mathrm{d} r^{\prime} P_{l^{\prime} l}^{m}(\vartheta) / \sin \vartheta \\
& \left.\left.-l(l+1) \int_{0}^{R} f_{l}\left(r, r^{\prime}\right) \operatorname{Re}\left(\psi_{l^{\prime}}^{m *}(r) \phi_{l^{\prime \prime}}^{m}\left(r^{\prime}\right)\right) r^{\prime} \mathrm{d} r^{\prime} Q_{l l^{\prime}}^{m}(\vartheta)\right]\right\}
\end{aligned}
$$

$$
\begin{aligned}
\tilde{a}_{\varphi \vartheta}(r, \vartheta)= & \frac{1}{\eta r} \sum_{l, l^{\prime}, l^{\prime \prime} ; m>0} \frac{(2 l+1)(l-|m|) !}{l(l+1)(l+|m|) !} \\
& \times\left\{m l^{\prime}\left(l^{\prime}+1\right) P_{l^{\prime} l}^{m}(\vartheta) / \sin \vartheta\left[m \int _ { 0 } ^ { R } \left\{l(l+1) g_{l}\left(r, r^{\prime}\right) \operatorname{Re}\left(\phi_{l^{\prime}}^{m *}(r) \hat{\phi}_{l^{\prime \prime}}^{m}\left(r^{\prime}\right)\right)\right.\right.\right. \\
& \left.+l^{\prime \prime}\left(l^{\prime \prime}+1\right) \tilde{g}_{l}\left(r, r^{\prime}\right) \operatorname{Re}\left(\phi_{l^{\prime}}^{m *}(r) \phi_{l^{\prime \prime}}^{m}\left(r^{\prime}\right)\right)\right\} r^{\prime} \mathrm{d} r^{\prime} \int_{0}^{\pi} P_{l^{\prime \prime} l}^{m}\left(\vartheta^{\prime}\right) \mathrm{d} \vartheta^{\prime} \\
& \left.-l(l+1) \int_{0}^{R} g_{l}\left(r, r^{\prime}\right) \operatorname{Im}\left(\phi_{l^{\prime}}^{m *}(r) \psi_{l^{\prime \prime}}^{m}\left(r^{\prime}\right)\right) r^{\prime} \mathrm{d} r^{\prime} \int_{0}^{\pi} Q_{l l^{\prime \prime}}^{m}\left(\vartheta^{\prime}\right) \sin \vartheta^{\prime} \mathrm{d} \vartheta^{\prime}\right] \\
& +l^{\prime \prime}\left(l^{\prime \prime}+1\right) \int_{0}^{\pi} Q_{l^{\prime \prime} l}^{m}\left(\vartheta^{\prime}\right) \sin \vartheta^{\prime} \mathrm{d} \vartheta^{\prime} \\
& {\left[m l(l+1) P_{l^{\prime} l}^{m}(\vartheta) / \sin \vartheta \int_{0}^{R} f_{l}\left(r, r^{\prime}\right) \operatorname{Im}\left(\psi_{l^{\prime}}^{m *}(r) \phi_{l^{\prime \prime}}^{m}\left(r^{\prime}\right)\right) r^{\prime} \mathrm{d} r^{\prime}\right.} \\
& l^{\prime}\left(l^{\prime}+1\right) Q_{l^{\prime} l}^{m}(\vartheta) \int_{0}^{R} \hat{f}_{l}\left(r, r^{\prime}\right) \operatorname{Re}\left(\phi_{l^{\prime}}^{m *}(r) \phi_{l^{\prime \prime}}^{m}\left(r^{\prime}\right)\right) r^{\prime} \mathrm{d} r^{\prime} \\
& \left.\left.+l(l+1) Q_{l l^{\prime}}^{m}(\vartheta) \int_{0}^{R} f_{l}\left(r, r^{\prime}\right) \operatorname{Re}\left(\hat{\phi}_{l^{\prime}}^{m *}(r) \phi_{l^{\prime \prime}}^{m}\left(r^{\prime}\right)\right) r^{\prime} \mathrm{d} r^{\prime}\right]\right\}
\end{aligned}
$$




$$
\begin{aligned}
\tilde{a}_{\vartheta \varphi}(r, \vartheta)= & \frac{1}{\eta r} \sum_{l, l^{\prime}, l^{\prime \prime} ; m>0} \frac{(2 l+1)(l-|m|) !}{l(l+1)(l+|m|) !} \\
& \times\left\{l ^ { \prime } ( l ^ { \prime } + 1 ) Q _ { l ^ { \prime } l } ^ { m } ( \vartheta ) \left[-m l(l+1) \int_{0}^{R} g_{l}\left(r, r^{\prime}\right) \operatorname{Im}\left(\phi_{l^{\prime}}^{m *}(r) \psi_{l^{\prime \prime}}^{m}\left(r^{\prime}\right)\right) r^{\prime} \mathrm{d} r^{\prime} \int_{0}^{\pi} P_{l l^{\prime \prime}}^{m}\left(\vartheta^{\prime}\right) \mathrm{d} \vartheta^{\prime}\right.\right. \\
& -l^{\prime \prime}\left(l^{\prime \prime}+1\right) \int_{0}^{R} \tilde{g}_{l}\left(r, r^{\prime}\right) \operatorname{Re}\left(\phi_{l^{\prime}}^{m *}(r) \phi_{l^{\prime \prime}}^{m}\left(r^{\prime}\right)\right) r^{\prime} \mathrm{d} r^{\prime} \int_{0}^{\pi} Q_{l^{\prime \prime} l}^{m}\left(\vartheta^{\prime}\right) \sin \vartheta^{\prime} \mathrm{d} \vartheta^{\prime} \\
& \left.+l(l+1) \int_{0}^{R} g_{l}\left(r, r^{\prime}\right) \operatorname{Re}\left(\phi_{l^{\prime}}^{m *}(r) \hat{\phi}_{l^{\prime \prime}}^{m}\left(r^{\prime}\right)\right) r^{\prime} \mathrm{d} r^{\prime} \int_{0}^{\pi} Q_{l l^{\prime \prime}}^{m}\left(\vartheta^{\prime}\right) \sin \vartheta^{\prime} \mathrm{d} \vartheta^{\prime}\right] \\
& +m l^{\prime \prime}\left(l^{\prime \prime}+1\right) \int_{0}^{\pi} P_{l^{\prime \prime} l}^{m}\left(\vartheta^{\prime}\right) \mathrm{d} \vartheta^{\prime}\left[m \int _ { 0 } ^ { R } \left\{l(l+1) f_{l}\left(r, r^{\prime}\right) \operatorname{Re}\left(\hat{\phi}_{l^{\prime}}^{m *}(r) \phi_{l^{\prime \prime}}^{m}\left(r^{\prime}\right)\right)\right.\right. \\
& \left.+l^{\prime}\left(l^{\prime}+1\right) \hat{f}_{l}\left(r, r^{\prime}\right) \operatorname{Re}\left(\phi_{l^{\prime}}^{m *}(r) \phi_{l^{\prime \prime}}^{m}\left(r^{\prime}\right)\right)\right\} r^{\prime} \mathrm{d} r^{\prime} P_{l^{\prime} l}^{m}(\vartheta) / \sin \vartheta \\
& \left.\left.+l(l+1) \int_{0}^{R} f_{l}\left(r, r^{\prime}\right) \operatorname{Im}\left(\psi_{l^{\prime}}^{m *}(r) \phi_{l^{\prime \prime}}^{m}\left(r^{\prime}\right)\right) r^{\prime} \mathrm{d} r^{\prime} Q_{l l^{\prime}}^{m}(\vartheta)\right]\right\}
\end{aligned}
$$

$$
\begin{aligned}
\tilde{a}_{r \varphi}(r, \vartheta)= & -\frac{1}{\eta} \sum_{l, l^{\prime}, l^{\prime \prime} ; m>0} \frac{(2 l+1)(l-|m|) !}{l(l+1)(l+|m|) !} \\
& \times\left\{R _ { l ^ { \prime } l } ^ { m } ( \vartheta ) \left[-m \int_{0}^{R}\left\{l(l+1) g_{l}\left(r, r^{\prime}\right) \operatorname{Im}\left(\hat{\phi}_{l^{\prime}}^{m *}(r) \psi_{l^{\prime \prime}}^{m}\left(r^{\prime}\right)\right)\right.\right.\right. \\
& \left.-l^{\prime \prime}\left(l^{\prime \prime}+1\right) \hat{f}_{l}\left(r, r^{\prime}\right) \operatorname{Im}\left(\psi_{l^{\prime}}^{m *}(r) \phi_{l^{\prime \prime}}^{m}\left(r^{\prime}\right)\right)\right\} r^{\prime} \mathrm{d} r^{\prime} \int_{0}^{\pi} P_{l^{\prime \prime} l}^{m}\left(\vartheta^{\prime}\right) \mathrm{d} \vartheta^{\prime} \\
& +l(l+1) \int_{0}^{R} g_{l}\left(r, r^{\prime}\right) \operatorname{Re}\left(\hat{\phi}_{l^{\prime}}^{m *}(r) \hat{\phi}_{l^{\prime \prime}}^{m}\left(r^{\prime}\right)\right) r^{\prime} \mathrm{d} r^{\prime} \int_{0}^{\pi} Q_{l l^{\prime \prime}}^{m}\left(\vartheta^{\prime}\right) \sin \vartheta^{\prime} \mathrm{d} \vartheta^{\prime} \\
& \left.-l^{\prime \prime}\left(l^{\prime \prime}+1\right) \int_{0}^{R} \tilde{g}_{l}\left(r, r^{\prime}\right) \operatorname{Re}\left(\hat{\phi}_{l^{\prime}}^{m *}(r) \phi_{l^{\prime \prime}}^{m}\left(r^{\prime}\right)\right) r^{\prime} \mathrm{d} r^{\prime} \int_{0}^{\pi} Q_{l^{\prime \prime} l}^{m}\left(\vartheta^{\prime}\right) \sin \vartheta^{\prime} \mathrm{d} \vartheta^{\prime}\right] \\
& +m\left(Q_{l^{\prime} l}^{m}(\vartheta)+Q_{l l^{\prime}}^{m}(\vartheta)\right) / \sin \vartheta\left[m \int _ { 0 } ^ { R } \left\{l(l+1) g_{l}\left(r, r^{\prime}\right) \operatorname{Re}\left(\psi_{l^{\prime}}^{m *}(r) \psi_{l^{\prime \prime}}^{m}\left(r^{\prime}\right)\right)\right.\right. \\
& \left.+l^{\prime \prime}\left(l^{\prime \prime}+1\right) \hat{f}_{l}\left(r, r^{\prime}\right) \operatorname{Re}\left(\hat{\phi}_{l^{\prime}}^{m *}(r) \phi_{l^{\prime \prime}}^{m}\left(r^{\prime}\right)\right)\right\} r^{\prime} \mathrm{d} r^{\prime} \int_{0}^{\pi} P_{l^{\prime \prime} l}^{m}\left(\vartheta^{\prime}\right) \mathrm{d} \vartheta^{\prime} \\
& +l(l+1) \int_{0}^{R} g_{l}\left(r, r^{\prime}\right) \operatorname{Im}\left(\psi_{l^{\prime}}^{m *}(r) \hat{\phi}_{l^{\prime \prime}}^{m}\left(r^{\prime}\right)\right) r^{\prime} \mathrm{d} r^{\prime} \int_{0}^{\pi} Q_{l l^{\prime \prime}}^{m}\left(\vartheta^{\prime}\right) \sin \vartheta^{\prime} \mathrm{d} \vartheta^{\prime} \\
& -l^{\prime \prime}\left(l^{\prime \prime}+1\right) \int_{0}^{R} \tilde{g}_{l}\left(r, r^{\prime}\right) \operatorname{Im}\left(\psi_{l^{\prime}}^{m *}(r) \phi_{l^{\prime \prime}}^{m}\left(r^{\prime}\right)\right) r^{\prime} \mathrm{d} r^{\prime} \\
& \left.\times \int_{0}^{\pi} Q_{l^{\prime \prime} l}^{m}\left(\vartheta^{\prime}\right) \sin \vartheta^{\prime} \mathrm{d} \vartheta^{\prime}\right\}
\end{aligned}
$$




$$
\begin{aligned}
\tilde{a}_{\varphi \varphi}(r, \vartheta)= & -\frac{1}{\eta r} \sum_{l, l^{\prime}, l^{\prime \prime} ; m>0} \frac{(2 l+1)(l-|m|) !}{l(l+1)(l+|m|) !} \\
& \times\left\{m l^{\prime}\left(l^{\prime}+1\right) P_{l^{\prime} l}^{m}(\vartheta) / \sin \vartheta\right. \\
& \times\left[m l(l+1) \int_{0}^{R} g_{l}\left(r, r^{\prime}\right) \operatorname{Re}\left(\phi_{l^{\prime}}^{m *}(r) \psi_{l^{\prime \prime}}^{m}\left(r^{\prime}\right)\right) r^{\prime} \mathrm{d} r^{\prime} \int_{0}^{\pi} P_{l l^{\prime \prime}}^{m}\left(\vartheta^{\prime}\right) \mathrm{d} \vartheta^{\prime}\right. \\
& -l^{\prime \prime}\left(l^{\prime \prime}+1\right) \int_{0}^{R} \tilde{g}_{l}\left(r, r^{\prime}\right) \operatorname{Im}\left(\phi_{l^{\prime}}^{m *}(r) \phi_{l^{\prime \prime}}^{m}\left(r^{\prime}\right)\right) r^{\prime} \mathrm{d} r^{\prime} \int_{0}^{\pi} Q_{l^{\prime \prime} l}^{m}\left(\vartheta^{\prime}\right) \sin \vartheta^{\prime} \mathrm{d} \vartheta^{\prime} \\
& \left.+l(l+1) \int_{0}^{R} g_{l}\left(r, r^{\prime}\right) \operatorname{Im}\left(\phi_{l^{\prime}}^{m *}(r) \hat{\phi}_{l^{\prime \prime}}^{m}\left(r^{\prime}\right)\right) r^{\prime} \mathrm{d} r^{\prime} \int_{0}^{\pi} Q_{l l^{\prime \prime}}^{m}\left(\vartheta^{\prime}\right) \sin \vartheta^{\prime} \mathrm{d} \vartheta^{\prime}\right] \\
& +m l^{\prime \prime}\left(l^{\prime \prime}+1\right) \int_{0}^{\pi} P_{l^{\prime \prime} l}^{m}\left(\vartheta^{\prime}\right) \mathrm{d} \vartheta^{\prime} \\
& \times\left[m l(l+1) P_{l^{\prime} l}^{m}(\vartheta) / \sin \vartheta \int_{0}^{R} f_{l}\left(r, r^{\prime}\right) \operatorname{Re}\left(\psi_{l^{\prime}}^{m *}(r) \phi_{l^{\prime \prime}}^{m}\left(r^{\prime}\right)\right) r^{\prime} \mathrm{d} r^{\prime}\right. \\
& +l^{\prime}\left(l^{\prime}+1\right) Q_{l^{\prime} l}^{m}(\vartheta) \int_{0}^{R} \hat{f}_{l}\left(r, r^{\prime}\right) \operatorname{Im}\left(\phi_{l^{\prime}}^{m *}(r) \phi_{l^{\prime \prime}}^{m}\left(r^{\prime}\right)\right) r^{\prime} \mathrm{d} r^{\prime} \\
& \left.\left.-l(l+1) Q_{l l^{\prime}}^{m}(\vartheta) \int_{0}^{R} f_{l}\left(r, r^{\prime}\right) \operatorname{Im}\left(\hat{\phi}_{l^{\prime}}^{m *}(r) \phi_{l^{\prime \prime}}^{m}\left(r^{\prime}\right)\right) r^{\prime} \mathrm{d} r^{\prime}\right]\right\}
\end{aligned}
$$





\section{E Comparison between approach (I) and approach (II)}
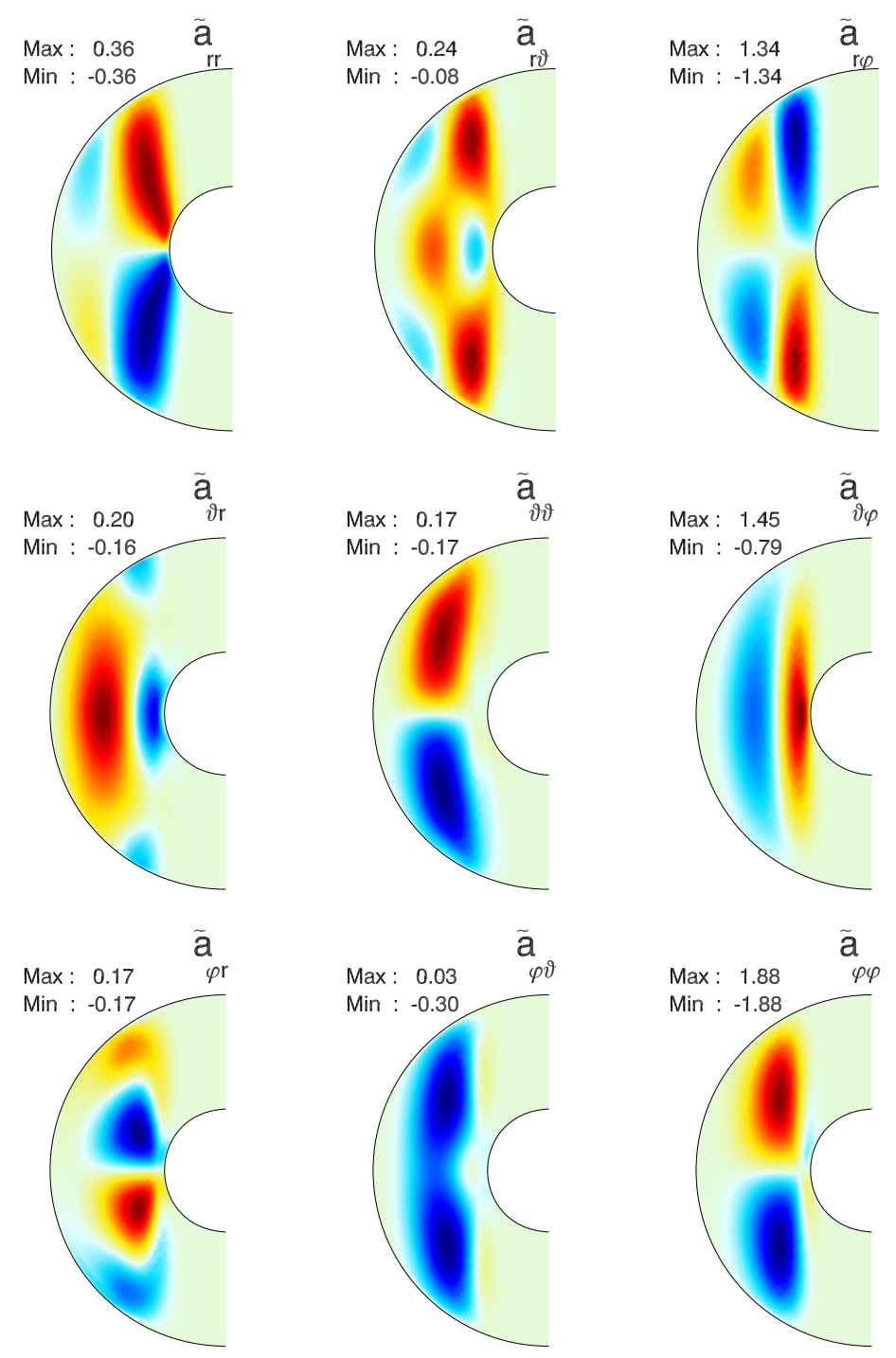

Figure E.1: Mean-field coefficients determined by method (I) for the example of magnetoconvection, but with $P m=0.1$. Maxima and minima are given in units of $\nu / D$. 

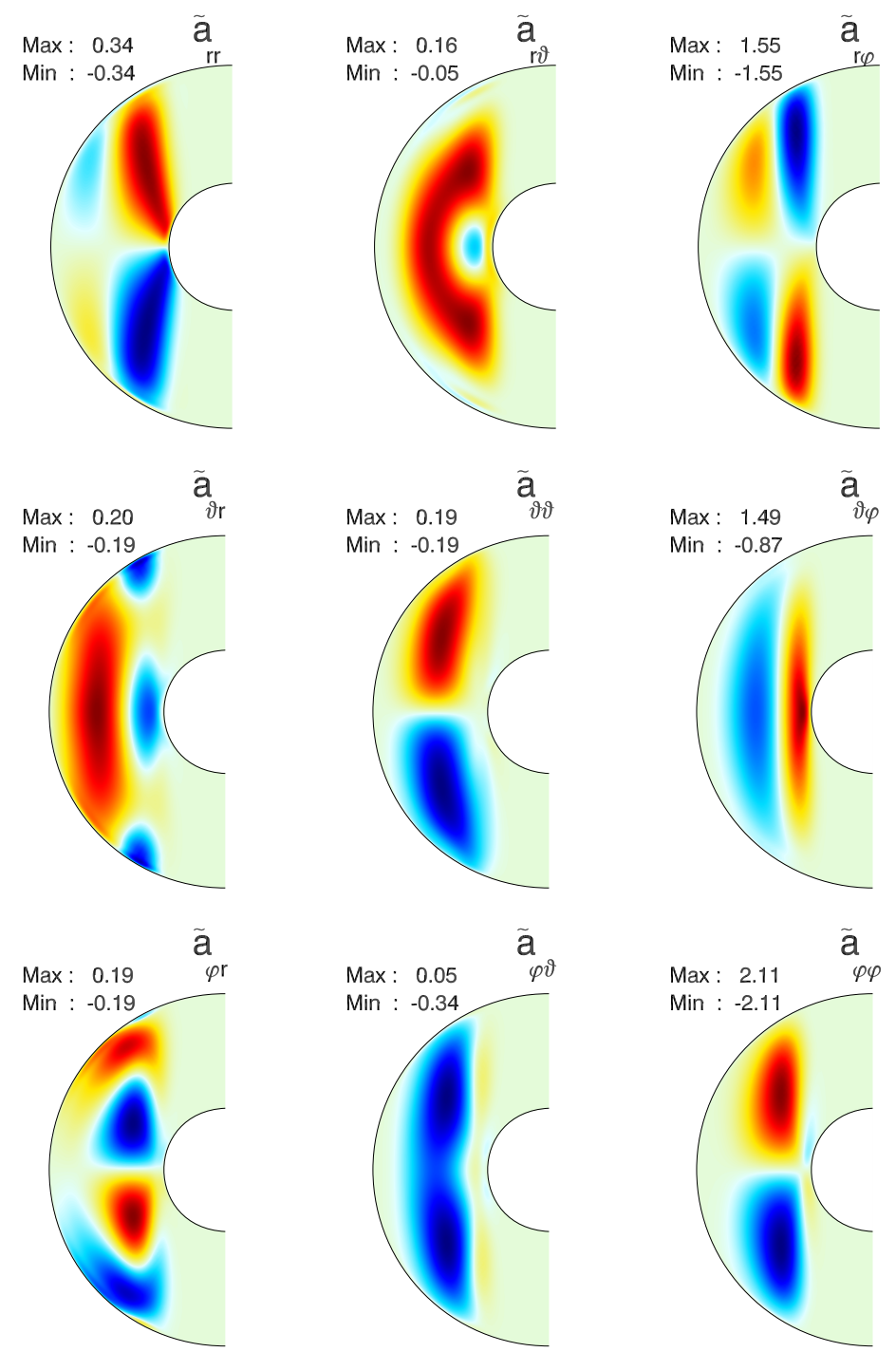

Figure E.2: Mean-field coefficients determined by method (II), otherwise corresponding to those shown in Figure E.1.

Mean-field coefficients determined by approach (I) and approach (II) are displayed in Figures E.1 and E.2. Since approach (II) is based on the second order correlation approximation, $R m$ has been scaled down until both sets of coefficients have come into reasonable agreement. Slight differences which still exist might originate from numerical errors which occur carrying out the numerical integrations over $r^{\prime}$ and $\vartheta^{\prime}$ in (4.38) and (D.1-D.9) for a limited spatial resolution. Standard library routines based on finite difference formulas have been used for this purpose. The neglect of the mean flow for the analytical determination of $\tilde{a}_{\kappa \lambda}$ has no noticeable effect at low magnetic Reynolds numbers. 


\section{F Mean-field coefficients for the example of the benchmark dynamo}
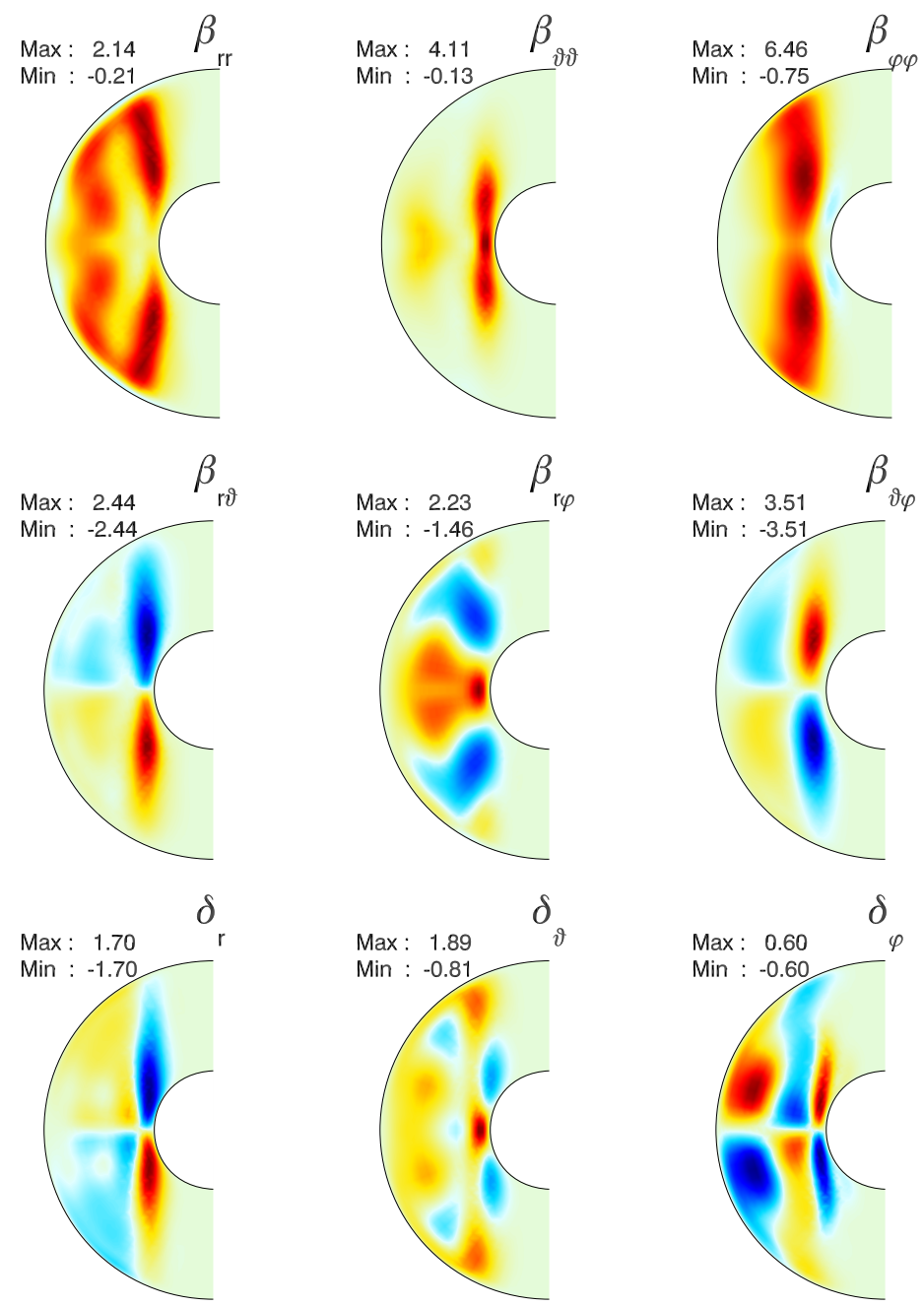

Figure F.1: $\beta$ - and $\delta$-components in the example of the benchmark dynamo. Maxima and minima are given in units of $\nu$. Carrying out the mean-field calculations, the small negative patches in $\beta_{\varphi \varphi}$ have been neglected for numerical reasons. Note that this has no noticeable effect on the mean electromotive force. 

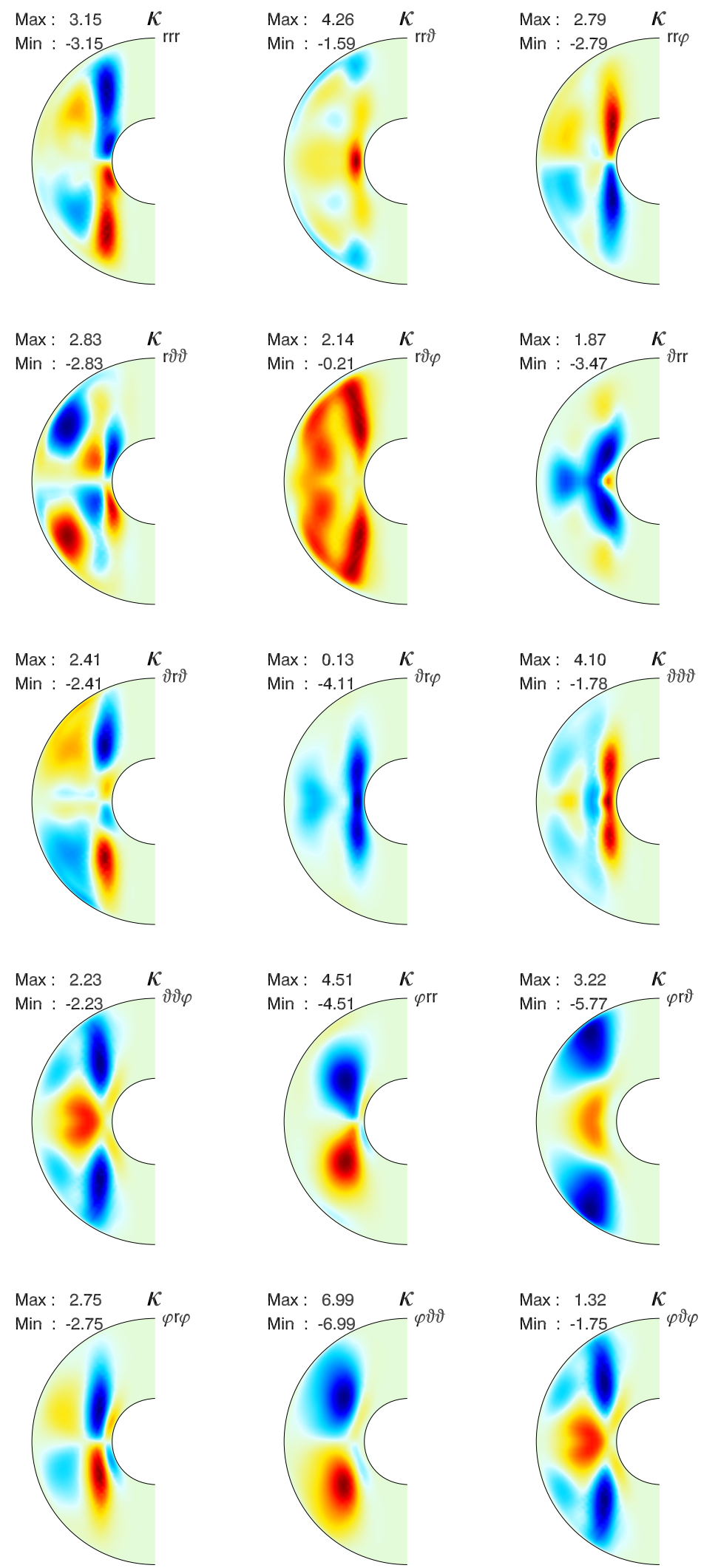

Figure F.2: $\kappa$-components in the example of the benchmark dynamo. Maxima and minima are given in units of $\nu$. 


\section{Bibliography}

Abramovich, M., Stegun, I. A., 1965, Handbook of Mathematical Functions, Dover

Beck, R., Brandenburg, A., Moss, D., Shukurov, A., Sokoloff, D., 1996, Galactic Magnetism: Recent Developments and Perspectives, Annual Review of Astronomy \& Astrophysics, 34, 155-206

Brandenburg, A., Sokoloff, D., 2002, Local and nonlocal magnetic diffusion and alphaeffect tensors in shear flow turbulence, Geophysical \& Astrophysical Fluid Dynamics, 96, 319-344

Brandenburg, A., Nordlund, A., Stein, R. F., Torkelsson, U., 1995, Dynamo-generated Turbulence and Large-Scale Magnetic Fields in a Keplerian Shear Flow, Astrophysical Journal, 446, 741-754

Brandenburg, A., Jennings, R. L., Nordlund, Å., Rieutord, M., Stein, R. F., Tuominen, I., 1996, Magnetic structures in a dynamo simulation, Journal of Fluid Mechanics, 306, 325-352

Brun, A. S., Miesch, M. S., Toomre, J., 2004, Global-Scale Turbulent Convection and Magnetic Dynamo Action in the Solar Envelope, Astrophysical Journal, 614, 10731098

Cattaneo, F., Hughes, D. W., 1996, Nonlinear saturation of the turbulent $\alpha$ effect, Physical Review E, 54, 4532-4535

Christensen, U., Olson, P., Glatzmaier, G. A., 1998, A dynamo model interpretation of geomagnetic field structures, Geophysical Research Letters, 25, 1565-1568

Christensen, U., Olson, P., Glatzmaier, G. A., 1999, Numerical modelling of the geodynamo: a systematic parameter study, Geophysical Journal International, 138, 393-409

Christensen, U. R., Aubert, J., Cardin, P., Dormy, E., Gibbons, S., Glatzmaier, G. A., Grote, E., Honkura, Y., Jones, C., Kono, M., Matsushima, M., Sakuraba, A., Takahashi, F., Tilgner, A., Wicht, J., Zhang, K., 2001, A numerical dynamo benchmark, Physics of the Earth and Planetary Interiors, 128, 25-34

Cowling, T., 1933, Magnetic field of sunspots, Monthly Notices of the Royal Astronomical Society, 94, 39-48

Fearn, D., 1998, Hydromagnetic flow in planetary cores, Reports on Progress in Physics, $61,175-235$ 
Ferrière, K., 1992, Effect of an ensamble of explosions on the galactic dynamo, Astrophysical Journal, 389, 286-296

Ferrière, K., 1993a, The full alpha-tensor due to supernova and superbubbles in the galactic disk, Astrophysical Journal, 404, 162-184

Ferrière, K., 1993b, Magnetic diffusion due to supernova explosions and superbubbles in the galactic disk, Astrophysical Journal, 409, 248-261

Feudel, F., Gellert, M., Rüdiger, S., Witt, A., Seehafer, N., 2003, Dynamo effect in a driven helical flow, Physical Review E, 68, 046302

Gailitis, A., Lielausis, O., Dement'ev, S., Platacis, E., Cifersons, A., Gerbeth, G., Gundrum, T., Stefani, F., Christen, M., Hänel, H., Will, G., 2000, Detection of a Flow Induced Magnetic Field Eigenmode in the Riga Dynamo Facility, Physical Review Letters, 84, 4365-4368

Gilman, P. A., 1983, Dynamically consistent nonlinear dynamos driven by convection in a rotating spherical shell. II - Dynamos with cycles and strong feedbacks, Astrophysical Journal Supplement Series, 53, 243-268

Gilman, P. A., Miller, J., 1981, Dynamically consistent nonlinear dynamos driven by convection in a rotating spherical shell, Astrophysical Journal Supplement Series, 46, 211-238

Glatzmaier, G. A., 1984, Numerical simulations of stellar convective dynamos. I - The model and method, Journal of Computational Physics, 55, 461-484

Glatzmaier, G. A., 1985, Numerical simulations of stellar convective dynamos. II - Field propagation in the convection zone, Astrophysical Journal, 291, 300-307

Glatzmaier, G. A., Roberts, P. H., 1995a, A three-dimensional self-consistent computer simulation of a geomagnetic field reversal, Nature, 377, 203-208

Glatzmaier, G. A., Roberts, P. H., 1995b, A three-dimensional convective dynamo solution with rotating and finitely conducting inner core and mantle, Physics of the Earth and Planetary Interiors, 91, 63-75

Gruzinov, A. V., Diamond, P. H., 1994, Self-consistent theory of mean-field electrodynamics, Physical Review Letters, 72, 1651-1653

Gruzinov, A. V., Diamond, P. H., 1995, Self-consistent mean field electrodynamics of turbulent dynamos, Physics of Plasmas, 2, 1941-1946

Gruzinov, A. V., Diamond, P. H., 1996, Nonlinear mean field electrodynamics of turbulent dynamos, Physics of Plasmas, 3, 1853-1857

Gubbins, D., 1974, Theories of the Geomagnetic and Solar Dynamos, Reviews of Geophysics and Space Physics, 12, 137-154 
Gubbins, D., Zhang, K., 1993, Symmetry properties of the dynamo equations for palaeomagnetism and geomagnetism, Physics of the Earth and Planetary Interiors, 75, 225241

Hawley, J. F., Gammie, C. F., Balbus, S. A., 1996, Local Three-dimensional Simulations of an Accretion Disk Hydromagnetic Dynamo, Astrophysical Journal, 464, 690-703

Hoyng, P., Ossendrijver, M. A. J. H., Schmitt, D., 2001, The geodynamo as a bistable oscillator, Geophysical and Astrophysical Fluid Dynamics, 94, 263-314

Kageyama, A., Sato, T., 1997, Generation mechanism of a dipole field by a magnetohydrodynamic dynamo, Physical Review E, 55, 4617-4626

Kichatinov, L. L., 1991, Turbulent transport of magnetic fields in a highly conducting rotating fluid and the solar cycle, Astronomy \& Astrophysics, 243, 483-491

Kichatinov, L. L., Rüdiger, G., 1992, Magnetic-field advection in inhomogeneous turbulence, Astronomy \& Astrophysics, 260, 494-498

Kleeorin, N., Rogachevskii, I., 2003, Effect of rotation on a developed turbulent stratified convection: The hydrodynamic helicity, the $\alpha$ effect, and the effective drift velocity, Physical Review E, 67, 026321

Krause, F., Rädler, K.-H., 1980, Mean-Field Magnetohydrodynamics and Dynamo Theory, Pergamon Press

Kuang, W., Bloxham, J., 1997, An Earth-like numerical dynamo model, Nature, 389, $371-374$

Kutzner, C., Christensen, U. R., 2002, From stable dipolar towards reversing numerical dynamos, Physics of the Earth and Planetary Interiors, 131, 29-45

Larmor, J., 1919, How could a rotating body such as the sun become a magnet?, British Association for the Advancement of Sciences Report, pp. 159-160

Müller, U., Stieglitz, R., 2000, Experimental Demonstration of a Homogeneous TwoScale Dynamo, APS Meeting Abstracts

McKee, S., Wall, D. P., Wilson, S., 1996, An alternating direction implicit scheme for parabolic equations with mixed derivative and convective terms, Journal of Computational Physics, 126, 64-76

Merrill, R. T., McElhinny, M. W., McFadden, P. L., 1998, The magnetic field of the Earth, Academic Press

Moffatt, H., 1978, Magnetic field generation in electrically conducting fluids, Cambridge University Press

Olson, P., Glatzmaier, G. A., 1995, Magnetoconvection in a rotating spherical shell: structure of flow in the outer core, Physics of the Earth and Planetary Interiors, 92, 109-118 
Olson, P., Christensen, U., Glatzmaier, G. A., 1999, Numerical modeling of the geodynamo: Mechanisms of field generation and equilibration, Journal of Geophysical Research, 104, 10 383-10404

Orszag, S. A., 1970, Analytical theories of turbulence, Journal of Fluid Mechanics, 41, 363-386

Ossendrijver, M., 2003, The solar dynamo, The Astronomy \& Astrophysics Review, 11, 287-367

Ossendrijver, M., Stix, M., Brandenburg, A., 2001, Magnetoconvection and dynamo coefficients:. Dependence of the alpha effect on rotation and magnetic field, Astronomy \& Astrophysics, 376, 713-726

Ossendrijver, M., Stix, M., Brandenburg, A., Rüdiger, G., 2002, Magnetoconvection and dynamo coefficients, Astronomy \& Astrophysics, 394, 735-745

Parker, E. N., 1955, Hydromagnetic Dynamo Models., Astrophysical Journal, 122, 293

Parker, E. N., 1957, The Solar Hydromagnetic Dynamo, Proceedings of the National Academy of Science, pp. 8-14

Pouquet, A., Frisch, U., Léorat, J., 1976, Strong MHD helical turbulence and the nonlinear dynamo effect, Journal of Fluid Mechanics, 77, 321-354

Rädler, K. H., 1969a, On the Electrodynamics of Turbulent Fields under the Influence of Corilois Forces, Monatsbericht der Deutschen Akademie der Wissenschaften, Berlin, Volume 11, p. 194-201, 11, 194-201

Rädler, K. H., 1969b, A New Turbulent Dynamo. I., Monatsbericht der Deutschen Akademie der Wissenschaften, Berlin, Volume 11, p. 272-279, 11, 272-279

Rädler, K.-H., Rheinhardt, M., Apstein, E., Fuchs, H., 2002, On the mean-field theory of the Karlsruhe Dynamo Experiment, Nonlinear Processes in Geophysics, 9, 171-187

Rädler, K.-H., 1980, Mean-Field Approach to Spherical Dynamo Models, Astronomische Nachrichten, 301, 101-127

Rädler, K.-H., 1995, Cosmic Dynamos, Reviews of Modern Astronomy, 8, 295-322

Roberts, G. O., 1972, Dynamo Action of Fluid Motions with Two-Dimensional Periodicity, Royal Society of London Philosophical Transactions Series A, 271, 411-454

Roberts, P., Glatzmaier, G., 2000, Geodynamo theory and simulations, Reviews of Modern Physics, 72, 1081-1123

Rogachevskii, I., Kleeorin, N., 2000, Electromotive force for an anisotropic turbulence: Intermediate nonlinearity, Physical Review E, 61, 5202-5210

Rogachevskii, I., Kleeorin, N., 2003, Electromotive force and large-scale magnetic dynamo in a turbulent flow with a mean shear, Physical Review E, 68, 036301 
Rogachevskii, I., Kleeorin, N., 2004, Nonlinear theory of a "shear-current" effect and mean-field magnetic dynamos, Physical Review E, 70, 046310

Rüdiger, G., 1973, Behandlung eines Einfachen Hydromagnetischen Dynamos mittels Lineariserung, Astronomische Nachrichten, 294, 183-186

Rüdiger, G., Kichatinov, L. L., 1993, Alpha-effect and alpha-quenching, Astronomy \& Astrophysics, 269, 581-588

Shukurov, A., 2002, On the origin of galactic magnetic fields, Astrophysics and Space Science, 281, 285-288

Steenbeck, M., Krause, F., 1969, On the Dynamo Theory of Stellar and Planetary Magnetic Fields. I. AC Dynamos of Solar Type, Astronomische Nachrichten, 291, 49-84

Steenbeck, M., Krause, F., Rädler, K. H., 1966, A Calculation of the Mean Electromotive Force in an Electrically Conducting Fluid in Turbulent Motion Under the Influence of Coriolis Forces, Zeitschrift Naturforschung Teil A, 21, 369-376

Stix, M., 1976, Differential rotation and the solar dynamo, Astronomy \& Astrophysics, $47,243-254$

Stix, M., 2002, The Sun, Springer

Tilgner, A., 1997, A kinematic dynamo with a small scale velocity field, Physics Letters A, 226, 75-79

Tilgner, A., 2004, Small Scale Kinematic Dynamos: Beyond the $\alpha$-Effect, Geophysical and Astrophysical Fluid Dynamics, 98, 225-234

Tobias, S. M., 2002, The solar dynamo, Royal Society of London Philosophical Transactions Series A, 360, 2741-2756

Vainshtein, S. I., Cattaneo, F., 1992, Nonlinear restrictions on dynamo action, Astrophysical Journal, 393, 165-171

Weiss, N., 2002, Dynamos in planets, stars and galaxies, Astrophysics \& Geophysics, 43, $3.9-3.14$

Weiss, N. O., Tobias, S. M., 2000, Physical Causes of Solar Activity, Space Science Reviews, 94, 99-112

Ziegler, U., Yorke, H., Kaisig, M., 1996, The role of supernovae for the galactic dynamo, Astronomy \& Astrophysics, 305, 114-124 



\section{Scientific contributions}

Schrinner, M., Deinzer, W., 2001, On the post main sequence expansion of stars with contracting helium cores, Astronomy \& Astrophysics, 379, 496-500

Schrinner, M., Rädler, K.-H., Schmitt, D., Rheinhardt, M., Christensen, U., 2005, Meanfield view on rotating magnetoconvection and a geodynamo model, Astronomische Nachrichten, $326,245-249$

\section{Danksagung}

Diese Arbeit wurde am Max-Planck-Institut für Sonnensystemforschung in KatlenburgLindau angefertigt. Ich danke stellvertretend der Institutsleitung für die Bereitstellung eines Arbeitsplatzes und die Gewährung eines Stipendiums.

Mein besonderer Dank gilt Dr. D. Schmitt, der mich anfänglich für diese Arbeit begeisterte und in den folgenden drei Jahren unermüdlich unterstützt hat. Sehr viel verdanke ich Prof. K.-H. Rädler sowie Dr. M. Rheinhardt aus Potsdam. In zahlreichen interessanten Diskussionen und Briefwechseln mit ihnen habe ich viel gelernt.

Ich danke Prof. U. Christensen, der diese Arbeit mitbetreut hat, sowie Prof. A. Tilgner für die Übernahme der Referate. 



\section{Lebenslauf}

Geburt: $\quad$ 5.3.1973 in Eschwege

Eltern: $\quad$ Norbert Schrinner

Ursula Schrinner,

geb. Oppermann

Staatsbürgerschaft: deutsch

Schulbildung: $\quad$ 8/1979 bis 7/1983 Alexander-von-Humboldt-Schule (Grundschule) 8/1983 bis 7/1989 Leuchtbergschule (Gymnasium)

8/1989 bis 6/1992 Oberstufengymnasium Eschwege

5/1992 Abitur

Zivildienst: $\quad$ 8/1992 bis $10 / 1993$

Studium: $\quad 10 / 1993$ bis 2/1999 Physikstudium an der

4/1996 Diplomvorprüfung

1/1998 bis 1/1999 Diplomarbeit:

Zur Nach-Hauptreihen Expansion

massereicher Sterne

2/1999

Diplomprüfung

$4 / 1999-12 / 2001$

Studium der Mathematik, Physik, Ev. Religion (Lehramt an Gymnasien)

$12 / 2001$

Erste Staatsprüfung

3/2002 bis 6/2005 Doktorarbeit am Max-Planck-Institut für Sonnensystemforschung in Katlenburg-Lindau 